\title{
Reactor-based neutrino oscillation experiments
}

\author{
Carlo Bemporad \\ Istituto Nazionale di Fisica Nucleare and University of Pisa, I-56010 Pisa, Italy
}

Giorgio Gratta

Physics Department, Stanford University, Stanford, California 94305

Petr Vogel

Physics Department, California Institute of Technology, Pasadena, California 91125

(Published 18 March 2002)

\begin{abstract}
The status of neutrino oscillation searches employing nuclear reactors as sources is reviewed. This technique, a direct continuation of the experiments that proved the existence of neutrinos, is today an essential tool in investigating the indications of oscillations found in studying neutrinos produced in the sun and in the earth's atmosphere. The low energy of the reactor $\bar{\nu}_{e}$ makes them ideal for exploring oscillations with small mass differences and relatively large mixing angles. In the last several years the determination of the reactor antineutrino flux and spectrum has reached a high degree of accuracy. Hence measurements of these quantities at a given distance $L$ can be readily compared with the expectation at $L=0$, thus testing the disappearance of $\bar{\nu}_{e}$. Two recent experiments, CHOOZ and PALO VERDE, with baselines of about $1 \mathrm{~km}$ and sensitive to the neutrino mass differences associated with the atmospheric neutrino anomaly have collected data and published results recently. An ambitious project with a baseline of more than $100 \mathrm{~km}$, KAMLAND, has now began to take data. This last reactor experiment will have a sensitivity sufficient to explore part of the oscillation phase space relevant to solar neutrino scenarios. It is the only envisioned experiment with a terrestrial source of neutrinos capable of addressing the solar neutrino puzzle.
\end{abstract}

I. Introduction

II. Physics Motivation for Modern Experiments
A. Experimental indications for neutrino oscillations
B. Reactor-based versus accelerator-based oscillation experiments

III. Reactor Neutrino Spectrum and Flux Determination
A. Antineutrino production
B. Fission-rate determination
C. From fission rates to the $\bar{\nu}_{e}$ spectra
D. From $\bar{\nu}_{e}$ to positrons
E. Accuracy of the flux and spectrum predictions
F. Other reactor neutrino experiments

IV. Experiments Motivated by the Atmospheric

Neutrino Anomaly
A. $\mathrm{CHOOZ}$
B. PALO VERDE
C. Backgrounds
D. Event reconstruction techniques
E. Results and systematics
F. Are smaller mixing angles within experimental reach?

V. Exploring the Solar $\nu$ Anomaly on Earth:

\section{KAMLAND}
A. Nuclear reactors in Japan
B. Detector design
C. Expected performance
D. Other physics with a very large low-energy $\bar{\nu}_{e}$ detector

VI. Conclusions

Acknowledgments

\section{INTRODUCTION}

Neutrinos have the distinction of being the first elementary particle whose existence was predicted by a theorist in order to explain seemingly unrelated phenomena. ${ }^{1}$ Pauli made this prediction in 1930 in his famous letter attempting to explain the continuous electron energy distribution in nuclear beta decay. It became immediately clear that neutrinos would be difficult to observe, because the corresponding cross sections are so tiny. But in a series of experiments from 1953 to 1959, Reines and Cowan $(1953,1959)$ were able to prove convincingly that electron antineutrinos from nuclear reactors are able to cause the inverse neutron beta decay, $\bar{\nu}_{e}+p \rightarrow e^{+}+n$, and hence that they are real particles. Shortly afterwards, in 1962, the separate identity of muon neutrinos, $\nu_{\mu}$, was demonstrated (Danby et al., 1962). Another decade later, in 1975, the $\tau$ lepton was discovered (Perl et al., 1975) and the observation of its decay properties implied the existence of a third neutrino, $\nu_{\tau}$, that was directly observed only very recently (Kodama et al., 2001). Precise measurements of the decay width of the $Z$ boson have shown that just three neutrino flavors $[2.994 \pm 0.012$ from the combined fit to

${ }^{1}$ For early developments in neutrino physics see, for example, Chap. 1 in Winter (1991). 
all Large Electron Positron Collider (LEP) data (Groom et al., 2000)] participate in weak interactions, at least for neutrinos with masses less than $M_{Z} / 2$.

Phenomenologically, it is obvious that neutrinos of each flavor are either massless or at least many orders of magnitude lighter than the corresponding charged leptons with which they form weak-interaction doublets. Based on these empirical facts, the standard model of electroweak interactions postulates that all neutrinos are massless and consequently have conserved helicity (which is the same as chirality in this case), and that the separate lepton numbers for electron, muon, and tau flavors are conserved. Challenging this postulate of the vanishing neutrino mass has recently become a central issue in many disciplines of fundamental science, including particle and nuclear physics, cosmology, and astrophysics. This review is devoted to one particular aspect of this broad effort.

Ironically, while our knowledge of intrinsic neutrino properties remains quite poor, these particles have been used as tools to understand other phenomena. The tradition of underground neutrino detectors began 30 years ago, when Davis and his collaborators were first able to detect neutrinos from the sun. (For a description of the history of solar neutrino research, see Bahcall, 1989.) Together with all the other experimental observations of solar neutrinos, this was, and still is, the only clear proof that the basic energy generation in stars is understood. The birth of neutrino astronomy can be associated with the observation of the neutrino burst from the supernova 1987A. Neutrino-induced reactions played an important role in establishing what is now known as the standard model of electroweak interactions when, in 1973, neutral currents were discovered via the observation of $\nu_{\mu}+e \rightarrow \nu_{\mu}+e$ scattering as well as neutralcurrent scattering of neutrinos on nucleons. Finally, neutrinos have been extensively used in deep-inelastic scattering experiments at CERN and FNAL, exploring the quark structure of nucleons.

The main problem in neutrino physics today is the question of whether neutrinos, like all charged fermions, have mass. ${ }^{2}$ Since direct kinematic tests of neutrino mass lack at present the required sensitivity, the recent hints of neutrino mass are indirect, based on the phenomenon of neutrino oscillations. If neutrinos are massive particles that behave analogously to quarks, the states with a definite mass (i.e., the "mass eigenstates" that propagate as plane waves in vacuum) are not necessarily the partners of the charged leptons that couple to the vector bosons $W^{ \pm}$in doublets (i.e., the weak or flavor eigenstates),

$$
\left(\begin{array}{c}
\nu_{e} \\
e^{-}
\end{array}\right), \quad\left(\begin{array}{c}
\nu_{\mu} \\
\mu^{-}
\end{array}\right), \quad\left(\begin{array}{c}
\nu_{\tau} \\
\tau^{-}
\end{array}\right)
$$

In such a case the weak eigenstates $\left|\nu_{l}\right\rangle$ will be linear superpositions of the mass eigenstates $\left|\nu_{i}\right\rangle$ :

\footnotetext{
${ }^{2}$ For an up-to-date discussion of neutrino masses and the relevant experiments, see Fisher, Kayser, and McFarland (1999) and Zuber (1998).
}

$$
\left|\nu_{l}\right\rangle=\sum_{i} U_{l, i}\left|\nu_{i}\right\rangle
$$

where the coefficients $U_{l, i}$ form the leptonic mixing matrix. If we assume that only three neutrinos can contribute to Eq. (2) above, then $U$ is a unitary $3 \times 3$ matrix. ${ }^{3}$

If Eq. (2) is valid, we encounter the phenomenon of neutrino oscillations, in which a neutrino that was initially in the weak eigenstate $l$ can be spontaneously transformed, at least in part, into another weakeigenstate neutrino of flavor $l^{\prime}$. [The idea of oscillations was discussed early on by Pontecorvo $(1958,1967)$ and by Maki, Nakagawa, and Sakata (1962).]

To see how that transformation happens, recall that the mass eigenstate $\left|\nu_{i}\right\rangle$ propagates according to the expression

$$
\left|\nu_{i}(t)\right\rangle=e^{-i\left(E_{i} t-p_{i} L\right)}\left|\nu_{i}(0)\right\rangle \simeq e^{-i\left(m_{i}^{2} / 2 E\right) L}\left|\nu_{i}(0)\right\rangle,
$$

where $L$ is the flight path, and the laboratory momenta and energies are assumed to be much larger than the neutrino rest masses $m_{i}$. Let us now consider the propagation of a neutrino that was created at $L=0$ as a weak eigenstate $\left|\nu_{l}\right\rangle$. At a distance $L$ this state is described by

$$
\begin{aligned}
\left|\nu_{l}(L)\right\rangle & \simeq \sum_{i} U_{l, i} e^{-i\left(m_{i}^{2} / 2 E\right) L}\left|\nu_{i}\right\rangle \\
& \simeq \sum_{l^{\prime}} \sum_{i} U_{l, i} e^{-i\left(m_{i}^{2} / 2 E\right) L} U_{l^{\prime}, i}^{*}\left|\nu_{l^{\prime}}\right\rangle .
\end{aligned}
$$

Here we used the inverse transformation to Eq. (2), i.e., from the mass eigenstates back to the weak eigenstates. This step must be taken, since the only way one can detect neutrinos is through their weak interactions. And in order to detect the neutrino flavor, we have to use charged-current weak interactions, characterized by the production of charged leptons $\left|l^{\prime}\right\rangle$.

Thus the neutrino of flavor $l$ acquired components corresponding to other flavors $l^{\prime}$. This is a purely quantum-mechanical effect, a consequence of the coherence in the superposition of states in Eq. (2). The probability that the "transition" $l \rightarrow l$ ' happens at $L$ is obviously

$$
\begin{aligned}
P\left(\nu_{l} \rightarrow \nu_{l^{\prime}}\right)= & \left|\sum_{i} U_{l, i} U_{l^{\prime}, i}^{*} e^{-i\left(m_{i}^{2} / 2 E\right) L}\right|^{2} \\
= & \sum_{i}\left|U_{l i} U_{l^{\prime} i}^{*}\right|^{2}+\Re \sum_{i} \sum_{j \neq i} \\
& \times U_{l i} U_{l^{\prime} i}^{*} U_{l^{*} j}^{*} U_{l^{\prime} j} e^{i\left(\left|m_{i}^{2}-m_{j}^{2}\right| L / 2 p\right) .}
\end{aligned}
$$

This is an oscillating function of the distance $L$. The oscillation length depends on the differences of the neutrino mass squares, $\left|m_{i}^{2}-m_{j}^{2}\right|$, and the oscillation amplitude depends on the mixing matrix $U$.

\footnotetext{
${ }^{3}$ Sometimes more than three mass eigenstates are considered. The additional neutrinos must be sterile or heavy, i.e., they must not participate in weak interactions so that the constraint from the invisible width of the $Z$ boson is obeyed.
} 
The necessary and sufficient conditions for the existence of neutrino oscillations are then (a) the nonvanishing value of at least one neutrino mass $m_{i}$ and (b) the nonvanishing value of at least one nondiagonal matrix element of the mixing matrix $U$. If these conditions are fulfilled, the individual lepton flavor numbers (electron, muon, and tau) are no longer conserved.

There is no fundamental theory that would allow us to deduce the parameters describing the mixing matrix $U$ and the mass differences $\Delta m_{i j}^{2}$. These unknown parameters must be determined empirically, by various neutrino oscillation experiments. Such analysis is often performed in a simplified way by assuming that only two neutrino flavors mix, e.g., $e$ and $\mu$. The mixing matrix $U$ then depends only on one mixing angle $\theta$, and the oscillation probability, Eq. (5), is also simplified:

$$
\begin{aligned}
& U=\left(\begin{array}{cc}
\cos \theta & \sin \theta \\
-\sin \theta & \cos \theta
\end{array}\right), \\
& P\left(\nu_{e} \rightarrow \nu_{\mu}, L\right)=\sin ^{2} 2 \theta \sin ^{2}\left(\Delta m^{2} L / 4 E\right) .
\end{aligned}
$$

Here $\Delta m^{2} \equiv m_{1}^{2}-m_{2}^{2}$, and we assume, as before, that the neutrinos are ultrarelativistic. The probability that $\nu_{e}$ remains $\nu_{e}$ is obviously

$$
P\left(\nu_{e} \rightarrow \nu_{e}, L\right)=1-P\left(\nu_{e} \rightarrow \nu_{\mu}, L\right) .
$$

In this two-flavor scenario the oscillation amplitude is $\sin ^{2} 2 \theta$, which vanishes if $\theta=0$ or $90^{\circ}$ and has a maximum if $\theta=45^{\circ}$. The oscillation length in meters is

$$
L_{o s c}=2 \pi \frac{2 E_{\nu}}{\Delta m^{2}}=\frac{2.48 E_{\nu}(\mathrm{MeV})}{\Delta m^{2}\left(\mathrm{eV}^{2}\right)} \mathrm{m} .
$$

To test for oscillations, one can perform either an appearance search in which one looks for a new neutrino flavor [i.e., the deviations of $P\left(\nu_{e} \rightarrow \nu_{\mu}, L\right)$ from zero], or a disappearance test, in which one looks for a change in the flux normalization [i.e., the deviation of $P\left(\nu_{e}\right.$ $\left.\rightarrow \nu_{e}, L\right)$ from unity]. In either case, tests performed at distance $L$ are only sensitive to the values of $\Delta m^{2}$ for which $L \geqslant O\left(L_{\text {osc }}\right)$. In other words, neutrino oscillations are observable only when $\Delta m^{2} L / E \sim O(1)$.

So far we have considered only propagation of neutrinos in a vacuum. When neutrinos propagate in matter, for example, in the solar interior, the oscillation pattern may be modified. This happens because electron neutrinos can forward scatter on electrons by charged-current interactions, and other neutrino flavors cannot. Under favorable circumstances a resonance enhancement of the oscillation amplitude, the so-called MikheyevSmirnov-Wolfenstein (MSW) effect (Wolfenstein, 1979, 1980; Mikheyev and Smirnov, 1986a, 1986b), can take place. Analogous matter-induced oscillations can distinguish the hypothetical sterile neutrinos, which have no weak interactions at all, from the $\nu_{\mu}$ or $\nu_{\tau}$ neutrinos, which interact with matter (electrons and quarks) by the neutral-current weak interaction. Neither of these effects is relevant for reactor neutrinos.

For completeness, it is worthwhile to mention here two other issues important in the study of intrinsic neu- trino properties. One of them is the behavior of neutrinos under charge conjugation. Unlike the charged leptons, which are Dirac particles with distinct antiparticles, neutrinos can be either Dirac or Majorana particles. In the latter case of truly neutral neutrinos, there is no distinction between the neutrinos and their antiparticles, and even the total lepton number is not conserved. In order to decide between these two possibilities, one has to look for processes that violate the total lepton number, such as the neutrinoless double beta decay. Other processes of this kind, like $\nu_{e} \rightarrow \bar{\nu}_{e}$ oscillations (e.g., in the present context the emission of $\nu_{e}$ from the nuclear reactor), are typically kinematically suppressed, and their observation is unlikely in the foreseeable future. The difference between the Dirac and Majorana neutrinos, while of fundamental importance, does not influence the results of the reactor oscillation searches described below.

The other issue worth mentioning is the possibility of $T$ or $C P$ violation in neutrino oscillations (Cabibbo, 1978; Barger, Whisnant, and Phillips, 1980). In order to establish violation of $T$ or $C P$, one would have to show that

$$
P\left(\nu_{\ell} \rightarrow \nu_{\ell}\right) \neq P\left(\bar{\nu}_{\ell} \rightarrow \bar{\nu}_{\ell}\right),
$$

i.e., that for example, the probability of $\nu_{\mu}$ oscillating into $\nu_{e}$ is different from the probability of $\bar{\nu}_{\mu}$ oscillating into $\bar{\nu}_{e}$.

For the usual case of three neutrino flavors, one can parametrize the lepton mixing matrix in terms of the three angles $\theta_{1}=\theta_{13}, \theta_{2}=\theta_{23}$, and $\theta_{3}=\theta_{12}$ and the $C P$-violating phase $\delta$ :

$$
\begin{aligned}
\left(\begin{array}{c}
\nu_{e} \\
\nu_{\mu} \\
\nu_{\tau}
\end{array}\right)= & \left(\begin{array}{ccc}
c_{1} c_{3} & c_{1} s_{3} & s_{1} e^{-i \delta} \\
-c_{2} s_{3}-s_{1} s_{2} c_{3} e^{i \delta} & c_{2} c_{3}-s_{1} s_{2} s_{3} e^{i \delta} & c_{1} s_{2} \\
s_{2} s_{3}-s_{1} c_{2} c_{3} e^{i \delta} & -s_{2} c_{3}-s_{1} c_{2} s_{3} e^{i \delta} & c_{1} c_{2}
\end{array}\right) \\
& \times\left(\begin{array}{c}
\nu_{1} \\
\nu_{2} \\
\nu_{3}
\end{array}\right)
\end{aligned}
$$

where $c_{1}=\cos \theta_{1}$ and $s_{1}=\sin \theta_{1}$, etc.

The magnitude of the $T$ or $C P$ violation is characterized by the differences

$$
\begin{aligned}
P(\bar{\mu} & \rightarrow \bar{e})-P(\mu \rightarrow e) \\
& =-[P(\bar{\mu} \rightarrow \bar{\tau})-P(\mu \rightarrow \tau)] \\
& =P(e \rightarrow \tau)-P(\bar{e} \rightarrow \bar{\tau}) \\
& =-4 c_{1}^{2} s_{1} c_{2} s_{2} c_{3} s_{3} \sin \delta\left[\sin \Delta_{12}+\sin \Delta_{23}+\sin \Delta_{31}\right],
\end{aligned}
$$

where $\Delta_{i j}=\left(m_{i}^{2}-m_{j}^{2}\right) \times L / 2 E$.

Thus the size of the effect is the same in all three channels, and $C P$ violation is observable only if all three masses are different (i.e., nondegenerate) and all three angles are nonvanishing. As will be shown below, reactor experiments constrain the angle $\theta_{1}$ (or $\theta_{13}$ ) to be small $\left(\sin ^{2} 2 \theta_{13} \leqslant 0.1\right)$. If that mixing angle vanishes exactly, no $C P$ violation is observable in the lepton sector, independently of the value of the $C P$-violating phase $\delta$. 
Further improvement of the sensitivity to $\sin ^{2} 2 \theta_{13}$ is, therefore, a matter of utmost importance.

\section{PHYSICS MOTIVATION FOR MODERN EXPERIMENTS}

Numerous searches for neutrino oscillations have been performed during the last two decades using nuclear reactors and particle accelerators as sources. Since most of them did not observe evidence for neutrino oscillations, their results are usually presented as an "exclusion plot," based on the simplified twoneutrino mixing scenario. That is, according to these plots, certain ranges of the parameters $\Delta m^{2}$ and $\sin ^{2} 2 \theta$ can be excluded from further consideration, as shown in Fig. 1. However, at the present time there are three groups of measurements that suggest the existence of neutrino oscillations. (And, at the same time, the parameter ranges suggested by them are not excluded.) Only these positive results will be briefly discussed here; other experiments are listed in the Review of Particle Physics (Groom et al., 2000).

\section{A. Experimental indications for neutrino oscillations}

The most prominent group of measurements that are commonly interpreted as evidence for neutrino oscillations are often referred to as the "atmospheric neutrino anomaly" (Kajita and Totsuka, 2001). Primary cosmic rays impinging on the nitrogen and oxygen nuclei at the top of the earth's atmosphere produce mostly pions, which subsequently decay via the chain $\pi^{-} \rightarrow \mu^{-} \bar{\nu}_{\mu}, \mu^{-}$ $\rightarrow e^{-} \bar{\nu}_{e} \nu_{\mu}$ (and the analogous chain with $\pi^{+}$, etc.). At sufficiently low energy, when such chains can fully develop, the resulting atmospheric neutrinos therefore are expected to follow the $\nu_{\mu}: \nu_{e}=2: 1$ ratio, which is essentially independent of the details of the complicated process that created them. In addition, in an underground detector, one can deduce the direction of the incoming high-energy neutrinos from the direction of the leptons $(e$ and $\mu$ ) created by the charged current interactions. Again, one is reasonably confident that this zenith-angle distribution can be accurately predicted. If the $\nu_{\mu}$ and/or $\nu_{e}$ neutrinos oscillate, one expects deviations from the 2:1 ratio mentioned above. Also, since the zenith angle is simply related to the neutrino path length, one expects deviations from the expected zenith-angle dependence of the lepton yield.

Both signatures of neutrino oscillations were in fact observed. The $\nu_{\mu} / \nu_{e}$ ratio is only about $60 \%$ of the expected value. This result has been confirmed in four detectors thus far. The anomalous zenith-angle dependence was first observed in Kamiokande, and has now been confirmed with much better statistical significance by Super-Kamiokande (Y. Fukuda, 1998; S. Fukuda 2000; Kajita and Totsuka, 2001). If these effects indeed signify neutrino oscillations (and we do not know of another viable explanation), then the corresponding mixing angle is large, $\sin ^{2} 2 \theta \approx 1$, and the value of the mass parameter $\Delta m^{2}$ is in the range $10^{-2}-10^{-3} \mathrm{eV}^{2}$.

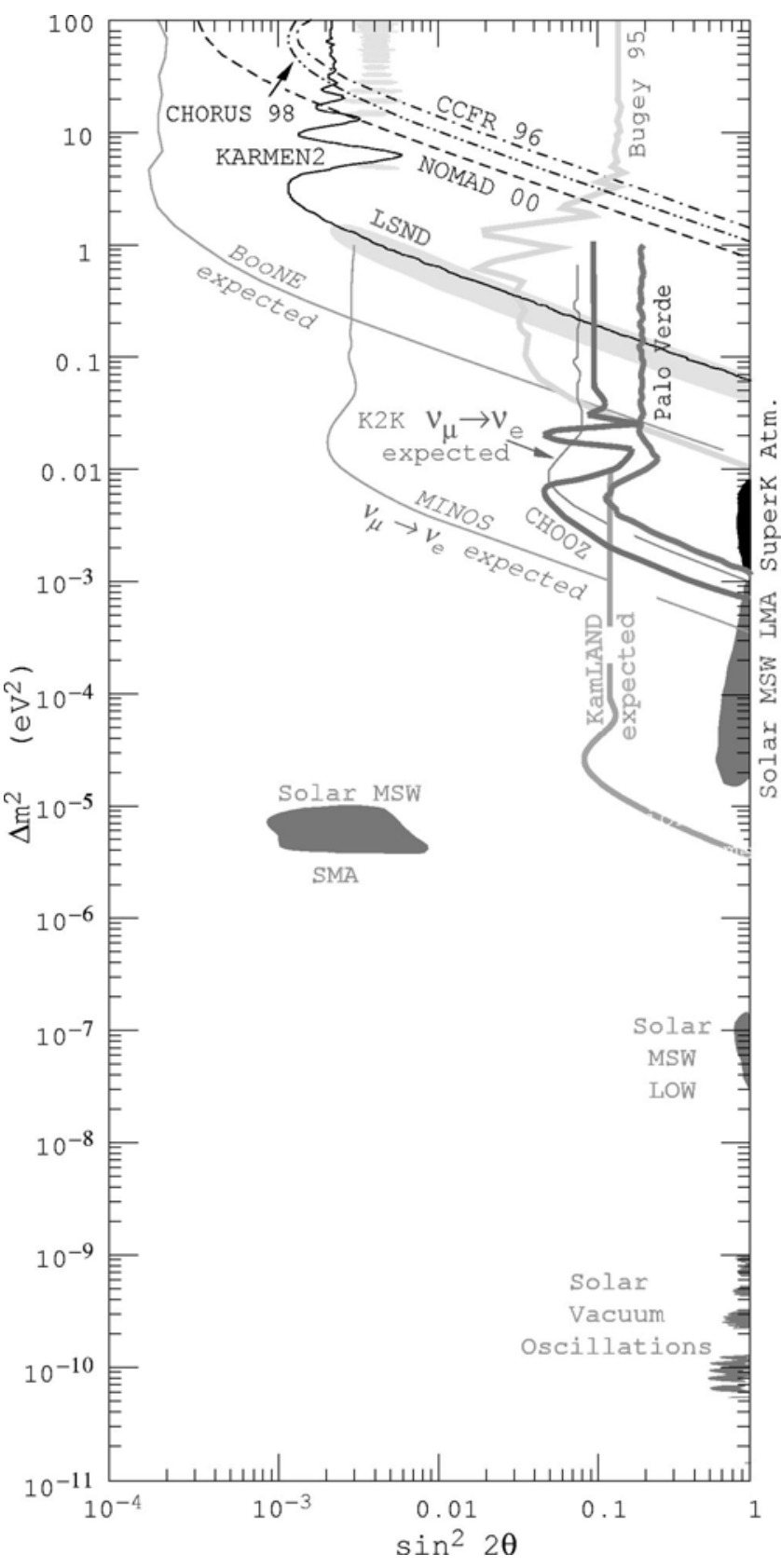

FIG. 1. Phase space for neutrino oscillations. The existing limits on $\nu_{e}-\nu_{\mu}$ are compared with current and future experiments and the regions obtained by interpreting the solar, atmospheric, and Liquid Scintillator Neutrino Detector (LSND) neutrino anomalies as due to oscillations (some of these effects are not necessarily $\nu_{e}-\nu_{\mu}$ oscillations.) The MikheyevSmirnov-Wolfenstein (MSW) mechanism is used in plotting some of the solar neutrino regions. The sensitivity of reactor experiments is the same for $\nu_{e}-\nu_{\tau}$ oscillations. Limits are at $90 \%$ C.L. The very recent Sudbury Neutrino Observatory (SNO) result has not been included in the analysis. SMA = small-mixing-angle solution; LMA = large-mixing-angle solution; LOW= low-mass solution.

While the preferred scenario at present involves $\nu_{\mu}$ $\rightarrow \nu_{\tau}$ oscillations, it is not clear that $\nu_{\mu}-\nu_{e}$ oscillations are fully excluded.

The second set of measurements that can be interpreted as evidence for neutrino oscillations deals with 
the "missing" solar neutrinos (see the review of earlier results in Kirsten, 2000; more recent results are presented by Ahmad, 2001 and S. Fukuda, 2001a, 2001b). The sun produces an intense flux of electron neutrinos as a by-product of the fusion reactions that generate solar power. It is believed that the solar structure is understood sufficiently well so that the flux and energy spectrum of the neutrinos can be confidently predicted. The solar neutrino fluxes have been measured in seven experiments so far. All of them report a deficit; i.e., the measured flux is less than the expected one. Moreover, the reduction depends on the neutrino energy, inferred experimentally from the thresholds of the individual detectors. The only viable explanation of the deficit appears to be neutrino oscillation ( $\nu_{e}$ disappearance). The hypothesis that solar $\nu_{e}$ indeed oscillate into "active" neutrinos that scatter on electrons via the neutralcurrent weak interaction is supported at the $3 \sigma$ level by combining the pure charged-current measurements of the Sudbury Neutrino Observatory (SNO; Ahmad, 2001) with the charged-current + neutral-current measurement of Super-Kamiokande (S. Fukuda, 2001a, 2001b).

In contrast to the attempts to explain the deficit by modification of the solar model, which are unsuccessful, all existing data can be simply and elegantly explained by invoking neutrino mass. In particular, the solution based on the MSW effect offers the most popular scenario. Treating the problem in the two-flavor framework explained above, one arrives at several isolated islands in the $\Delta m^{2}-\sin ^{2} 2 \theta$ plane. Two solutions correspond to $\Delta m^{2} \approx 10^{-5} \mathrm{eV}^{2}$. One of them, the "small mixing angle," has $\sin ^{2} 2 \theta \approx 10^{-2}$, while the other one, the "large mixing angle," has $\sin ^{2} 2 \theta \geqslant 0.5$. This latter solution, which currently gives the best fit to the data, spans an interval of $\Delta m^{2}$ extending up to $10^{-4} \mathrm{eV}^{2}$. The other possibilities have large mixing angles and $\Delta m^{2} \approx 10^{-7} \mathrm{eV}^{2}$ (the lowmass solution) or $\Delta m^{2} \approx 10^{-10} \mathrm{eV}^{2}$ (the vacuum solution).

Finally, the only indication of oscillations involving man-made neutrinos comes from the Liquid Scintillator Neutrino Detector (LSND) experiment, which finds evidence for the $\bar{\nu}_{\mu} \rightarrow \bar{\nu}_{\mathrm{e}}$ and, with more limited statistics, also for $\nu_{\mu} \rightarrow \nu_{\mathrm{e}}$ (Athanassopoulos et al., 1995, 1996, 1998; Aguilar et al., 2001). The former channel uses neutrinos from the pion and muon decay at rest, with energies less than $m_{\mu} / 2$. The latter channel uses neutrinos from the pion decay in flight which have somewhat higher energies. These are appearance experiments; the observed signal should be absent if neutrinos do not oscillate. The well-determined quantity is the oscillation probability, which has a value of about $3 \times 10^{-3}$. This result has not been independently confirmed. An analogous experiment that also uses neutrinos from pion and muon decay at rest, KARMEN (the KarlsruheRutherford Medium-Energy-Neutrino-Experiment; Armbruster et al., 1998; Eitel, 2000), found no evidence for the $\bar{\nu}_{\mu} \rightarrow \bar{\nu}_{\mathrm{e}}$ oscillations. However, the parameter space compatible with the LSND signal is not fully excluded by KARMEN.
As we can see from this brief discussion, the last decade has yielded a number of clues. With the exception of the LSND signal, they all came from measurements involving neutrinos produced by natural sources outside of our control. A number of new experiments have been performed or are in various stages of planning in order to investigate further these tantalizing effects. Reactor experiments play an all-important role in this quest, owing to their unique ability to investigate very small neutrino mass differences.

As in many other aspects of neutrino physics, there is a fundamental difference between the past reactor oscillation experiments ${ }^{4}$ and the more recent experiments with baselines of $1 \mathrm{~km}$ or more: experiments in this latter category are designed to further investigate, in a controlled environment with man-made neutrinos, particular regions of the oscillation parameter space where there are indications of oscillations from other experiments. Hence the results from the new generation of reactor $\bar{\nu}_{e}$ detectors directly impact our understanding of the neutrino mixing matrix.

\section{B. Reactor-based versus accelerator-based oscillation experiments}

Nuclear reactors produce isotropically $\bar{\nu}_{e}$ 's in the $\beta$-decay of neutron-rich fission fragments. All detectors optimized for oscillation searches take advantage of the relatively large cross section and specific signature of the inverse- $\beta$-decay reaction $p+\bar{\nu}_{e} \rightarrow n+e^{+}$. This cross section is shown in Fig. 2 as function of the neutrino energy along with the neutrino flux at the reactor and the resulting interaction rate in a particular detector configuration. We note here that the detection reaction has a threshold of about $1.8 \mathrm{MeV}$. Many of the merits and limitations of reactor-based experiments can be understood by observing that the energy of $\bar{\nu}_{e}$ is rather low, in the few-MeV range. It directly follows that reactorbased experiments can only be of the $\bar{\nu}_{e}$-disappearance type, since the neutrino "beam" does not have sufficient energy to produce muons (or taus), and the neutralcurrent reactions of the "oscillated" $\bar{\nu}_{\mu}$ or $\bar{\nu}_{\tau}$ have small cross sections and are indistinguishable from the many backgrounds present. This first limitation makes reactorbased experiments well suited only for investigating relatively large mixing angles. In practice experiments have reported mixing sensitivities around $10 \%$ at large $\Delta m^{2}$ (although the proposal for a very ambitious experiment with sensitivity better than $2 \%$ at a particular $\Delta m^{2}$ will be discussed later). The second limitation of reactorbased oscillation searches derives from the fact that the only known method of collimating neutrino beams employs the Lorentz boost of the parent particles from

\footnotetext{
${ }^{4}$ For a general discussion of short-baseline reactor experiments see Boehm and Vogel (1992) and Boehm (2001); for the individual experiments see Kwon et al. (1981), Zacek et al. (1986), Achkar (1992), Vidyakin et al. (1994), Achkar et al. (1995, 1996), and Alfonin et al. (1998).
} 


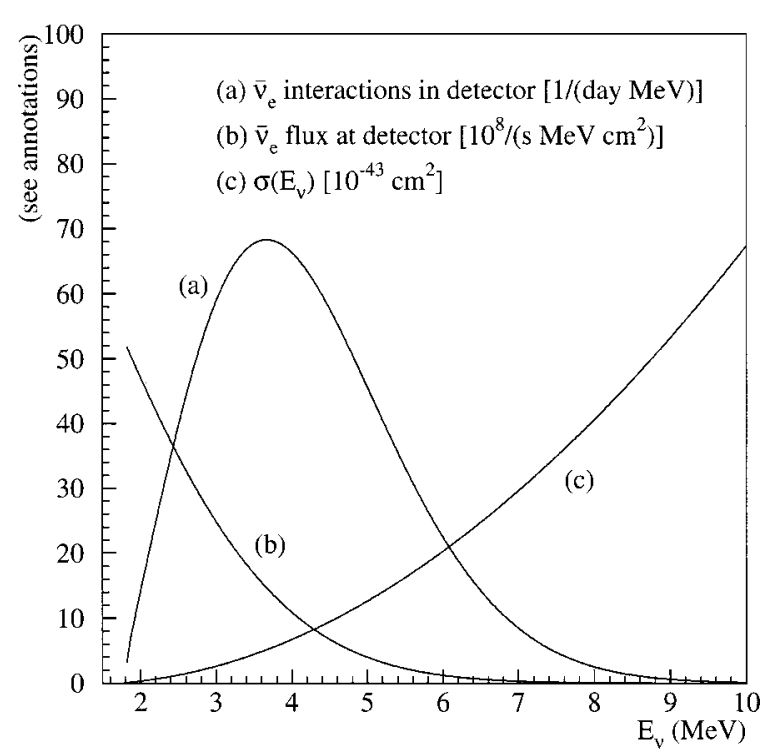

FIG. 2. Reactor $\bar{\nu}_{e}$ flux, inverse-beta-decay cross section, and $\bar{\nu}_{e}$ interaction spectrum at a detector based on such a reaction: (a) and (b) refer to a 12-ton fiducial mass detector located 0.8 $\mathrm{km}$ from $12-\mathrm{GW}_{\text {th }}$ power reactor.

whose decay the neutrinos are produced. For this reason low-energy neutrinos are generally produced over large solid angles, while high-energy ones may come in relatively narrow beams. Obviously a reactor emits $\bar{\nu}_{e}$ 's in a completely isotropic way, and this, together with the modest interaction cross sections available at low energy, makes the specific signal rates rather low. At the same time, however, low-energy neutrinos provide us with a unique opportunity to probe the lowest regions of $\Delta m^{2}$ that are otherwise beyond the reach of acceleratorbased searches. Some of these tradeoffs are well illustrated by Fig. 3, where the $\Delta m^{2}$ sensitivity is shown, together with the necessary baseline, versus the reactor power and detector fiducial mass.

Oscillation searches using reactors as sources are particularly important today, since several of the indications for neutrino oscillations shown in Fig. 1 point to regions of the parameter space at small $\Delta m^{2}$ and nearly full mixing. Hence two reactor-based experiments, $\mathrm{CHOOZ}$ and PALO VERDE, were performed to investigate the phenomenon of atmospheric neutrinos as $\bar{\nu}_{e} \rightarrow \bar{\nu}_{x}$ oscillations. Such experiments, described in detail below, had baselines of about $1 \mathrm{~km}$ and fiducial masses of the order of 10 tons. In comparison, the much more complex accelerator-based Main Injector Neutrino Oscillation Search (MINOS) between FNAL and the Soudan mine (Wojcicki, 2001a) and analogous projects between CERN and Gran Sasso, OPERA and ICARUS (Oscillation Project with Emulsion-Tracking Apparatus and Imaging Cosmic and Rare Underground Signal utilizing a liquid argon tracker; for a brief description, see, for example, Wojcicki, 2001b), will access similar $\Delta m^{2}$ values with $\mathrm{GeV}$-energy neutrinos and a baseline of the order of $1000 \mathrm{~km}$. However, the 5400-ton MINOS detector and its analogs at Gran Sasso will also be able to investigate oscillation channels not including $\bar{\nu}_{e}$ and

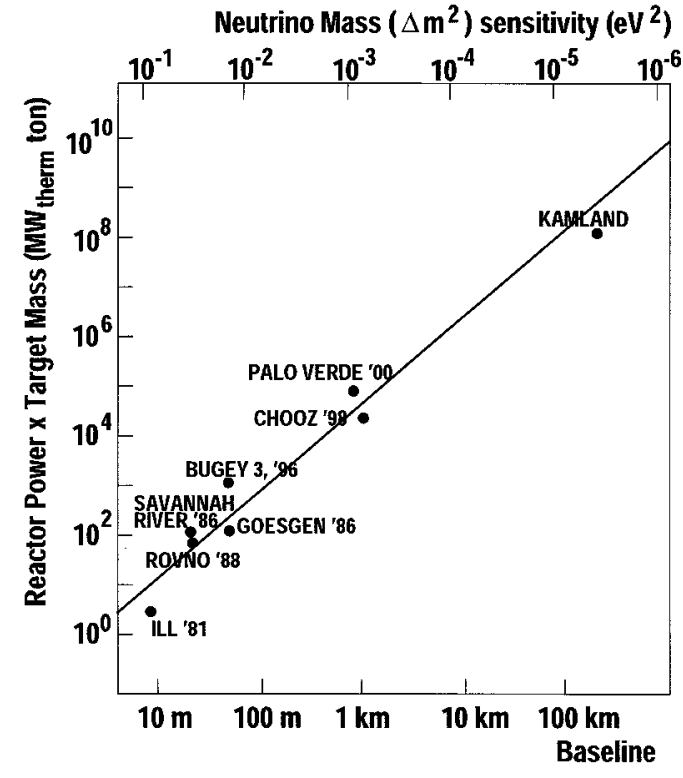

FIG. 3. Neutrino $\Delta m^{2}$ sensitivity as a function of total reactor power and detector fiducial mass for detection based on the inverse- $\beta$ reaction discussed in the text. The baseline scales with the $\Delta m^{2}$ sensitivity sought according to Eq. (8). The fiducial mass $\times$ power necessary for the experiment grows with the square of the baseline. The past experiments are labeled by the name of the reactor complex used. The approximate year of the experiment is also indicated to show that the increased baseline and $\Delta m^{2}$ sensitivity more or less followed the chronological order.

reach a mixing parameter sensitivity substantially better than $1 \%$.

The reactor-based KAMLAND experiment, with a baseline larger than $100 \mathrm{~km}$, will offer a unique opportunity of testing, with man-made neutrinos, the largemixing-angle MSW solution of the solar neutrino puzzle. In this case the restriction to $\bar{\nu}_{e} \rightarrow \bar{\nu}_{x}$ oscillations does not limit the interest of the experiment (since solar neutrinos certainly do involve $\nu_{e}$ ), while its $\Delta m^{2}$ sensitivity is well beyond what can be practically achieved by accelerators (in comparison, similar $\Delta m^{2}$ sensitivity could be achieved in an accelerator-based experiment with baselines of order $10^{5} \mathrm{~km}$, larger than the diameter of the earth).

Of course, the relatively lower energy of neutrinos from reactors pushes the optimization of reactor-based experiments to concentrate on the reduction and rejection of backgrounds from natural radioactivity, which is hardly an issue in accelerator-based detectors. In this respect the correlated signature of the inverse- $\beta$ process, the detection of the $e^{+}$and neutron, plays a very important role.

While in the case of neutrinos produced by accelerators the experimenter has full control over the status of the beam, the flux of $\bar{\nu}_{e}$ 's cannot be changed at will in commercial power nuclear reactors. However, in practice, typical reactor optimization requires a refueling shutdown every 12 to 24 months. Such shutdowns usually last about a month, providing a convenient flux modulation that can be used to validate background 
subtraction methods. As explained in detail later, even in the case of KAMLAND, which observes the neutrinos from about 70 reactor cores, a substantial flux modulation is provided by the coincidence of scheduled refueling outages in the spring and fall, when electricity demand is lowest.

Finally, we remark here that the fully isotropic flux produced by nuclear reactors eliminates the problems related to beam pointing that are present in experiments using accelerators. While the pointing accuracy required in these experiments is well within the present technology, a foolproof cross check of the beam detector alignment is certainly not trivial to obtain.

In conclusion, reactor-based and accelerator-based experiments offer complementary approaches to the quest for neutrino oscillations. It is likely that only combined efforts on these two fronts, together with other studies such as the search for neutrinoless double $\beta$ decay, will allow us to elucidate the problem of mixing in the lepton sector.

\section{REACTOR NEUTRINO SPECTRUM AND FLUX DETERMINATION}

Since reactor-based oscillation experiments are of the disappearance type, the accurate determination of the $\bar{\nu}_{e}$ spectrum and its absolute normalization are essential ingredients of the measurements. We note here that for oscillation parameters well within the experimental sensitivity, the evidence for oscillations would manifest itself as a deficit of events accompanied by a distortion of the energy spectrum, as shown by the example in Fig. 4. However, as the true value of the oscillation parameters moves closer to the sensitivity boundary of the experiment, the spectral shape loses power, and the accuracy of the measurements essentially relies on the total event count and, hence, the knowledge of the absolute reactor flux. This last scenario also corresponds to the more usual case, in which no oscillations are observed and an upper limit is set, as well as to the case of large $\Delta m^{2}$, in which the spectrum distortions are washed out.

While in this section we shall concern ourselves mainly with a priori reactor $\bar{\nu}_{e}$ yield determinations, multiple-baseline measurements are possible and have been performed in the past at the Goesgen (Zacek et al., 1986) and Bugey (Achkar et al., 1995, 1996) reactors. Indeed, such measurements helped instill confidence in the reactor yield estimates, and, although they were not the main goal of CHOOZ and PALO VERDE, they have recently been proposed (Mikaelyan, 2000) for a more accurate determination of the mixing angle $\theta_{13}$ for the atmospheric neutrino region. $\mathrm{CHOOZ}$ could take advantage of the $\approx 115 \mathrm{~m}$ distance between the reactors to derive weaker exclusion limits, which were however less affected by systematics.

\section{A. Antineutrino production}

The determination of the $\bar{\nu}_{e}$ yield proceeds schematically in three steps. First, the thermal power of each

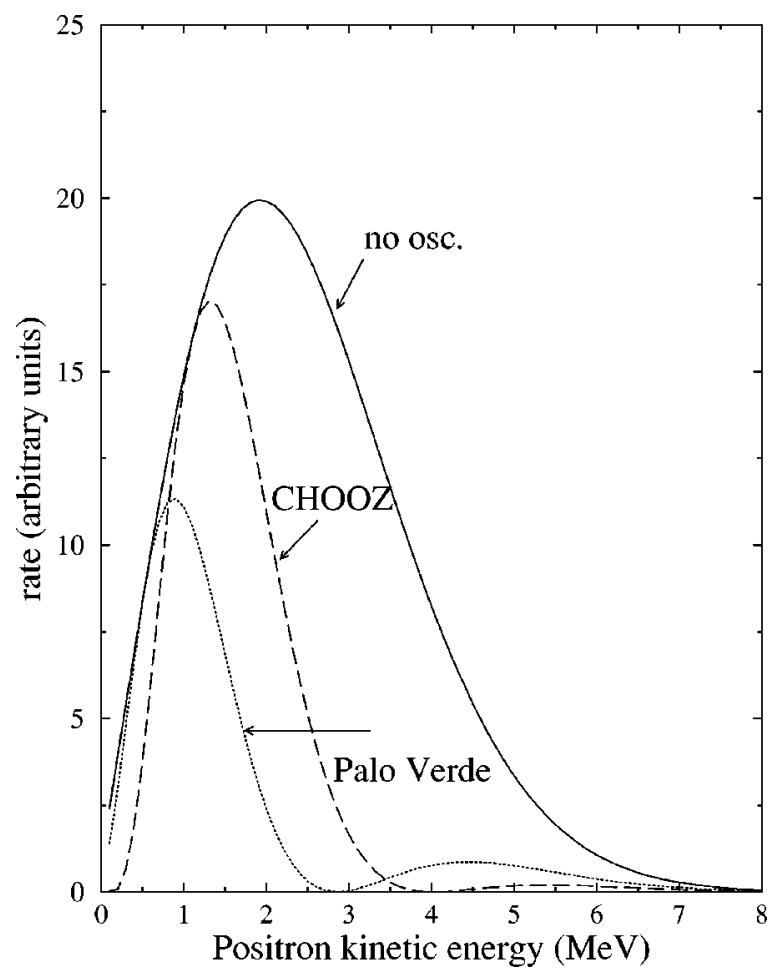

FIG. 4. Expected positron energy spectra: solid line, no oscillations; dashed line, oscillations with parameters $\Delta m^{2}=7.2$ $\times 10^{-3} \mathrm{eV}^{2}$ and $\sin ^{2} 2 \theta=1$ at the CHOOZ $(L \simeq 1 \mathrm{~km})$ experiment; dotted line, the same oscillations at the PALO VERDE ( $L \simeq 0.8 \mathrm{~km}$ ) experiment. Adapted from Harrison, Perkins, and Scott (1996).

reactor core is measured accurately and essentially continuously. Based on such measurements, and starting from the initial fuel composition, the burnup state can be computed as a function of time. Small corrections due to other reactor parameters that modify the criticality of the core are also introduced at this time. Reactor simulation codes are often used at this stage and produce an accurate instantaneous fission rate for each of the relevant isotopes through the fuel cycle. In the second step the neutrino spectrum is derived from the fission rate. Finally, the neutrino spectrum emitted by the reactors must be converted into an estimate of the experimental observable, the positron spectrum in the detector. Each of these steps will be explained in a separate subsection.

Typical modern commercial light-water reactors have thermal powers of the order of $3 \mathrm{GW}_{t h}$. This figure applies to both pressurized-water reactor and the less common boiling-water reactor designs. In both cases the fuel is enriched to $2-5 \%$ in ${ }^{235} \mathrm{U}$. Since on average each fission produces $\sim 200 \mathrm{MeV}$ and $\sim 6 \bar{\nu}_{e}$, we conclude that the typical yield is $\sim 6 \times 10^{20} \bar{\nu}_{e}$ core $^{-1} \mathrm{~s}^{-1}$ (of course part of this flux will be below the detection threshold; see Fig. 2).

It is easy to understand why $\sim 6 \bar{\nu}_{e}$ are produced per fission. Take, as an example, the most common ${ }^{235} \mathrm{U}$ fission, which produces two unequal fragments and typically two new neutrons that sustain the chain reaction, 


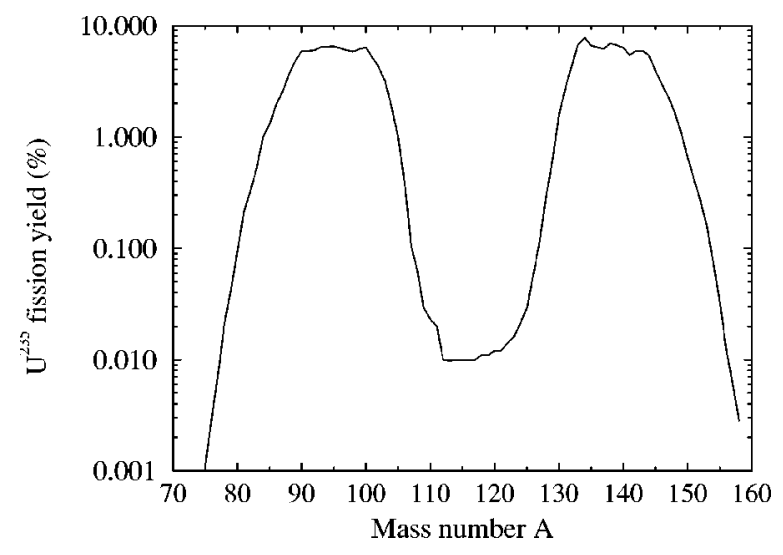

FIG. 5. Yields (in \%) for ${ }^{235} \mathrm{U}$ thermal neutron fission (normalized to $200 \%$ for the two fragments).

$$
{ }^{235} \mathrm{U}+n \rightarrow X_{1}+X_{2}+2 n .
$$

The mass distribution of the fragments (so-called fission yields) is shown in Fig. 5. The lighter fragments have, on average, $A \simeq 94$ and the heavier ones have $A$ $\simeq 140$. The stable nucleus with $A=94$ is ${ }_{40} \mathrm{Zr}^{94}$ and the stable $A=140$ nucleus is ${ }_{58} \mathrm{Ce}^{140}$. These two nuclei together have 98 protons and 136 neutrons, while the initial fragments, as seen from the equation above, have 92 protons and 142 neutrons. To reach stability, therefore, on average 6 neutrons bound in the fragments have to undergo $\beta$ decay, emitting the required $6 \bar{\nu}_{e}$.

While the total number of $\bar{\nu}_{e}$ 's is easy to estimate and can be accurately determined given the known fission yields, their energy spectrum, which is of primary interest for the oscillation searches discussed here, requires more care. In particular, the commonly used neutrino detection reaction, the inverse neutron $\beta$ decay, has a threshold of $\sim 1.8 \mathrm{MeV}$. Only about $1.5 \bar{\nu}_{e}$ /fission (i.e., $\sim 25 \%$ ) of the total are above that threshold and hence can be detected.

The existence of the $1.8-\mathrm{MeV}$ threshold in the detection process $\bar{\nu}_{e}+p \rightarrow n+e^{+}$automatically ensures that only $\bar{\nu}_{e}$ 's from large- $Q$-valued, and hence short-half-life, $\beta$ decays are detected. Thus the observed $\bar{\nu}_{e}$ signal tracks closely in time the power excursions in the reactor. This is of some practical importance, as large quantities of spent fuel are usually stored on site by reactor operators. There is no need to track the inventory of spent fuel and to worry about the $\beta$ decays of the neutron-activated reactor materials, which typically have a low $Q$ value and therefore long-half-life products. In practice, after a few hours from reactor turn on/off, the detectable $\bar{\nu}_{e}$ flux can be considered saturated.

\section{B. Fission-rate determination}

The four isotopes whose fission is the source of virtually all the reactor power are ${ }^{235} \mathrm{U},{ }^{238} \mathrm{U},{ }^{239} \mathrm{Pu}$, and ${ }^{241} \mathrm{Pu}$. The fission rates deriving from their evolution during a typical fuel cycle in one of the Palo Verde reactors is shown in Fig. 6, as calculated by a core simula-

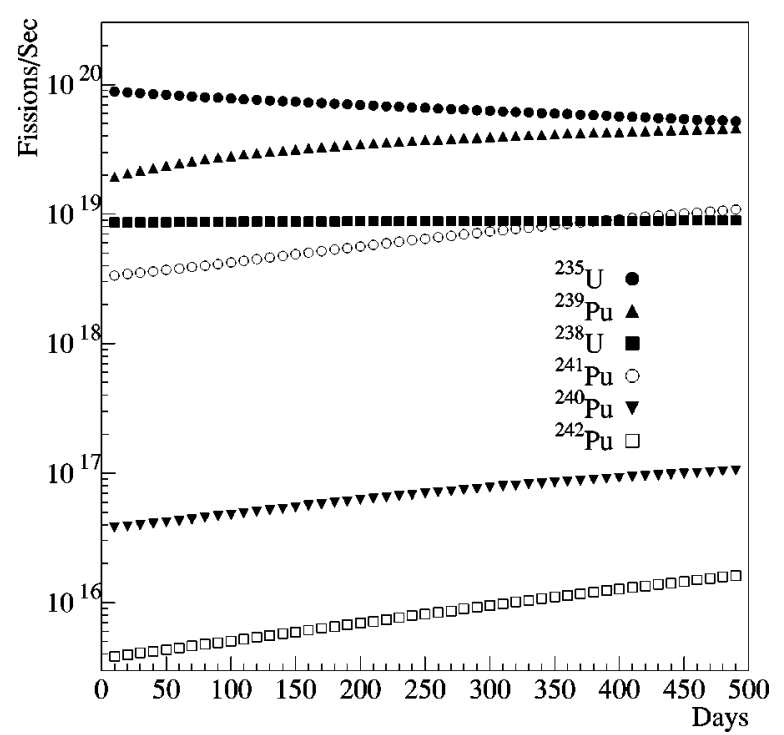

FIG. 6. Time evolution of fission rates for each of the six most important isotopes in one of the Palo Verde reactor cores. The horizontal scale covers a full fuel cycle, at the end of which about $1 / 3$ of the core is replaced with fresh fuel. Only the four most important isotopes are normally used to predict $\bar{\nu}_{e}$ yields.

tion program (Miller, 2000). For comparison, we also show the evolution of ${ }^{240} \mathrm{Pu}$ and ${ }^{242} \mathrm{Pu}$, which give the next-to-leading contributions. The contribution of these isotopes is of order $0.1 \%$ or less and will not be considered further.

Each isotope produces a unique neutrino spectrum through the decay of its fission fragments and their daughters, so plutonium breeding results in a small but noticeable change in the emitted neutrino spectrum.

Two types of uncertainties can be attributed to the isotope compositions described in Fig. 6: errors deriving from uncertainties in the initial fuel composition and in the measurement of the plant parameters that are used as input to the simulation, and errors due to imperfect core and neutronics modeling by the simulation program itself. The errors intrinsic to the simulation are known to contribute substantially less than $1 \%$ to the neutrino yield from tests in which fuel is sampled and analyzed for isotopic composition at the end of a fuel cycle.

The correlation between the $\bar{\nu}_{e}$ yield and the plant parameters used as input to the simulation is shown in Fig. 7. Apart from the obvious correlation with the thermal power, other parameters enter the simulation because they affect the criticality by altering the neutron transport in the core (generally by the water density and boron absorber concentration). We see that for the parameter with largest correlation besides power, the water temperature in the cold legs, an error of $10 \%$ produces an uncertainty of only $0.15 \%$ in the $\bar{\nu}_{e}$ yield. Of course the inlet temperature is known to much better than $10 \%$.

Economic and safety reasons provide plant operators with an incentive for measuring the thermal power of the reactors accurately. Indeed, usually more than one method is used and the results are compared to understand the size of the uncertainties. Calorimetric methods 

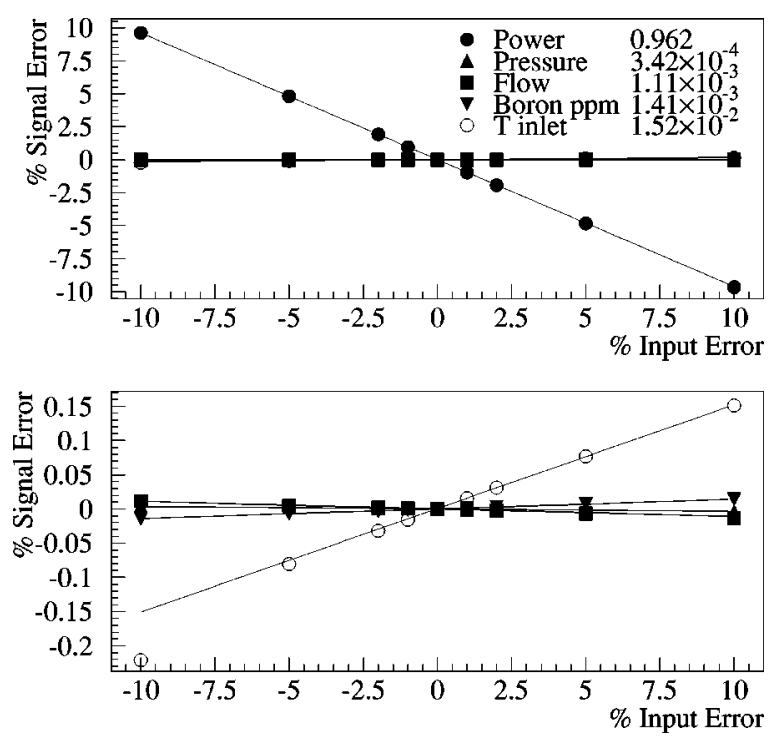

FIG. 7. Correlation between $\bar{\nu}_{e}$ yield and the five most important inputs to the core simulation for a pressurized-water reactor. The numbers in the key are the slopes of the fitted lines. In the top panel four of the five lines are superimposed in a trend close to slope zero. These four lines are separated in the expanded scale in the bottom panel. Note that a variation of even $10 \%$ in any of the parameters but power has little effect on the output of the simulation.

(simultaneous measurement of temperature and flow rate of the water outlet in the secondary cooling loop) give the smallest error $(\sim 0.6-0.7 \%$ at $\mathrm{CHOOZ}$ and PALO VERDE) and are used as a primary power estimate.

\section{From fission rates to the $\bar{\nu}_{e}$ spectra}

The instantaneous fission rates of the four isotopes ${ }^{235} \mathrm{U},{ }^{238} \mathrm{U},{ }^{239} \mathrm{Pu}$, and ${ }^{241} \mathrm{Pu}$ found above are then used as an input for the evaluation of the $\bar{\nu}_{e}$ spectrum. For all but ${ }^{238} \mathrm{U}$, careful measurements of the $\beta$ spectrum from fission by thermal neutrons were performed (Schreckenbach et al., 1985; Hahn et al., 1989). These are converted to neutrino spectra as explained below. However, Schreckenbach et al. and Hahn et al. do not include ${ }^{238} \mathrm{U}$, which undergoes only fast neutron fission and hence was not accessible to such measurements.

There are, at present, several methods available for evaluating the $\bar{\nu}_{e}$ spectrum. For the $\bar{\nu}_{e}$ 's associated with ${ }^{235} \mathrm{U},{ }^{239} \mathrm{Pu}$, and ${ }^{241} \mathrm{Pu}$, which undergo thermal neutron fission, it is customary to use a hybrid method, based on the conversion of the measured electron spectra associated with the thermal neutron-induced fission into the $\bar{\nu}_{e}$ spectra. Clearly, the electron and $\bar{\nu}_{e}$ originate from the same $\beta$ decay and share the available end-point energy. For a single branch the conversion is therefore trivial. However, in general there are many branches and many nuclei with different charges. For electron and antineutrino energies well above the electron mass, the two spectra are quite similar (Schreckenbach et al., 1985):

$$
\frac{d N}{d E_{e}} \simeq \frac{d N}{d E_{\bar{\nu}_{e}}},
$$

where $E_{e}=\sqrt{p_{e}^{2}+m_{e}^{2}}$ is the full electron energy, and the proportionality constant deviates from unity by at most $5 \%$. Naturally, one would like to convert the spectra more accurately.

Formally, the conversion can be performed exactly as follows. Let $n(E, Z)$, assumed to be a continuous function, describe the distribution of end points $E$ and nuclear charges $Z$. The electron spectrum is then

$$
\begin{aligned}
Y\left(E_{e}\right)= & \int_{E_{e}}^{\infty} d E n(E, Z) k(E, Z) p_{e} \\
& \times E_{e}\left(E-E_{e}\right)^{2} F\left(E_{e}, Z\right),
\end{aligned}
$$

where $k(E, Z)$ is the spectrum normalization constant, $p_{e}$ is the electron momentum, and $F\left(E_{e}, Z\right)$ is the Fermi function describing the Coulomb effect on the emitted electron. Provided that the electron spectrum $Y\left(E_{e}\right)$ is measured, the end-point distribution can be determined,

$$
n(E, Z)=-\frac{1}{2 k(E, Z)} \frac{d^{3}}{d E^{3}}\left(\frac{Y(E)}{p E F(E, Z)}\right) .
$$

Once the distribution $n(E, Z)$ is known, the $\bar{\nu}_{e}$ spectrum is readily calculated by the integral analogous to Eq. (14). In Davis et al. (1979) and Vogel et al. (1981) it was shown that such a conversion procedure depends only very weakly on the value of $Z$. In practice, an empirical relation $\bar{Z}(E)$ between the average $\bar{Z}$ and the electron energy has been used (Schreckenbach et al., 1985): $\bar{Z}$ $=49.5-0.7 E-0.09 E^{2}$, with $E$ in $\mathrm{MeV}$.

When using the measured electron spectra, the above expressions, involving third derivatives, are obviously impractical. Instead, the integral in Eq. (14) is replaced by a finite sum of 30 hypothetical beta-decay branches with branching ratios $b_{i}$ and equidistant end points $E_{0}^{i}$,

$$
\begin{aligned}
Y\left(E_{e}\right)= & \sum_{i} b_{i} k\left(E_{0}^{i}, \bar{Z}\right) \delta\left(E_{e}, E_{0}^{i}\right) p_{e} \\
& \times E_{e}\left(E_{0}^{i}-E_{e}\right)^{2} F\left(E_{e}, \bar{Z}\right),
\end{aligned}
$$

where $\delta\left(E_{e}, E_{0}^{i}\right)$ describes the small outer radiative corrections. One can now begin with the largest value of $E_{0}^{i}$ (only one branch) and determine the corresponding branching ratio $b_{i}$ using the electrons of energies between that $E_{0}^{i}$ and the next smaller one, and continue in this fashion step by step until the smallest $E_{0}^{i}$ is reached. Possible variations in the number and distribution of the end points $E_{0}^{i}$ affect the resulting $\bar{\nu}_{e}$ spectrum by no more than 1\% (see Schreckenbach et al., 1985). Having determined the set $b_{i}, E_{0}^{i}$, it is straightforward to obtain

$$
\begin{aligned}
Y\left(E_{\bar{\nu}_{e}}\right)= & \sum_{i} b_{i} k\left(E_{0}^{i}, \bar{Z}\right) E_{\bar{\nu}_{e}}^{2}\left(E_{0}-E_{\bar{\nu}_{e}}\right) \\
& \times\left[\left(E_{0}-E_{\bar{\nu}_{e}}\right)^{2}-m_{e}^{2}\right]^{1 / 2} F\left(E_{0}-E_{\bar{\nu}_{e}}, \bar{Z}\right),
\end{aligned}
$$

where the irrelevant radiative corrections were omitted. This is the procedure used in deriving the neutrino spec- 
tra associated with fission of ${ }^{235} \mathrm{U},{ }^{239} \mathrm{Pu}$, and ${ }^{241} \mathrm{Pu}$, which account for about $90 \%$ of the reactor $\bar{\nu}_{e}$ 's.

The $\bar{\nu}_{e}$ spectra in Davis et al. (1979), Vogel et al. (1981), Klapdor and Metzinger (1982a, 1982b), Schreckenbach et al. (1985), and Hahn et al. (1989), are given as tables. A somewhat less accurate, but easier-toimplement, analytical approximation is given by Vogel and Engel (1989). [The fit error to the total rate is about $1.2 \%$ for ${ }^{235} \mathrm{U}$ and only about $0.3 \%$ for ${ }^{239} \mathrm{Pu}$ and ${ }^{241} \mathrm{Pu}$ (Miller, 2000).]

For the $\bar{\nu}_{e}$ associated with ${ }^{238} \mathrm{U}$ fission, one has to use the straightforward summation of the spectra of the $\bar{\nu}_{e}$ 's from all individual $\beta^{-}$decays. Thus

$$
\frac{d N}{d E_{\bar{\nu}}}=\sum_{n} Y_{n}(Z, A, t) \sum_{i} b_{n, i}\left(E_{0}^{i}\right) P_{\bar{\nu}}\left(E_{\bar{\nu}}, E_{0}^{i}, Z\right),
$$

where $Y_{n}(Z, A, t)$ is the number of $\beta$ decays per unit time of the fragment $Z, A$ after the fissioning material has been exposed to neutrons for a time $t$, and the label $n$ characterizes each fragment. For $t$ larger than the $\beta$-decay lifetime of the fragment $Z, A$, the quantity $Y_{n}$ converges toward the cumulative fission yield and becomes independent of $t$. Naturally, each fission fuel is characterized by a different set of yields $Y_{n}$.

The quantities $b_{n, i}\left(E_{0}^{i}\right)$ in Eq. (18) are the branching ratios for the $i$ th branch with the maximal electron energy (end-point energy) $E_{0}^{i}$. The branching ratios are normalized to unity.

Finally, the function $P_{\bar{\nu}}\left(E_{\bar{\nu}}, E_{0}^{i}, Z\right)$ is the normalized spectrum shape. It is usually assumed, here and in the conversion method explained above, that all relevant $\beta$ decays have the allowed shape,

$$
\begin{aligned}
P_{\bar{\nu}}\left(E_{\bar{\nu}}, E_{0}, Z\right)= & k\left(E_{0}, Z\right) E_{\bar{\nu}}^{2}\left(E_{0}-E_{\bar{\nu}}\right) \\
& \times\left[\left(E_{0}-E_{\bar{\nu}}\right)^{2}-m_{e}^{2}\right]^{1 / 2} \\
& \times F\left(E_{0}-E_{\bar{\nu}}, Z\right) .
\end{aligned}
$$

Equation (19) is a very good approximation in practice and causes a totally negligible error.

The weakness of this method is the incomplete information on the end-point distribution and branching ratios of some fission fragments, in particular those with very short lifetimes and high decay energies. These "unknown" decays contribute as much as $25 \%$ of the $\bar{\nu}_{e}$ 's at energies above $4 \mathrm{MeV}$. In practice, nuclear models are used to supplement the missing data. Examples of calculations based on this method are those of Davis et al. (1979), Vogel et al. (1981), Klapdor and Metzinger (1982a, 1982b), and Tengblad et al. (1989). An example of an extension to lower $\bar{\nu}_{e}$ energies, where the neutron activation of the reactor materials plays a role, is given by Kopeikin, Mikaelyan, and Sinev (1997).

While the summation method played an important role in early oscillation searches, at present it is needed only for the description of the $\bar{\nu}_{e}$ from ${ }^{238} \mathrm{U}$ fission as pointed out above. That component contributes only about $11 \%$ to the neutrino signal. We show below that the error associated with the summation method is less than $10 \%$ and hence it contributes less than $1 \%$ to the overall uncertainty.

The ultimate check of the accuracy of the prediction outlined above consists in comparing the results in terms of $\bar{\nu}_{e}$ energy spectrum with the measurements performed in short-baseline reactor oscillation experiments.

\section{From $\bar{\nu}_{e}$ to positrons}

Since in all reactor experiments one measures the positron spectra, and not the $\bar{\nu}_{e}$ spectra directly, one has to understand quantitatively how these are related. In other words, one has to know the cross section of the "detector" reaction $\bar{\nu}_{e}+p \rightarrow e^{+}+n$.

The total cross section for this reaction, neglecting terms of order $E_{\nu} / M$, is given by the standard formula

$$
\begin{aligned}
\sigma_{\text {tot }}^{(0)} & =\sigma_{0}\left(f^{2}+3 g^{2}\right) E_{e}^{(0)} p_{e}^{(0)} \\
& =0.0952\left(\frac{E_{e}^{(0)} p_{e}^{(0)}}{1 \mathrm{MeV}^{2}}\right) \times 10^{-42} \mathrm{~cm}^{2},
\end{aligned}
$$

where $E_{e}^{(0)}=E_{\nu}-\left(M_{n}-M_{p}\right)$ is the positron energy when the (small) neutron recoil is neglected, and $p_{e}^{(0)}$ is the corresponding momentum. The vector and axialvector coupling constants are $f=1, g=1.26$, and

$$
\sigma_{0}=\frac{G_{F}^{2} \cos ^{2} \theta_{C}}{\pi}\left(1+\Delta_{\text {inner }}^{R}\right)
$$

where the energy-independent inner radiative corrections are $\Delta_{\text {inner }}^{R} \simeq 0.024$.

The cross section can be expressed in terms of the neutron lifetime and the phase-space factor $f_{p . s .}^{R}$ $=1.7152$ (Wilkinson, 1982) as

$$
\sigma_{\text {tot }}^{(0)}=\frac{2 \pi^{2} / m_{e}^{5}}{f_{p . s .}^{R} \tau_{n}} E_{e}^{(0)} p_{e}^{(0)} .
$$

In this way, the cross section is tied directly to the neutron lifetime $\tau_{n}$, known to $0.2 \%$ (Groom et al., 2000); no knowledge of $G_{F}, f / g$, or the Cabibbo angle $\theta_{C}$ is in fact needed.

The (small) energy-dependent outer radiative corrections to $\sigma_{\text {tot }}$ are given by Vogel (1984a) and Fayans (1985). The corrections to the cross section of order $E_{\nu} / M$, which are not negligible even for the reactor energies, and the angular distribution of the positrons are described by Vogel and Beacom (1999). The exact threshold of the reaction is

$$
E_{\nu}^{t h r}=\frac{\left(M_{n}+m_{e}\right)^{2}-M_{p}^{2}}{2 M_{p}}=1.806 \mathrm{MeV}
$$

instead of just $M_{n}+m_{e}-M_{p}=1.804 \mathrm{MeV}$ when the recoil is neglected.

Using the results of Vogel and Beacom (1999), one can evaluate the total cross section as well as the quantity $\left\langle\cos \theta_{e}\right\rangle$ that characterizes the positron angular distribution essentially exactly. These quantities are shown in Fig. 8. The high-energy extension of the total and differential cross section has already been discussed in 


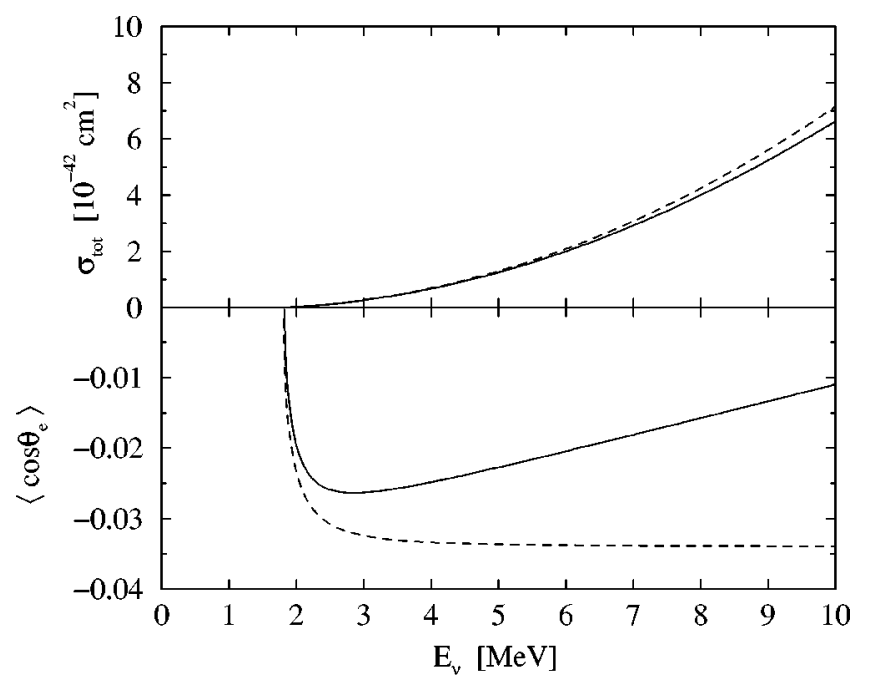

FIG. 8. $\bar{\nu}_{e}+p \rightarrow n+e^{+}$reaction: Upper panel, total cross section; bottom panel, $\langle\cos \theta\rangle$ as a function of the antineutrino energy; solid lines, $\mathcal{O}\left(1 / M_{n}\right)$ result; dashed lines, $\mathcal{O}(1)$ result [Eq. (20)].

the classic paper by Llewellyn-Smith (1972). Near the threshold, however, that treatment must be modified as shown by Vogel and Beacom (1999).

The positron angular distribution, characterized by $\left\langle\cos \theta_{e}\right\rangle$, is rarely accessible. It is of interest, however, to consider the angular distribution of the recoil neutrons that are also detected. Since in the laboratory system the proton is at rest, the neutron is initially emitted at a forward angle restricted by

$$
\cos \left(\theta_{n}\right)_{\max }=\frac{\sqrt{2 E_{\nu} \Delta-\left(\Delta^{2}-m_{e}^{2}\right)}}{E_{\nu}},
$$

where $\Delta=M_{n}-M_{p} \sim 1.3 \mathrm{MeV}$. The average $\left\langle\cos \left(\theta_{n}\right)\right\rangle$ is considerably closer to unity than $\cos \left(\theta_{n}\right)_{\max }$ (Vogel and Beacom, 1999).

It is often possible to localize the points where the positron was created and where the neutron was captured, and even though the neutron undergoes many elastic scatterings before capture, its final position maintains some memory of its original direction. Simulations indicate that the typical displacement of the two vertices is $\langle x\rangle \sim 1.5 \mathrm{~cm}$ in the organic scintillator. In fact, in previous reactor experiments (Zacek, 1984; Zacek et al., 1986; Achkar, 1992; Achkar et al., 1995) the neutron displacement was clearly observed, in the Goesgen experiment in particular, at $\simeq 10 \sigma$ level. The same effect was also observed at PALO VERDE. Moreover, the singlevessel $\mathrm{CHOOZ}$ experiment was able to measure the average neutron-positron separation and to base on it a determination of the $\bar{\nu}_{e}$ incoming direction with an uncertainty of $\simeq 8^{\circ}$ (Apollonio et al., 2000).

Given a reliable simulation of the neutron transport, this asymmetry allows, albeit with large errors, a direct measurement of the detector background. In the case of $\bar{\nu}_{e}$ detection from a future supernova, this technique may provide, as shown by Apollonio et al. (2000), a crude but useful determination of the direction to the $\bar{\nu}_{e}$ source, i.e., of the star position.

\section{E. Accuracy of the flux and spectrum predictions}

Once the cross section and $\bar{\nu}_{e}$ spectra are known, the corresponding positron yield is easily evaluated. In reactor experiments, the neutron recoil is quite small (10-50 $\mathrm{keV}$ ), and thus the positron energy is simply related to the incoming $\bar{\nu}_{e}$ energy,

$$
E_{\nu} \simeq\left(E_{e}+\Delta\right)\left[1+\frac{E_{e}}{M_{p}}\right]+\frac{\Delta^{2}-m_{e}^{2}}{M_{p}},
$$

where, as before, $\Delta=M_{n}-M_{p}$, and $\cos \theta_{e}=0$ is used as a good approximation of the average $\left\langle\cos \theta_{e}\right\rangle$. We note here that possible detector efficiency dependence on the positron energy requires special care.

In order to check the accuracy of the prediction, one has to compare the results, in terms of the $\bar{\nu}_{e}$ energy spectrum and total flux normalization, with the measurements performed in short-baseline reactor oscillation experiments. Since such experiments have not reported the observation of oscillations, we can assume that their measurements represent the direct determination of the reactor spectrum at production. There are four factors needed for the evaluation of the expected positron yield and spectrum: the distance to each core, the number of target protons, the cross section for $\bar{\nu}_{e}+p \rightarrow n+e^{+}$, and of course the quantity one wants to test, the $\bar{\nu}_{e}$ spectrum at the source.

The distance to the reactors is trivially obtained with negligible uncertainty. Determining the number of protons in the target requires knowledge of the chemical composition and mass of both scintillator and other possible detector materials in which $\bar{\nu}_{e}$ 's can be captured and recorded with finite efficiency. Typically errors smaller than $1 \%$ are achievable for this parameter. The cross section was discussed in the previous subsection, and its uncertainty is also less than $1 \%$. Finally, the $\bar{\nu}_{e}$ spectrum is the object of the test.

The $\bar{\nu}_{e}$ flux normalization alone was tested by the Bugey 4 experiment (Declais et al., 1994), in which the total neutron yield was measured with high statistics. The experimental accuracy of neutrino flux was $1.4 \%$, compared with an accuracy of $2.7 \%$ of the expected flux. The Bugey 4 experimental result therefore agrees with expectations based on the known neutron lifetime at the $3 \%$ level. It is interesting to note that the same experiment was performed previously, with the same detector, at the Rovno reactor (Kuvshinnikov, 1990).

The validity of the tests performed at short distances from a reactor is reinforced by the fact that some of the experiments such as Goesgen (Zacek et al., 1986) or Bugey (Achkar et al., 1995, 1996) observed no difference between spectra measured at different baselines. This is shown for Goesgen in Fig. 9. Moreover, the relatively recent Bugey 3 measurements were performed at a distance of 15,40 , and $95 \mathrm{~m}$ from the core and recorded very high statistics (some $1.5 \times 10^{5} \bar{\nu}_{e}$ events at the shorter distances). 


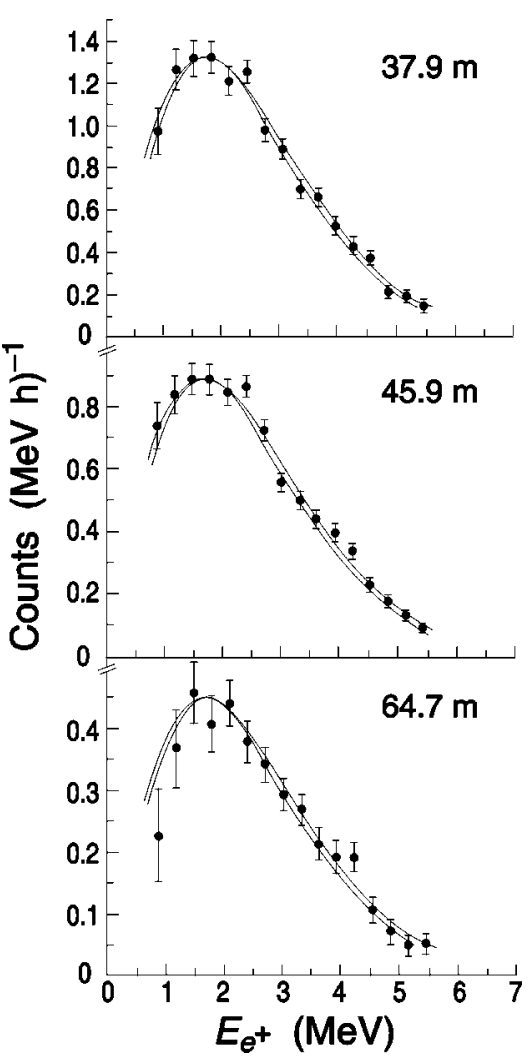

FIG. 9. Positron spectrum observed by the Goesgen experiment for three different baselines (Zacek, 1984): upper lines, fits to the data; lower lines, predictions obtained as described in the text, using the measurements of Schreckenbach et al., 1985, and Hahn et al., 1989, for ${ }^{235} \mathrm{U},{ }^{239} \mathrm{Pu}$, and ${ }^{241} \mathrm{Pu}$ and theoretical calculations for ${ }^{238} \mathrm{U}$.

The good agreement between the Bugey 3 data and the nonoscillation predictions is shown in Fig. 10. In panel (a) the prediction is generated purely from theory. Of more practical importance is panel (b), where the prediction is derived from $\beta$ spectra (except for ${ }^{238} \mathrm{U}$, for
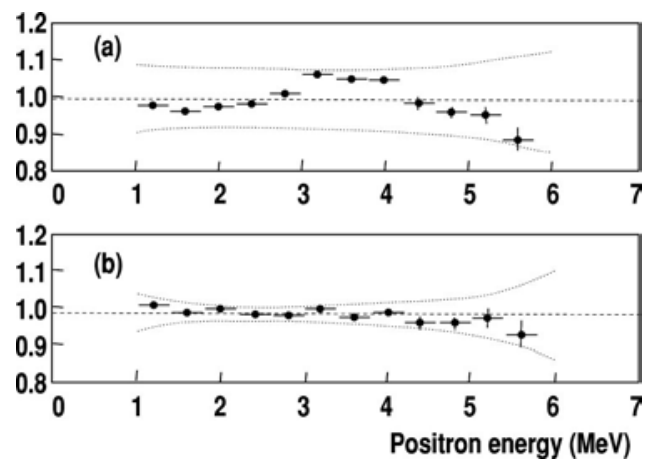

FIG. 10. Ratio between Bugey 3 measurements and different predictions: (a) comparison of the measurements to the a priori calculations of Klapdor and Metzinger (1982a, 1982b); (b) comparison of Bugey 3 data to the prediction obtained using the $\beta$ spectra measurements of Schreckenbach et al. (1985) and Hahn et al. (1989), and the calculation mentioned for ${ }^{238} \mathrm{U}$. The dotted envelopes are estimates of the overall systematics. Adapted from Achkar et al. (1996).

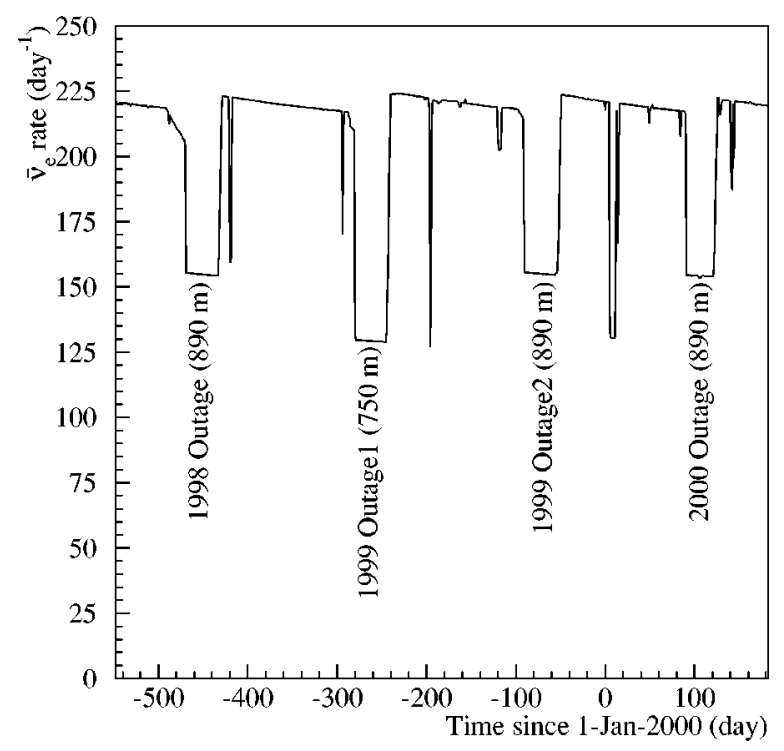

FIG. 11. Expected number of $\bar{\nu}_{e}$ interactions in the PALO VERDE detector during the $\sim 2$ years of data taking of the experiment. Note that one reactor is closer to the experiment while the other two are equidistant; this explains the different excursion for one of the refuelings. The steady decline in $\bar{\nu}_{e}$ interactions during the cycle is the effect of fuel burnup.

which theory is used). In this case a fit to a horizontal line gives a level of 0.99 with $\chi^{2} / \mathrm{DOF}$ (degree of freedom) $=9.2 / 11$.

For reference we give in Fig. 11 the time evolution of the $\bar{\nu}_{e}$ interaction rate expected in the PALO VERDE detector, calculated as described above from the plant data. This time evolution is typical of a plant with more than one reactor. Refueling outages (about 1 month long each) give different rate excursions due to the different distances between the reactors and the detector. Short accidental reactor trips are also visible along with the steady rate decline through the reactor cycle due to fuel burnup.

In Fig. 12 the measured effect of the changing reactor fuel composition is shown for the $\mathrm{CHOOZ}$ experiment. At the same time, the figure demonstrates how small that effect really is. We plot in Fig. $12 \sigma_{f} / E_{f}$, since the number of events $n_{\nu}$ at a given time and fuel composition is

$$
n_{\nu}=\frac{1}{4 \pi R^{2}} \frac{W_{t h}}{E_{f}} N_{p} \varepsilon \sigma_{f},
$$

where $R$ is the distance, $N_{p}$ is the number of protons, $\varepsilon$ is efficiency for the event detection, $\sigma_{f}$ is the effective cross section per fission, and $E_{f}$ is the average energy per fission; $\sigma_{f}$ and $E_{f}$ are both sensitive to the burnup.

In conclusion, the $\bar{\nu}_{e}$ spectra and their absolute normalization are known to about $2 \%$ accuracy. Obviously, the reactors as $\bar{\nu}_{e}$ sources are perfectly isotropic. The differences between various reactors and the time changes due to the fuel burnup are small and well understood. They do not cause additional uncertainty. Thus, for the reactor neutrino oscillation searches at a few percent accuracy, no short-distance "monitor" detectors are needed. One can simply compare the mea- 


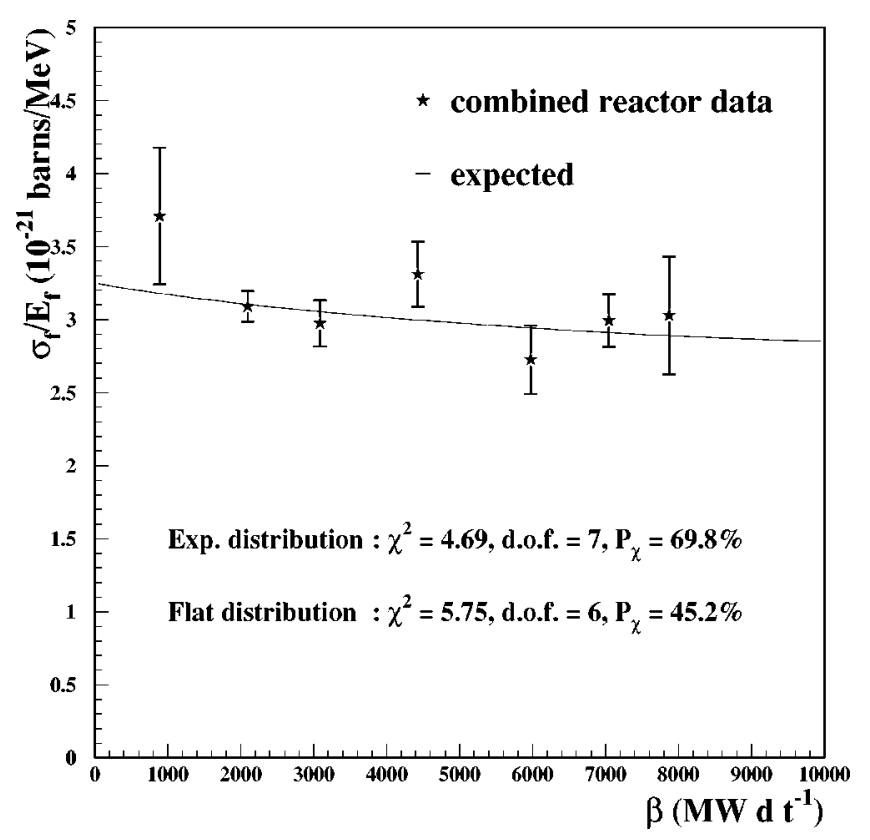

FIG. 12. The measured $\bar{\nu}_{e}$ event rate at $\mathrm{CHOOZ}$, expressed as the cross section per fission divided by the energy per fission (see text for explanation), as a function of the reactor burnup $\beta$ (in units of $\mathrm{MW} \times$ days per ton), i.e., the accumulated reactor power per unit mass of fuel. The fits to the expected rate, which includes the reactor burnup, and to the flat rate, where the burnup is neglected, are also shown. Adapted from Nicolò (1999).

sured positron spectra at the distance $L$ to the expectation at the source $(L=0)$.

\section{F. Other reactor neutrino experiments}

We shall only briefly mention here other experiments with reactor $\bar{\nu}_{e}$ 's that are of importance as a potential source of information about intrinsic neutrino properties. Besides protons, two targets have been extensively studied: deuterons and electrons.

Reactor $\bar{\nu}_{e}$ 's can cause deuteron disintegration by two reaction channels, the charged current

$$
\bar{\nu}_{e}+d \rightarrow e^{+}+n+n,
$$

with a threshold of $4.03 \mathrm{MeV}\left(M_{n}+m_{e}-M_{p}+B\right)$, where $B$ is the deuteron binding energy, and the neutral current

$$
\bar{\nu}_{e}+d \rightarrow \bar{\nu}_{e}+p+n,
$$

with a lower threshold of $B=2.23 \mathrm{MeV}$. The cross sections of these reactions, averaged over the reactor $\bar{\nu}_{e}$ spectrum, are $\sim 1.1 \times 10^{-44} \mathrm{~cm}^{2} /$ fission for the charged current, and $\sim 3.1 \times 10^{-44} \mathrm{~cm}^{2} /$ fission for the neutral current (see, for example, Davis et al., 1979 and Vogel et al., 1981). These are more than an order of magnitude smaller than for the reaction on the proton target. (The cross section is expressed "per fission" because the fission rate is more directly related to the reactor power than the $\bar{\nu}_{e}$ flux.)
The study of the $\bar{\nu}_{e}+d$ reactions was pioneered by Reines and collaborators (Pasierb et al., 1979; Reines et al., 1980), who observed the corresponding two- or one-neutron captures. From the point of view of neutrino oscillations, the ratio of the charged- and neutralcurrent rates is potentially useful, since the charged current is flavor sensitive and the neutral current is not (the same idea is being pursued in the case of solar neutrinos by the SNO Collaboration). More recent experiments (Riley et al., 1999; Kozlov et al., 2000) show no indication of oscillations, as expected given the short distance from the reactor.

The other reaction observed with reactor $\bar{\nu}_{e}$ 's is the scattering on electrons,

$$
\bar{\nu}_{e}+e \rightarrow \bar{\nu}_{e}+e,
$$

where the spectrum of the recoil electrons (originally assumed to be at rest in the laboratory, since the electron momentum associated with the atomic binding is usually negligible) is observed. Obviously, the reaction signature, just the recoiling electron, is quite difficult to distinguish from background caused by radioactivity, making the observation of the $\bar{\nu}_{e}-e$ scattering very challenging.

The cross section for $\bar{\nu}_{e}-e$ scattering consists of the well-understood weak-interaction part and a so far unobserved incoherent electromagnetic part:

$$
\begin{aligned}
\frac{d \sigma}{d T}= & \frac{G_{F}^{2} m_{e}}{2 \pi}\left[(f+g)^{2}+(f-g)^{2} \times\left(1-\frac{T}{E_{\nu}}\right)^{2}\right. \\
& \left.+\left(g^{2}-f^{2}\right) \frac{m_{e} T}{E_{\nu}^{2}}\right]+\frac{\pi \alpha^{2} \mu_{\nu}^{2}}{m_{e}^{2}} \frac{1-T / E_{\nu}}{T}
\end{aligned}
$$

where $T$ is the kinetic energy of the recoiling electron. The terms proportional to $G_{F}^{2}$, weak scattering, represent the sum of coherent (interfering) contributions from charged and neutral currents, while the second part, proportional to $\alpha^{2}$, can be described as due to a finite neutrino magnetic moment $\mu_{\nu}$. Only massive neutrinos can have magnetic moments, and hence the study of this reaction, and the possible determination of $\mu_{\nu}^{2}$, is of great interest.

Again, pioneering results were obtained by Reines and collaborators (Reines, Gurr, and Sobel, 1976). More recently, limits on $\mu_{\nu}^{2}$ with reactor neutrinos, albeit with a rather poor signal-to-noise ratio, were obtained by Derbin et al. (1993). At present a slightly more stringent direct limit of $\mu_{\nu} \leqslant 1.6 \times 10^{-10} \mu_{\mathrm{B}}$ comes not from the reactor neutrinos, but from the analysis (Beacom and Vogel, 1999) of the shape of the Super-Kamiokande solar neutrino data (Y. Fukuda et al., 1999). A new effort to improve the sensitivity to $\mu_{\nu}^{2}$ is currently underway (Amsler et al., 1997) at the Bugey reactor Magnetic Moment of the Neutrino (MUNU) experiment.

Finally, the search for neutrino decay also provides information about neutrino mass and mixing. Indeed, if neutrinos have a mass and are mixed, the heavier ones can and will decay into the lighter ones. Heavy neutri- 
nos, if they exist, must decay sufficiently rapidly not to overclose the universe (see, for example, Raffelt, 1995).

Two decay modes in particular have been studied with reactor neutrinos, the radiative decay

$$
\nu_{2} \rightarrow \nu_{1}+\gamma,
$$

with no threshold (except that $m_{2}>m_{1}$ ), and the decay

$$
\nu_{2} \rightarrow \nu_{1}+e^{+}+e^{-},
$$

possible only if $m_{\nu_{2}}-m_{\nu_{1}}>2 m_{e}$. The Review of Particle Physics (Groom et al., 2000) lists the most recent results of the searches for these and other decay modes. In particular, various limits (often stringent) based on astrophysics are listed there.

In the standard model, radiative decay has a very long lifetime, much longer than the age of the universe. But alternative models, such as those with right-handed weak interactions, often allow faster decays. Hence a program of searching for neutrino decay has been a part of the study of reactor neutrinos. The first results were obtained again by Reines and collaborators (Reines, Sobel, and Gurr, 1974). More restrictive limits were reached by Vogel (1984b) and by Oberauer and von Feilitzsch (1987). Bouchez et al. (1988) searched in particular for photons in the visible energy range, giving them high sensitivity to small $\Delta m / m$ values. A more recent search was performed by Schoenert et al., 1996.

The heavy-neutrino decay mode into the $e^{+}+e^{-}$pair plus a light neutrino will dominate the total decay rate above its threshold, since it results from a simple $W$ exchange; there is no Glashow-Iliopoulos-Maiani suppression as in the radiative mode. To observe this decay mode with reactor neutrinos, it would be necessary that heavy neutrinos exist and that they couple to electrons. Thus the decay rate would contain the mixing amplitude $\left|U_{e 2}\right|^{4}$. For a given mass $m_{2}$ of the heavy neutrino, one could then find a limit on the mixing amplitude $\left|U_{e 2}\right|^{2}$. The most stringent limits obtained to data with reactor neutrinos were those found Hagner et al. (1995).

\section{EXPERIMENTS MOTIVATED BY THE ATMOSPHERIC NEUTRINO ANOMALY}

Two experiments have been built with the specific purpose of testing the hypothesis that neutrino oscillations occur with the parameters found by the atmospheric neutrino measurements. Both experiments are now completed. All CHOOZ data were published by Apollonio et al. (1998, 1999), and Nicolò (1999). Data-taking at PALO VERDE was completed in the summer of 2000, and the results were published by Boehm et al. (2000a, 2000b, 2001) and Miller (2000).

As can be seen from Fig. 3 , in order to access $\Delta m^{2}$ $\approx 10^{-3} \mathrm{eV}^{2}$ with reactor neutrinos, a baseline of order 1 $\mathrm{km}$ and a mass in excess of a few tons are needed. Indeed, the backgrounds from cosmic radiation and natural radioactivity are a major consideration in the design of such large, low-energy detectors, and different background situations led the two groups to rather different designs.
The CHOOZ detector was built in a preexisting underground cavity under $\mathrm{a} \simeq 100$ - $\mathrm{m}$ rock overburden $\simeq 300$ mwe (meter of water equivalent). This substantial cosmic radiation shielding allowed the use of a homogeneous detector in which inverse- $\beta$ events were tagged as a double (delayed) coincidence between the $e^{+}$ (prompt) signal and the $n$ (delayed) signal. Such a simple event signature can be identified with high efficiency, so that a 5-ton active mass was sufficient for the experiment.

The PALO VERDE detector, on the other hand, was located in an underground bunker excavated for the purpose. Economic considerations limited the overburden to $12 \mathrm{~m}(\simeq 32 \mathrm{mwe})$, sufficient only to eliminate the hadronic component of the cosmic radiation and reduce the muon flux by a factor of five. The rather large remaining muon flux produced a substantial number of secondary neutrons, so that a segmented detector was needed to take full advantage of the triple coincidence given by the $e^{+}$ionization and subsequent $\gamma$ 's from annihilation. This more elaborate topological signature reduced the detector efficiency and pushed the fiducial mass to 12 tons. Both detectors were built with materials selected for low radioactivity and included a passive $\gamma$ and neutron shield and an active cosmic-ray veto counter.

While the three-reactor plant of PALO VERDE has a thermal power of $11.6 \mathrm{GW}_{t h}$, the two reactors of $\mathrm{CHOOZ}$ produce $8.5 \mathrm{GW}_{t h}$. More importantly, the $\mathrm{CHOOZ}$ reactors were commissioned only after the start of data taking at the experiment. This gave the collaboration the unusual opportunity to observe the backgrounds at reactor-off for a substantial period and during the slow ramp-up of power. The more stable operating conditions of PALO VERDE, with the periodic $\sim 2 / 3$ power excursions shown in Fig. 11, made the task of understanding and subtracting backgrounds more challenging. At the same time the methods developed in this context will have universal value in future experiments that will likely encounter similar steady-state conditions.

Both detectors used liquid scintillators loaded with $0.1 \%$ natural gadolinium, which has a high thermal neutron capture cross section and releases a large amount of energy in the capture. In this way the neutron capture time is reduced to $\sim 27 \mu$ s from $\sim 170 \mu$ s for the unloaded scintillator, proportionally reducing the uncorrelated background. Furthermore, Gd deexcitation after the capture releases an $8-\mathrm{MeV} \gamma$ cascade, whose summed energy gives a robust event tag well above natural radioactivity. In contrast, neutron capture on protons results in only a single $2.2-\mathrm{MeV} \gamma$. While the $\mathrm{Gd}$ loading offers obvious advantages in suppressing backgrounds, it is not easy to achieve in the stable and sufficiently transparent form needed for a large detector. Both groups invested substantial resources in scintillator development. In Fig. 13 we show initial attenuation length data for the PALO VERDE scintillator, which was a cocktail of $60 \%$ mineral oil, $36 \%$ pseudocumene (1,2,4-trimethylbenzene), and 4\% alcohol (used to keep 

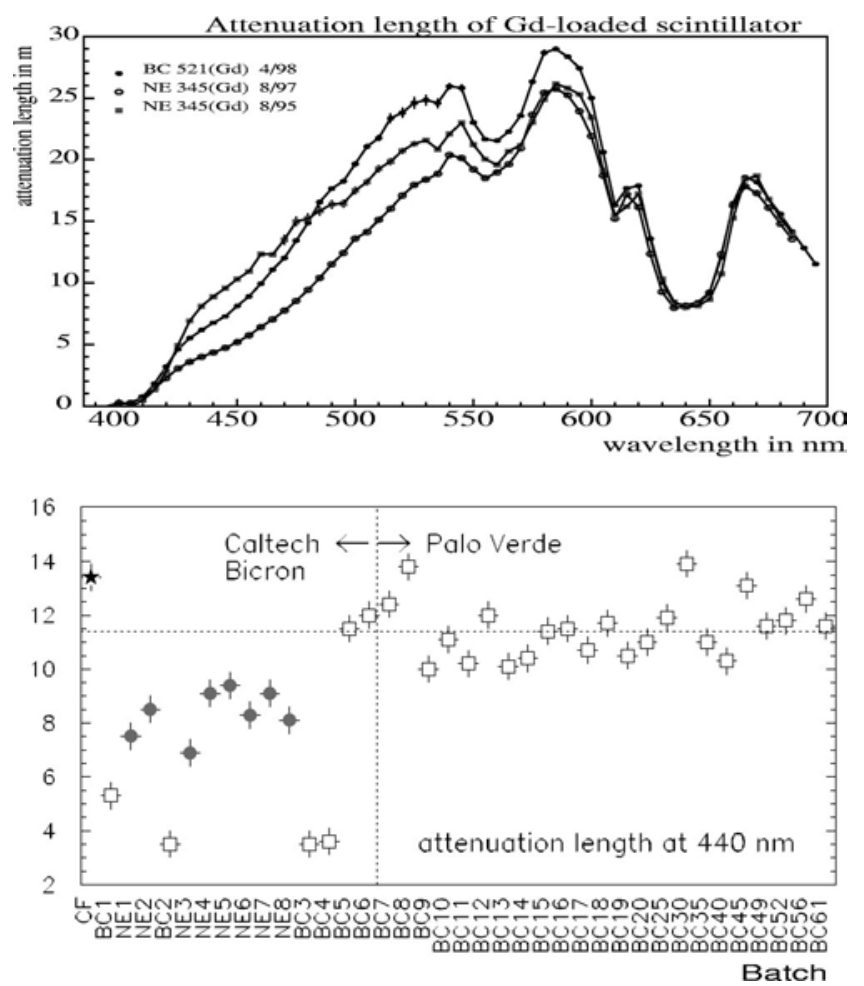

FIG. 13. Initial light attenuation length in the PALO VERDE scintillator: Top, attenuation length as a function of wavelength in three preproduction batches; bottom, attenuation length at $\lambda=440 \mathrm{~nm}$ for the initial batches of scintillator. Production batches are to the right of the vertical dashed line, while on the left we show various test batches not used in the detector. The first point (CF) refers to a standard (non-Gdloaded) scintillator fluid. Typical production scintillator had an attenuation length better than $11 \mathrm{~m}$.

the Gd compound in solution), with 2,5-diphenyloxazole (PPO) as the primary fluor. This scintillator had an H:C ratio of $\simeq 2$ and a light yield of $56 \%$ of anthracene, with a typical light attenuation length greater than $11 \mathrm{~m}$ at $\lambda=440 \mathrm{~nm}$ (Piepke et al., 1999).

The time stability of the same scintillator is shown in Fig. 14, in which the light attenuation curve for one cell and the effective attenuation length for all cells are presented as measured three times, at one-year intervals. The $12 \%$ loss in the first year decreases to $3 \%$ in the second, possibly indicating a gradual stabilization.

The time variation of the attenuation length $\lambda_{\mathrm{Gd}}$ of the Gd-loaded scintillator at CHOOZ, regularly measured in the detector, is well fitted by the empirical function

$$
\lambda_{\mathrm{Gd}}(t)=\frac{\lambda_{0}}{1+\alpha t},
$$

which accounts for the observed exponential decrease of the signal with time. Results of the fit are shown in Fig. 15.

The properties of the Gd-loaded and unloaded veto liquid scintillators developed for $\mathrm{CHOOZ}$ are presented in Table I.
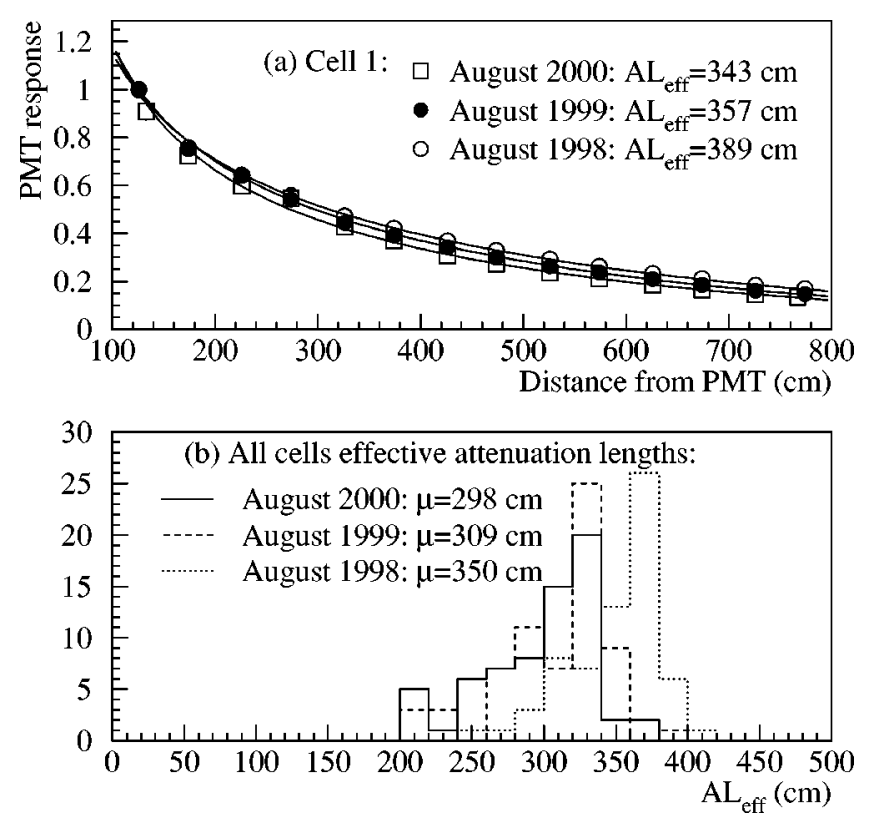

FIG. 14. Evolution of scintillation light attenuation length in the PALO VERDE scintillator during the two years of detector life: (a) the attenuation curve for scintillation events at different locations along one of the 9-m-long cells; (b) effective attenuation values for all detector cells. Note that the shorter value of the effective attenuation length reflects the nontrivial optics of the cells.

\section{A. $\mathrm{CHOOZ}$}

The CHOOZ detector was built at distances of 1115 and $998 \mathrm{~m}$ from the two reactors of the new CHOOZ power plant of Électricité de France in the Ardennes region of France. The plant, shown in Fig. 16, has a total

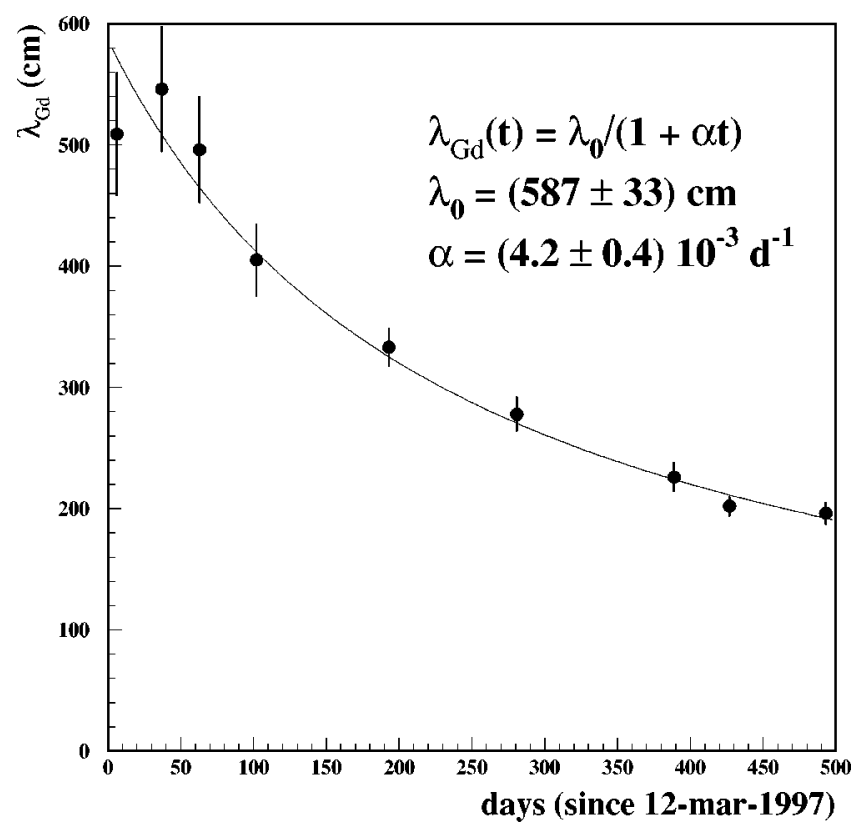

FIG. 15. Light attenuation length $\lambda_{\mathrm{Gd}}$ of the CHOOZ Gdloaded scintillator vs time with the best-fit function superimposed. 
TABLE I. Main properties of the liquid scintillators used in the CHOOZ experiment.

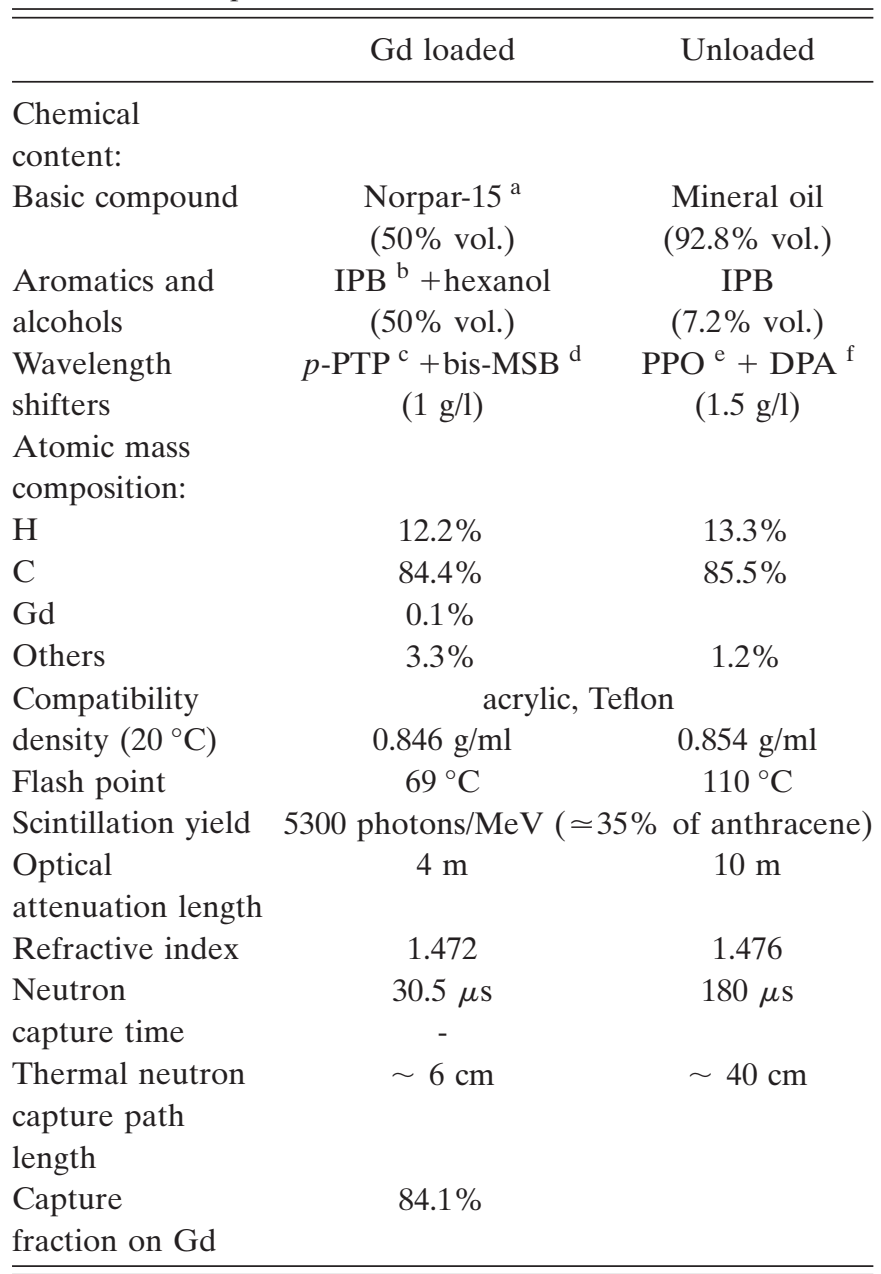

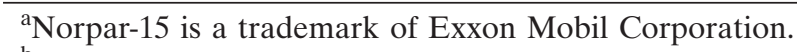

${ }^{\mathrm{b}}$ Monoisopropylbiphenyl.

${ }^{\mathrm{c}} p$-Terphenyl.

${ }^{\mathrm{d}} p$-Bis $(0$-methylstyryl)benzene.

2,5-Diphenyloxazole.

f 9,10 -Diphenylanthracene.

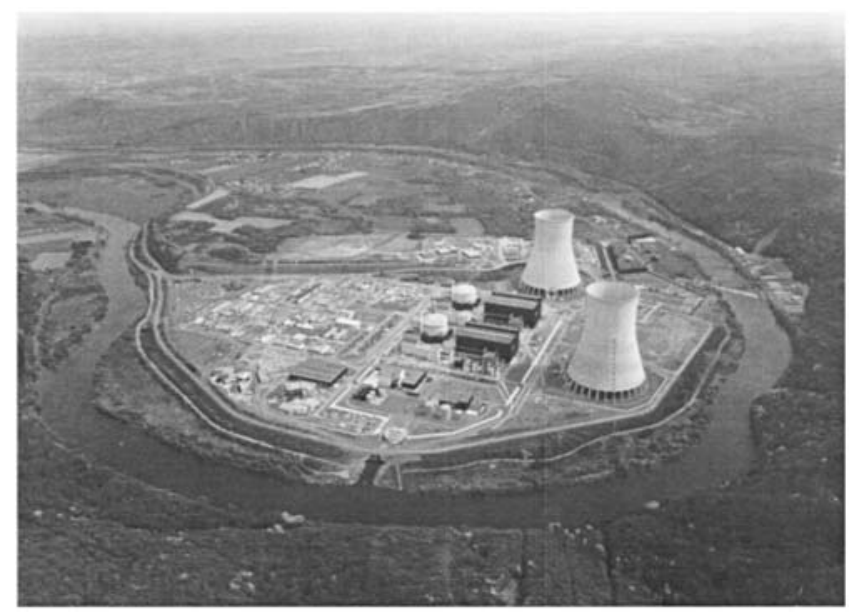

FIG. 16. Aerial view of the $\mathrm{CHOOZ}$ power plant. The detector is located in a tunnel under the hills on the bottom right of the photograph.
TABLE II. Summary of the CHOOZ data-taking conditions.

\begin{tabular}{lcc}
\hline \hline & Time (h) & $\int W_{t h} d t\left(\mathrm{GWh}_{t h}\right)$ \\
\hline Total run & 8761.7 & \\
Live & 8209.3 & \\
Dead & 552.4 & 8295 \\
Reactor 1 ON only & 2058.0 & 4136 \\
Reactor 2 ON only & 1187.8 & 8841 \\
Both reactors ON & 1543.1 & 0 \\
Both reactors OFF & 3420.4 & \\
\hline \hline
\end{tabular}

thermal power of $8.5 \mathrm{GW}_{t h}$, and the two reactors reached full power in May and August, 1997, respectively. The experiment took data from April 1997 until July 1998, under the conditions specified in Table II.

The apparatus, schematically shown in Fig. 17, consisted of a central volume of scintillator with a mass of 5 tons, where $\bar{\nu}_{e}$ 's were detected. This scintillator was contained in an acrylic vessel (region 1) that separated it from a 70-cm-thick shielding layer of mineral oil (region 2). 192 eight-inch photomultiplier tubes (PMT's) were mounted onto a steel vessel that, in turn, mechanically and optically isolated the central detector from the outer veto counter. The central detector had a photocathode coverage of $15 \%$ and a light yield of $\sim 130$ photoelectrons/MeV (Baldini et al., 1996). The 90-ton veto scintillator was at least $80 \mathrm{~cm}$ thick and was read out with two rings of 24 eight-inch PMT's; the outer containment tank was painted with white reflective paint. An outer layer (75 cm thick) of low-activity sand provided primary shielding from the rock.

Laser flashers were installed to monitor the detector performance, and radioactive sources could be inserted into the central region of the detector through special pipes. The detector energy response was calibrated daily with ${ }^{60} \mathrm{Co},{ }^{252} \mathrm{Cf}$, and $\mathrm{AmBe} \gamma$ and $n$ sources in order to

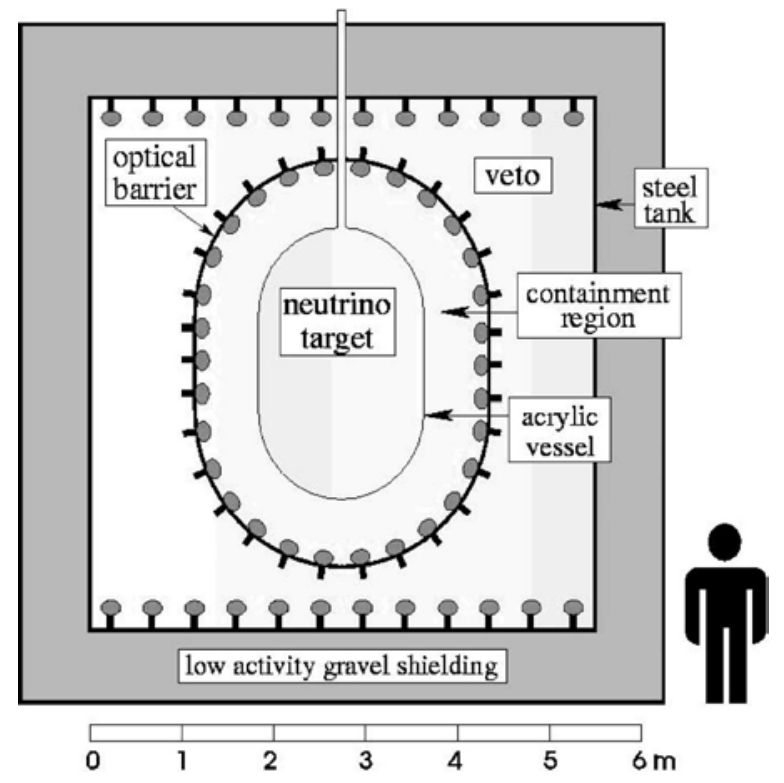

FIG. 17. Schematic drawing of the $\mathrm{CHOOZ}$ detector. 
${ }^{252} \mathrm{Cf}$ source, $\mathrm{z}=0$
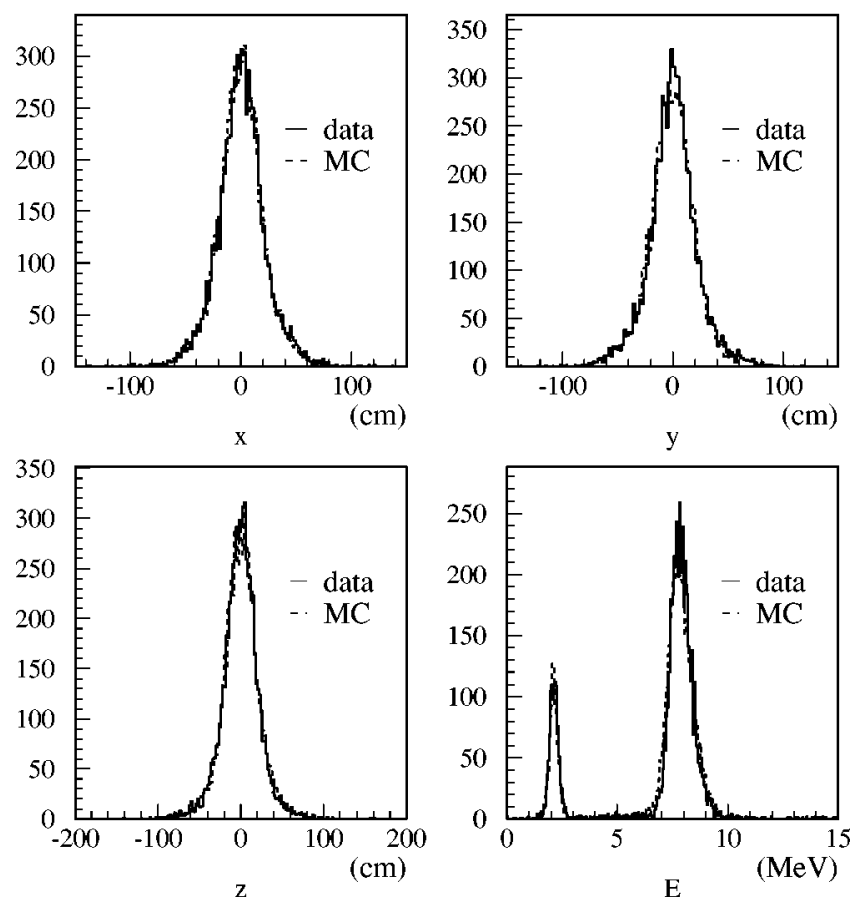

FIG. 18. Visible energy and position reconstructed for a calibration ${ }^{252} \mathrm{Cf}$ source placed inside the $\mathrm{CHOOZ}$ detector: top left, $x$ position; top right, $y$ position; bottom left, $z$ position; bottom right, total energy. $\mathrm{MC}=$ Monte Carlo simulation.

track the aging of the scintillator, the detector efficiency, and the energy calibration.

As an example we show in Fig. 18 the results of a $\mathrm{Cf}$ calibration run with the source placed in the middle of the detector. The data are compared with a Monte Carlo simulation for the reconstruction of the $x, y$, and $z$ positions and total energy in the detector. Both the peaks for $n$ captures on $p(2.2 \mathrm{MeV})$ and $\mathrm{Gd}(8 \mathrm{MeV})$ are clearly visible. The very good energy resolution $[\sigma(E) / E=5.6 \%$ at $8 \mathrm{MeV}]$ allows one to verify that the $8-\mathrm{MeV}$ peak is in fact the superposition of a $7.77-\mathrm{MeV}$ line with $77 \%$ weighting from capture on ${ }^{157} \mathrm{Gd}$ (energy shifted from $7.94 \mathrm{MeV}$ because of scintillator saturation effects) and an 8.31-MeV line with $23 \%$ weighting from capture on ${ }^{155} \mathrm{Gd}$ (energy shifted from $8.54 \mathrm{MeV}$ ). The fit to these two Gaussians gives $\chi^{2} / \mathrm{DOF}=67.6 / 55$, while the fit to a single Gaussian is very poor with $\chi^{2} / \mathrm{DOF}$ $=875 / 58$. The position resolution was found to be $\sigma_{x}$ $=\sigma_{y}=\sigma_{z}=17.5 \mathrm{~cm}$.

\section{B. PALO VERDE}

The PALO VERDE experiment was built at the Palo Verde Nuclear Generating Station, the largest nuclear plant in the Americas, $\sim 80 \mathrm{~km}$ west of Phoenix, in the Arizona desert. The total thermal power from three identical pressurized-water reactors was $11.6 \mathrm{GW}_{t h}$. Two of the reactors were located $890 \mathrm{~m}$ from the detector, while the third was at $750 \mathrm{~m}$. The shallow underground bunker housing the detector is shown at the time

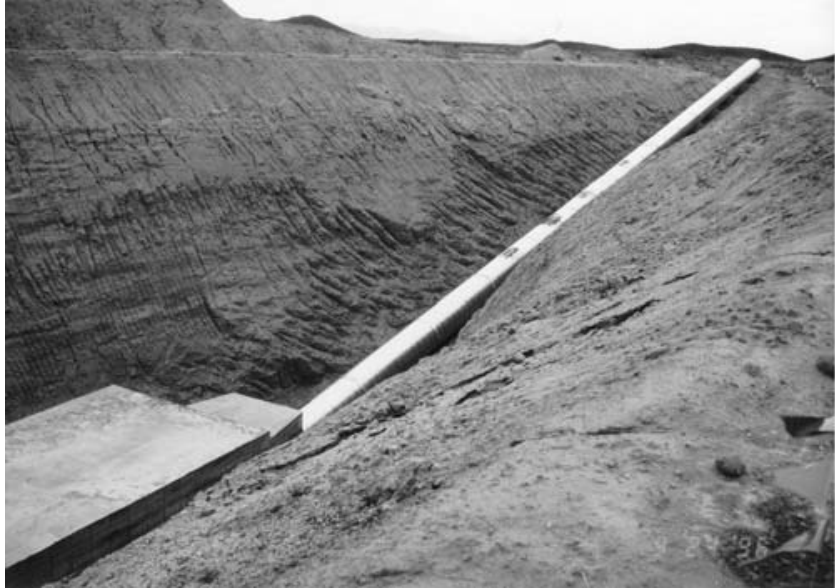

FIG. 19. The PALO VERDE underground laboratory at the time of construction (fall 1996).

of construction in Fig. 19. In total 350.5 days of $\bar{\nu}_{e}$ data were collected at PALO VERDE in the period between October 1998 and July 2000, covering four scheduled refueling outages as indicated in Fig. 11. Of these, 242.2 days were at full power, while the reactor at $750 \mathrm{~m}$ was off for 21.8 days and the reactors at $890 \mathrm{~m}$ were off for 86.5 days. Such data were complemented by frequent calibration runs.

The fiducial mass, segmented for active background rejection, consisted of 66 acrylic tanks filled with $0.1 \%$ Gd-loaded scintillator and arranged as shown in Fig. 20. Each cell was $9 \mathrm{~m}$ long, with a $12.7 \times 25.4-\mathrm{cm}^{2}$ cross section, and was viewed by two 5-inch photomultiplier tubes, one at each end. A $\bar{\nu}_{e}$ is identified by space- and time-correlated $e^{+}$and $n$ signals. Positrons deposited their energy in a scintillator cell and annihilated, yielding two 511-keV $\gamma$ 's that, in general, would be detected in different cells, giving a triple coincidence. Neutrons thermalized and were captured in Gd, giving a $\gamma$-ray shower of $8 \mathrm{MeV}$ total energy, also detected in more than one cell. The central detector was surrounded by a 1-m-thick water shield to moderate background neutrons produced by muons outside the detector and to absorb $\gamma$ 's from the laboratory walls. Outside of the water tanks were 32 large liquid scintillator counters and two endcaps to veto cosmic muons. The rate of cosmic muons was approximately $2 \mathrm{kHz}$. The pattern of muons traveling through veto counters and their timing relative to the central detector hits were recorded for subsequent off-line analysis. The central detector was equipped with a system of tubes that allowed the insertion of calibration sources in the small spaces between cells. In addition, a set of blue light-emitting diodes (LED's) and optical fibers was used to produce flashes of light inside each of the cells. In order to reduce natural radioactivity, all building materials for the detector were carefully selected, including the aggregate (marble) used in the concrete of the underground laboratory.

Both the positron and the neutron were triggered by a triple coincidence requiring at least one cell above a 


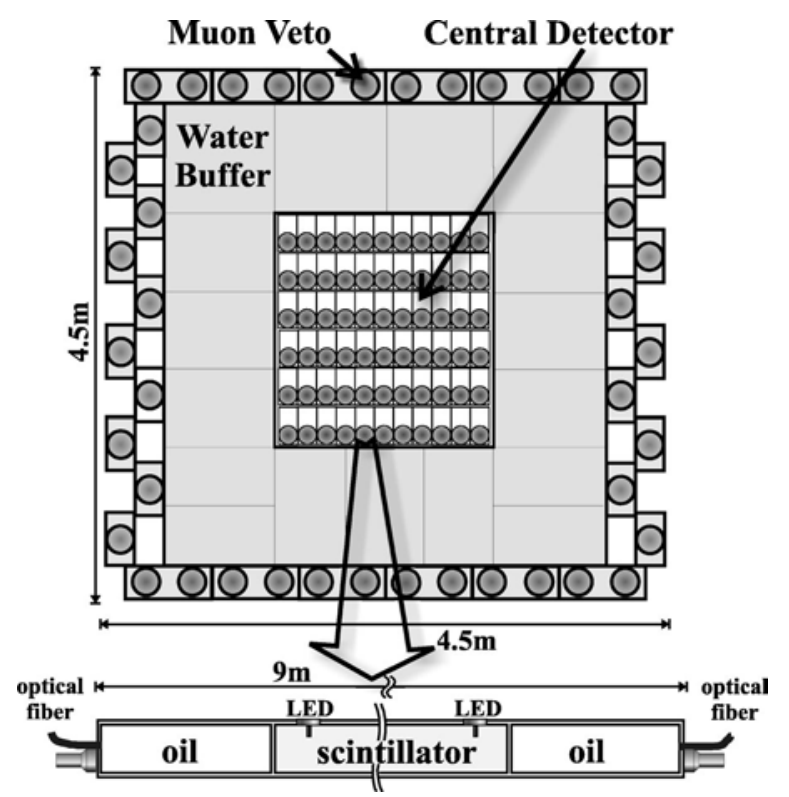

FIG. 20. Schematic view of the PALO VERDE neutrino detector.

"high" threshold set at $\sim 600 \mathrm{keV}$ (positron ionization or neutron capture cascade) and two cells above a "low" threshold set at $\sim 40 \mathrm{keV}$ (Compton scattering from annihilation photons or neutron capture cascade tails). The triple coincidences were required to be within $3 \times 5$ matrices of cells anywhere in the detector as recognized by a custom-made trigger processor (Gratta et al., 1997).

The efficiency calibration was based upon a primary measurement performed a few times per year with a calibrated ${ }^{22} \mathrm{Na} e^{+}$source and an AmBe neutron source. The ${ }^{22} \mathrm{Na}$ source mimicked the effects of the positron from the $\bar{\nu}_{e}$ interaction by providing annihilation radiation and a $1.275-\mathrm{MeV}$ photon that simulated the $e^{+}$ionization in the scintillator. The source was placed at 18 positions in the detector deemed to be representative of different conditions. The results of this procedure were then rescaled to the $e^{+}$case using a Monte Carlo simulation. The neutron detection efficiency was measured by scanning the detector with the AmBe source after tagging the 4.4-MeV $\gamma$ associated with the neutron emission with a miniaturized $\mathrm{NaI}(\mathrm{Tl})$ counter. Other calibrations, used to measure the detector energy response, were performed using the Compton edges from ${ }^{137} \mathrm{Cs}$, ${ }^{65} \mathrm{Zn}$, and ${ }^{228} \mathrm{Th}$ sources. The same Th source was also used more frequently to track the scintillator transparency, as already shown in Fig. 14. Weekly runs of the fiber-optic and LED flasher systems were used to monitor the gain and linearity of photomultipliers and the timing/position relationship along the cells, respectively.

Since the energy deposition of the 511-keV $\gamma$ 's in one cell has a sharply falling spectrum (Compton scattering), it was vital to have the lowest possible "low" thresholds in the trigger and to understand the behavior of such thresholds with great accuracy. This second task was complicated by the fact that the trigger used voltage amplitudes, while only charge from integrating analog-todigital converters (ADC's) was available offline. For this
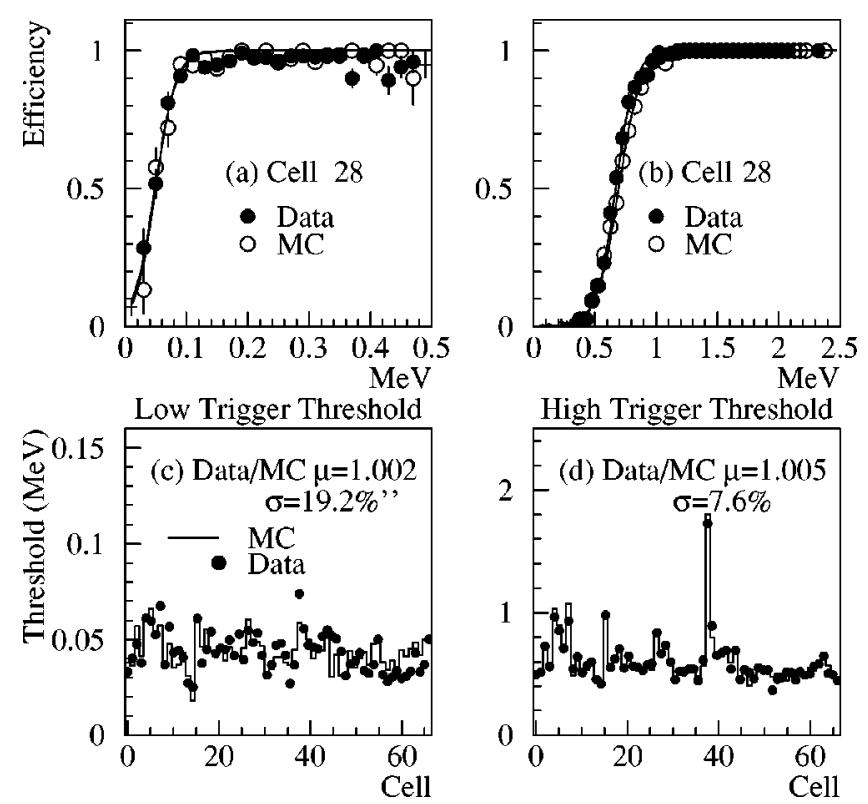

FIG. 21. A comparison of the trigger thresholds at PALO VERDE from data and a Monte Carlo simulation. The data were taken with a ${ }^{22} \mathrm{Na}$ source at the center of each cell. The panels (a) and (b) show the efficiency of the trigger thresholds (left, low; right, high) for a typical cell as a function of energy deposited; the bottom panels (c) and (d) show the energy at $50 \%$ efficiency for (left) low and (right) high thresholds in all 66 cells.

reason the detector simulation included a detailed description of the signal development in time. This code correctly described the shape of pulses, taking into account scintillator light yield, attenuation length, and deexcitation time; photomultiplier rise and fall time and gain; and event position along a cell. The simulation of the detector response to the ${ }^{22} \mathrm{Na}$ source is shown in Fig. 21 and correctly describes the $40-\mathrm{keV}$ threshold position to within $1.4 \mathrm{keV}$ and the $600-\mathrm{keV}$ threshold position to within $2.6 \mathrm{keV}$, resulting in uncertainties on the positron and neutron efficiencies of $4 \%$ and $3 \%$, respectively.

\section{Backgrounds}

There are generally two types of background affecting long-baseline reactor experiments in which the signal is based on the correlated $e^{+}, n$ signature: uncorrelated hits from cosmic rays and natural radioactivity and correlated ones from cosmic- $\mu$-induced neutrons. The first type can be measured by studying the time difference between positronlike and neutronlike parts of an event. More insidious are cosmic- $\mu$-induced neutrons that present the same time and space correlation between prompt and delayed parts of the event as in $\bar{\nu}_{e}$. Such events are schematically shown in the PALO VERDE detector in Fig. 22. Neutrons are produced by cosmic- $\mu$ spallation and capture on the materials outside the veto counter. Both production mechanisms can result in either neutron thermalization and capture, in which the thermalization process fakes the prompt triple coincidence, or secondary neutron production, in which one of 


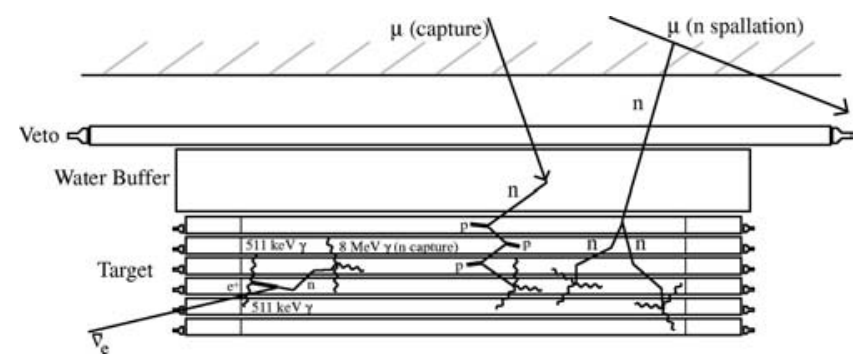

FIG. 22. Schematic view of two types of cosmic- $\mu$-induced backgrounds and a signal event (far left) in the PALO VERDE detector. Neutrons are produced by cosmic- $\mu$ spallation (right) and capture (left) on the materials outside the veto counter. Both can result in either neutron thermalization and capture (left), in which the thermalization process fakes the prompt triple coincidence, or secondary neutron production (right), in which one of the captures fakes the prompt triple coincidence.

the captures fakes the prompt triple coincidence. Conceptually the same situation holds for $\mathrm{CHOOZ}$ (and KAMLAND, as will be discussed later), although differences in overburden and the simpler scheme of coincidence numerically change the relative importance of different backgrounds. It is useful to point out that direct neutrons from the reactors have a totally negligible effect at the distances discussed here.

Both experiments preselect $\bar{\nu}_{e}$ candidates by requiring an appropriate topology (in space and time) for the prompt and delayed parts of each event and their relative position. Such cuts ensure that the spatial and temporal extents of the events are compatible with the $\bar{\nu}_{e}$ hypothesis and that events are well contained and mea- sured in the detector. A general classification in terms of signal and different backgrounds can be conveniently made by studying the correlation between prompt and delayed energy in $\mathrm{CHOOZ}$ for such preselected samples, as shown in Fig. 23. The region marked "B" in the figure contains cosmic-ray muons stopping in the detector after entering undetected by the veto counter. Both prompt energy (muon ionization) and delayed energy (Michel electron) are large. Indeed, events in region $\mathrm{B}$ show a fast time correlation between prompt and delayed part, consistent with the muon lifetime. Region $\mathrm{C}$ is populated by the muon-spallation events discussed above: large prompt energy deposit from proton recoils during neutron thermalization is accompanied by a fixed 8-MeV energy deposit characteristic of neutron capture. Regions $\mathrm{A}$ and $\mathrm{D}$ are populated by random coincidences of natural radioactivity hits, sometimes including a highenergy proton recoil from neutron scattering in the delayed part (region A). Neutrino candidates populate the region framed by the darker line, as can be seen by comparison between the scatter plots with reactors on and off.

The time elapsed between the prompt and delayed parts of the events is shown in Fig. 24 for the PALO VERDE data. We note that the process of $n$ capture in the segmented detector requires the sum of two exponentials to fit properly. This is due to the fact that a fraction of the neutrons stop, after thermalization, in passive materials (mainly acrylic for PALO VERDE) where there is no Gd and the capture is a slower process. While the Monte Carlo simulation gives a good fit with two exponentials, for the data a third exponential with a longer time constant is needed in the fit. This
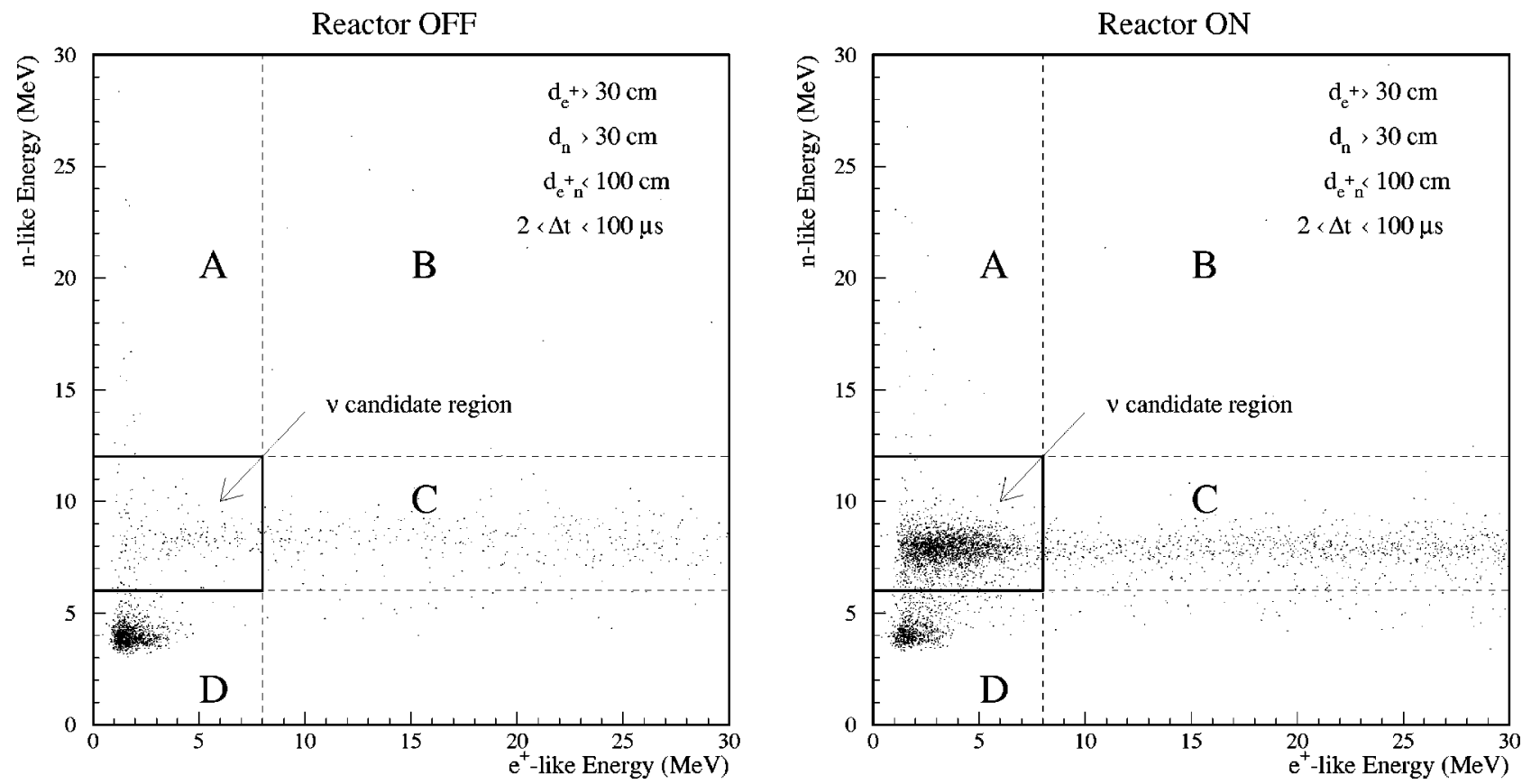

FIG. 23. Delayed energy ( $n$-like energy) vs prompt ( $e^{+}$-like energy) energy in preselected CHOOZ events. The selection cuts are listed in the figure. Left, reactor OFF; right, reactor ON. A description of the event types in the different regions of the plot is given in the text. 

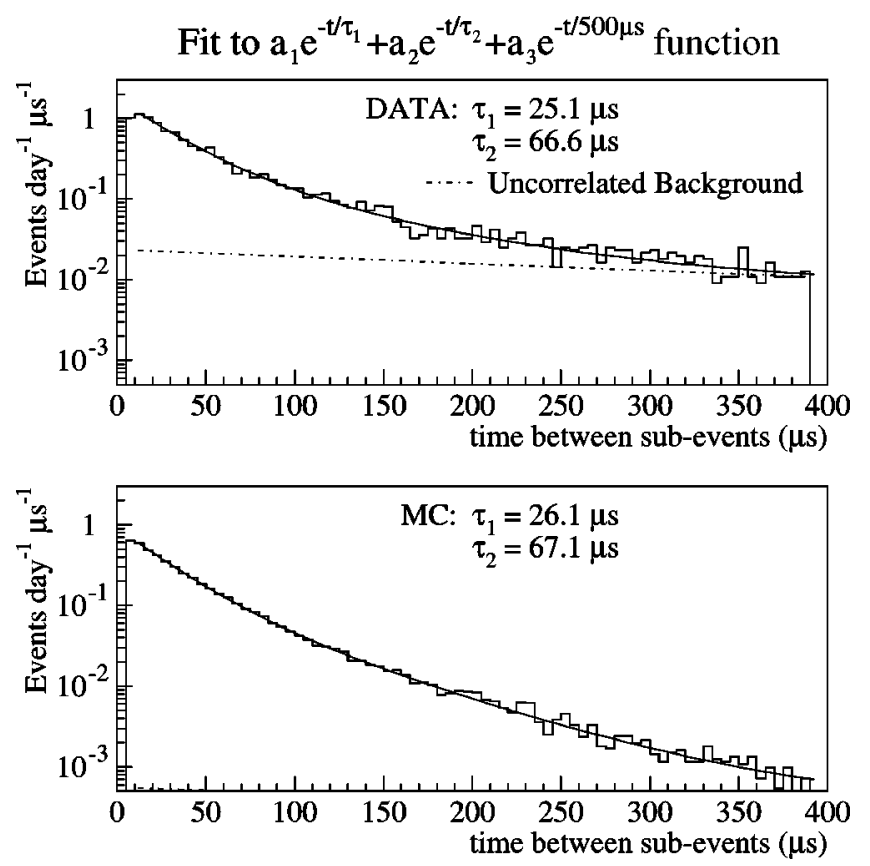

FIG. 24. Time elapsed between the prompt and delayed parts of events in PALO VERDE data (above) and a Monte Carlo simulation (below). The simulated data are fit to two exponentials. Real data are fit to three exponentials, the third of which accounts for the random background. For an explanation of the $\tau_{1}$ and $\tau_{2}$ values, see text.

third exponential accounts for events initiated by uncorrelated background, which arises when the delayed part is triggered by cosmic rays crossing the detector with a $2-\mathrm{kHz}$ rate. Timing cuts are applied by both experiments to ensure that events are consistent with a neutron capture. In addition events are rejected for a period of time following tracks detected in the veto counters. This last cut is particularly important at PALO VERDE, where the cosmic-ray rate is high.

The availability for $\mathrm{CHOOZ}$ of data at zero power and with the reactors ramping up provides an independent way to check the magnitude of signal and back-

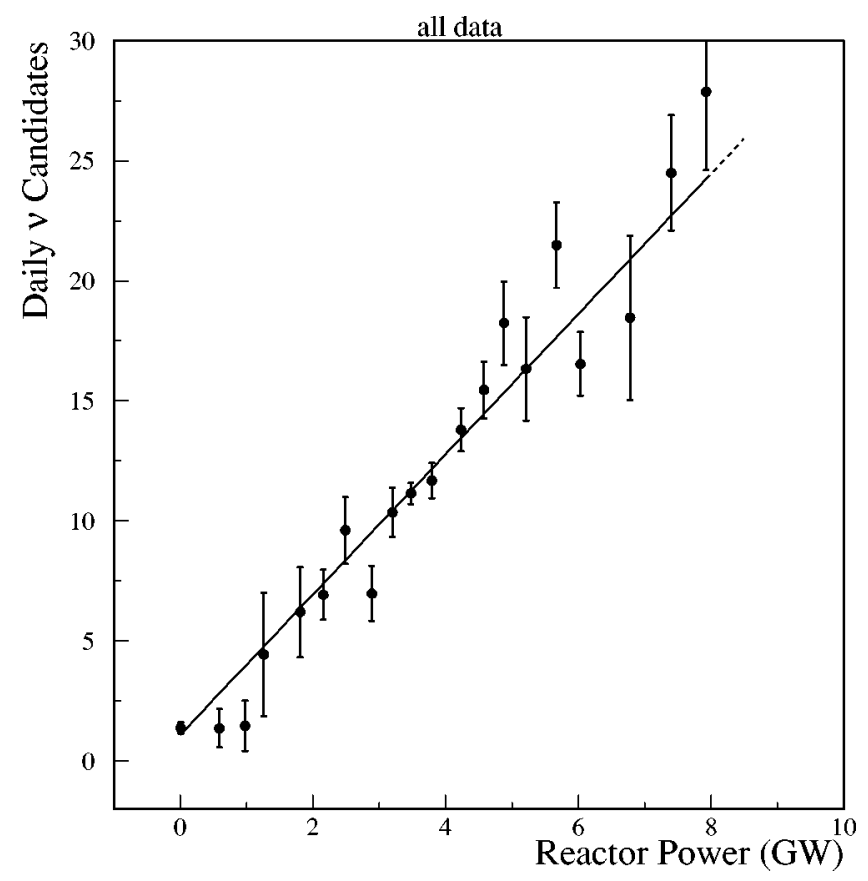

FIG. 25. $\mathrm{CHOOZ} \bar{\nu}_{e}$ rate during reactor commissioning. The background at reactor $\mathrm{OFF}$ is $1.1 \pm 0.25$ events/day.

ground. The fitting procedure proceeds as follows. For each run the predicted number of neutrino candidates $\bar{N}_{i}$ results from the sum of a signal term, linearly depending on the reactor's effective power $W^{*}$ and the background, assumed to be constant and independent of power; ${ }^{5}$ therefore,

$$
\bar{N}_{i}=\left(B+W_{i}^{*} X\right) \Delta t_{i}
$$

where the index $i$ labels the run number, $\Delta t_{i}$ is the corresponding live time, $B$ is the background rate, and $X$ is the positron yield per unit power averaged over the two reactors.

The results are listed in Table III for three data-taking periods corresponding to threshold readjustments. The data are also shown in compact form in Fig. 25.

A simple subtraction of the $e^{+}$spectra with reactor on

TABLE III. Summary of the likelihood fit parameters for the three data-taking periods at CHOOZ.

\begin{tabular}{lccc}
\hline \hline Period & 1 & 2 & 3 \\
\hline Starting date & 7 April 1997 & 30 July 1997 & 12 January 1998 \\
Runs & $579 \rightarrow 1074$ & $1082 \rightarrow 1775$ & $1778 \rightarrow 2567$ \\
Live time (h) & 1831.3 & 2938.8 & 3268.4 \\
Reactor-off time (h) & 38.9 & 539.5 & 2737.2 \\
$\int^{\quad} W d t(\mathrm{GWh})$ & 7798 & 10636 & 2838 \\
$B$ (counts $/ d)$ & & & \\
$X$ (counts/d GW) & $1.25 \pm 0.6$ & $1.22 \pm 0.21$ & $2.2 \pm 0.14$ \\
$\chi^{2} / \mathrm{DOF}$ & $2.60 \pm 0.17$ & $2.60 \pm 0.09$ & $2.51 \pm 0.17$ \\
$N_{\nu}$ (counts $\left./ d\right)$ & $136 / 117$ & $135 / 154$ & $168 / 184$ \\
$($ at full power) & $24.8 \pm 1.6$ & $24.8 \pm 0.9$ & $24.0 \pm 1.6$ \\
\hline \hline
\end{tabular}

\footnotetext{
${ }^{5}$ The "effective" power $W^{*}$ is a fictitious thermal power corresponding to both reactors located at the reactor 1 site, and thus providing $9.55 \mathrm{GW}$ at full operating conditions and at the start of reactor operation.
} 

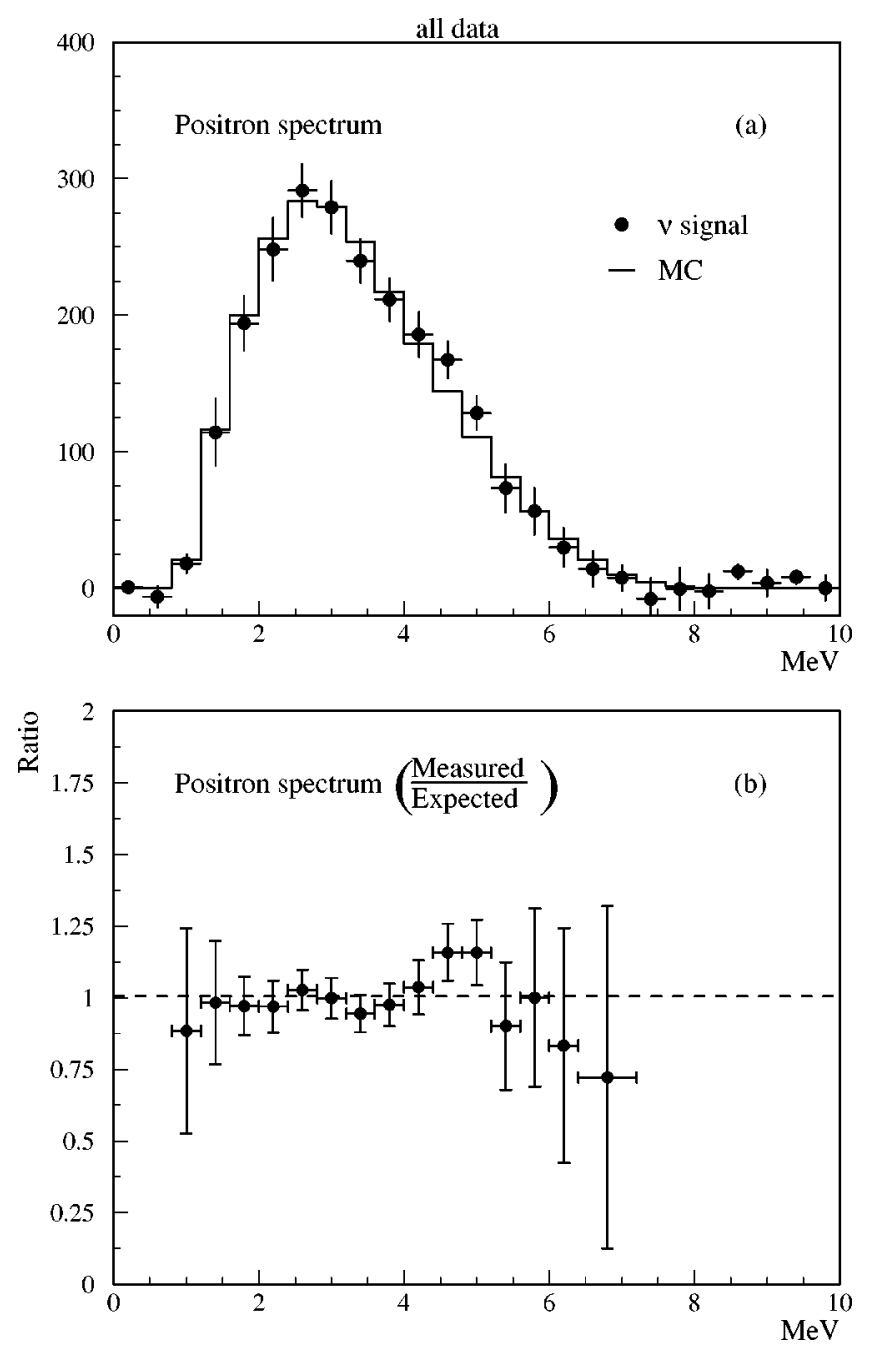

FIG. 26. Background-subtracted positron energy spectrum in the $\mathrm{CHOOZ}$ data. Error bars represent statistical errors only. The solid histogram in (a) represents the expectation for the case of no oscillations. The ratio between the two curves is shown on the bottom panel in (b).

and off gives for $\mathrm{CHOOZ}$ the spectrum shown in Fig. 26. The comparison of the observed distribution with the expected one for the no-oscillation case already shows very good agreement.

The same procedure can be repeated for PALO VERDE using the thermal power excursions due to refueling. However, in this case this technique substantially magnifies the errors since (1) the periods of low power still have about $2 / 3$ of the full flux, so that in the subtraction most of the signal is lost, and (2) the statistical errors are dominated by the relatively short periods of low power. In addition, for any experiment, the background subtraction method will give a correct result only if special attention is paid to the data quality, guaranteeing in particular that the efficiencies for signal and background are accurately known and remain constant through the experiment.

An alternative method (Wang et al., 2000) was developed for the PALO VERDE analysis starting from the evidence that, for their depth and detector configura- tion, the dominant correlated background has at least two neutrons, each triggering the detector with its capture. Such intrinsic symmetry can be used to cancel most of the background directly from data and compute the remaining components from Monte Carlo simulations. This technique makes the best possible use of the statistical power of all data collected. The rate of candidate events after all cuts can be written as $N=B_{\text {unc }}+B_{\text {nn }}$ $+B_{\mathrm{pn}}+S_{\nu}$, where the contribution of the uncorrelated $B_{\text {unc }}$, two-neutron $B_{\mathrm{nn}}$, and other correlated backgrounds $B_{\mathrm{pn}}$ are explicitly represented, along with the $\bar{\nu}_{\mathrm{e}}$ signal $S_{\nu}$. The dominant background $B_{\mathrm{nn}}$ (along with $\left.B_{\text {unc }}\right)$ is symmetric under exchange of subevents, so that an event selection with the requirements for the prompt and delayed event parts swapped will result in a rate $N^{\prime}=B_{\text {unc }}+B_{\mathrm{nn}}+\epsilon_{1} B_{\mathrm{pn}}+\epsilon_{2} S_{\nu}$. Here $\epsilon_{1}$ and $\epsilon_{2}$ account for the different efficiency for selecting asymmetric events after the swap. One can then calculate $N-N^{\prime}$ $=\left(1-\epsilon_{1}\right) B_{\mathrm{pn}}+\left(1-\epsilon_{2}\right) S_{\nu}$, where the efficiency correction $\epsilon_{2} \simeq 0.2$ can be estimated from the $\bar{\nu}_{e}$ Monte Carlo simulation.

The PALO VERDE group found that the processes of $\mu$ spallation in the laboratory walls and capture of the $\mu$ 's that are not tagged by the veto counter contributed to $\left(1-\epsilon_{1}\right) B_{\mathrm{pn}}$, while other backgrounds were negligible. Using Monte Carlo simulation, they obtained (1 $\left.-\epsilon_{1}\right) B_{\mathrm{pn}}=-0.9 \pm 0.5 d^{-1}$ for $\mu$ spallation in the 1998 data set and $-1.3 \pm 0.6 d^{-1}$ in the 1999 data set; the same figures for $\mu$ capture are $0.6 \pm 0.3 d^{-1}$ in 1998 and $0.9 \pm 0.5 d^{-1}$ in 1999 . This represents only a small correction to $N-N^{\prime}$, since the error on $B_{\mathrm{pn}}$ is reduced by the fact that $\epsilon_{1}$ is close to 1 . While the Monte Carlo model is accurate for the capture process, in the case of spallation the broad range of spectral indexes for the $n$ recoil energy reported in the literature was simulated (Wang et al., 2000). The average of different predictions is then used for $B_{\mathrm{pn}}$, while the spread is used as an extra systematic error. Since no $\bar{\nu}_{\mathrm{e}}$ signal is present above 10 $\mathrm{MeV}$, the observed integrated rate above such energy is used as a normalization of the Monte Carlo simulation. The rate of neutrons produced by muon spallation has been measured at PALO VERDE (Boehm et al., 2000c), and the dependence of the neutron spallation yield on depth has been analyzed by Wang et al. (2001).

The PALO VERDE results obtained in this way are shown in Table IV for different running periods. Clearly in this case there is also good agreement with the nooscillation hypothesis.

\section{Event reconstruction techniques}

The identification of the neutrino signal and the rejection of the background in reactor neutrino experiments depends on the accuracy of the event energy and position determinations and on the spatial and time correlations of the detected positron and neutron. Event reconstruction in a segmented detector like PALO VERDE is relatively simple. However, event characterization in single-vessel detectors like CHOOZ or KAMLAND, which are viewed by photomultipliers placed at the ves- 
TABLE IV. Summary of results from the PALO VERDE experiment (Boehm et al., 2001). Uncertainties are statistical only. $N$, $N^{\prime}$, and $\left(1-\epsilon_{1}\right) B_{\mathrm{pn}}$ are measured rates, while background and $R_{\nu}\left(\bar{\nu}_{e}\right.$ interaction rate) are efficiency corrected (assuming that the background events are measured with the same efficiency as the signal). $R_{\text {calc }}$ is the calculated $\bar{\nu}_{e}$ interaction rate for the nooscillation hypothesis. See text for other notations.

\begin{tabular}{|c|c|c|c|c|c|c|c|c|}
\hline \multirow{2}{*}{$\begin{array}{l}\text { Period } \\
\text { Reactor }\end{array}$} & \multicolumn{2}{|c|}{1998} & \multicolumn{2}{|c|}{ 1999-I } & \multicolumn{2}{|c|}{ 1999-II } & \multicolumn{2}{|c|}{2000} \\
\hline & On & $890 \mathrm{~m}$ off & On & $750 \mathrm{~m}$ off & On & $890 \mathrm{~m}$ off & On & $890 \mathrm{~m}$ off \\
\hline Duration (d) & 30.4 & 29.4 & 68.2 & 21.8 & 60.4 & 29.6 & 83.2 & 27.5 \\
\hline Efficiency (\%) & 8.0 & 8.0 & 11.5 & 11.6 & 11.6 & 11.6 & 10.9 & 10.8 \\
\hline$N\left(d^{-1}\right)$ & $39.6 \pm 1.1$ & $34.8 \pm 1.1$ & $54.9 \pm 0.9$ & $45.1 \pm 1.4$ & $54.2 \pm 0.9$ & $49.4 \pm 1.3$ & $52.9 \pm 0.8$ & $43.1 \pm 1.3$ \\
\hline$N^{\prime}\left(d^{-1}\right)$ & $25.1 \pm 0.9$ & $21.8 \pm 0.9$ & $33.4 \pm 0.7$ & $32.0 \pm 1.2$ & $32.5 \pm 0.7$ & $32.6 \pm 1.0$ & $30.2 \pm 0.6$ & $30.4 \pm 1.1$ \\
\hline$\left(1-\epsilon_{1}\right) B_{\mathrm{pn}}\left(d^{-1}\right)$ & 0.88 & 0.89 & 1.11 & 1.11 & 1.11 & 1.11 & 1.07 & 1.07 \\
\hline Background $\left(d^{-1}\right)$ & $292 \pm 11$ & $255 \pm 10$ & $265 \pm 6$ & $266 \pm 10$ & $256 \pm 6$ & $265 \pm 9$ & $249 \pm 5$ & $272 \pm 9$ \\
\hline$R_{\nu}\left(d^{-1}\right)$ & $202 \pm 19$ & $182 \pm 18$ & $212 \pm 10$ & $124 \pm 17$ & $214 \pm 11$ & $161 \pm 15$ & $237 \pm 10$ & $129 \pm 16$ \\
\hline$R_{\text {calc }}\left(d^{-1}\right)$ & 216 & 154 & 218 & 129 & 220 & 155 & 218 & 154 \\
\hline
\end{tabular}

sel surface, requires refined reconstruction methods making the best use of the PMT charge and time information. The relative importance of the time and charge information in optimizing the detector spatial resolution (which also affects the energy resolution) depends on several factors: the size of the vessel, the distance between PMT's, their time resolution, the fiducial target volume, and the scintillator light yield, relaxation time, and attenuation length. In CHOOZ, the relatively small detector volume and the small distance between the neutrino target and the PMT's made the charge information dominant in the precise event characterization. In the case of larger-volume detectors, filled with non-Gdloaded scintillator, like KAMLAND (Alivisatos et al., 1998) or the solar neutrino experiment BOREXINO (Alimonti et al., 1998, 2000), the time information gains importance.

As an example of a minimization algorithm for event reconstruction based on the PMT-measured charge, we describe the one used for CHOOZ. The standard algorithm uses a maximum-likelihood method to reconstruct the energy $E$ and the vertex $\vec{x}$ of an event. The likelihood is defined as the joint Poissonian probability of observing a measured distribution of photoelectrons over 24 "patches," each grouping 8 adjacent PMT's, for given $(E, \vec{x})$ coordinates in the detector. Therefore, for an event occurring at time $t$ after the start of data taking, one can build a likelihood function as follows:

$$
\mathcal{L}(N ; \bar{N})=\prod_{j=1}^{24} P\left[N_{j} ; \bar{N}_{j}(E, \vec{x}, t)\right]=\prod_{j=1}^{24} \frac{\bar{N}_{j}^{N_{j}}}{N_{j} !} e^{-\bar{N}_{j}},
$$

where $N_{j}$ is the observed number of photoelectrons and $\bar{N}_{j}$ the expected number for the $j$ th patch given an event $(E, \vec{x}, t)$. The reason for using Poissonian instead of Gaussian statistics is due to the frequent occurrence of low-energy events with a low number of photoelectrons detected by some PMT patches. The predicted number of photoelectrons for each patch is computed by considering a local deposit of energy, resulting in a number of visible photons that are tracked to each PMT through the differently attenuating region 1 (Gd-doped) and region 2 scintillators. Therefore

$$
\bar{N}_{j}=\alpha E \eta \sum_{k=1}^{8} \frac{\Omega_{j k}(\vec{x})}{4 \pi} \exp \left(-\frac{d_{1}^{j k}(\vec{x})}{\lambda_{\mathrm{Gd}}(t)}-\frac{d_{2}^{j k}(\vec{x})}{\lambda_{\mathrm{Hi}}}\right)
$$

where $E$ is the ionization energy deposited in the scintillators, $\alpha$ is the light yield of the scintillator, $\eta$ is the average PMT quantum efficiency, $\Omega_{j k}$ is the solid angle subtended by the $k$ th PMT from the event position, $d_{1}^{j k}$ is the light path length in region $1, d_{2}^{j k}$ is the light path length in region $2, \lambda_{\mathrm{Gd}}$ is the attenuation length in the region 1 scintillator, and $\lambda_{\mathrm{Hi}}$ is the attenuation length in the region 2 scintillator.

The solid angle is approximated by the expression

$$
\Omega_{j k}=2 \pi\left(1-\frac{d_{j k}}{\sqrt{d_{j k}^{2}+r_{P M T}^{2} \cos \theta}}\right),
$$

where $r_{P M T}$ is the PMT photocathode radius, $d_{j k}=d_{1}^{j k}$ $+d_{2}^{j k}$, and $\theta$ is the angle between the event-PMT direction and the inward unit vector normal to the PMT surface.

Instead of using Eq. (35), as is usually the case for problems involving the maximum-likelihood method, it is more convenient to use the theorem of the "likelihood ratio test" for goodness of fit to convert the likelihood function into the form of a general $\chi^{2}$ statistic (Eadie et al., 1971). If one assumes $N_{j}$ to be the best estimate of the true (unknown) photoelectron distribution and can form the likelihood ratio $\lambda$ defined by

$$
\lambda=\frac{\mathcal{L}(N ; \bar{N})}{\mathcal{L}(N ; N)},
$$

the "likelihood ratio test" theorem states that the "Poissonian" $\chi^{2}$, defined by

$$
\chi^{2}=-2 \ln \lambda=2 \sum_{j=1}^{24}\left[\bar{N}_{j}-N_{j}+N_{j} \ln \left(\frac{N_{j}}{\bar{N}_{j}}\right)\right],
$$

asymptotically obeys a chi-square distribution (Baker and Cousins, 1984). It is easy to prove that the minimization of $\chi^{2}$ is equivalent to maximization of the likelihood function, so that the $\chi^{2}$ statistic may be useful both for estimating the event characteristics and for goodness-of-fit testing. 
TABLE V. Origin and magnitude of systematic errors in PALO VERDE and CHOOZ. Note that the two experiments offer different breakdowns of their systematics. For simplicity we do not show the systematics for the PALO VERDE ONOFF analysis.

\begin{tabular}{|c|c|c|}
\hline Systematic & $\begin{array}{c}\mathrm{CHOOZ} \\
(\%)\end{array}$ & $\begin{array}{c}\text { PALO VERDE } \\
(\%)\end{array}$ \\
\hline$\sigma\left(\bar{\nu}_{e}+p \rightarrow n+e^{+}\right)$ & 1.9 & \\
\hline Number of $p$ 's in target & 0.8 & \\
\hline$W_{t h}$ & 0.7 & \\
\hline Energy absorbed per fission & 0.6 & \\
\hline Total rate prediction & 2.3 & 2.1 \\
\hline$e^{+}$trigger efficiency & & 2.0 \\
\hline$n$ trigger efficiency & & 2.1 \\
\hline $\bar{\nu}_{e}$ selection cuts & & 2.1 \\
\hline$\left(1-\epsilon_{1}\right) B_{\mathrm{pn}}$ estimate & & 3.3 \\
\hline Total $\bar{\nu}_{e}$ efficiency & 1.5 & 4.9 \\
\hline Total & 2.7 & 5.3 \\
\hline
\end{tabular}

The CERN MINUIT package (James, 1994) was used to minimize Eq. (39). The starting value for the $i$ th coordinate was based on the charge asymmetries measured by initially grouping the PMT's into only six "superpatches," referred to the detector frame axes; it was defined according to

$$
x_{i 0}=\frac{\sqrt{Q_{+}^{i}}-\sqrt{Q_{-}^{i}}}{\sqrt{Q_{+}^{i}}+\sqrt{Q_{-}^{i}}} D^{i}, \quad i=1,2,3,
$$

where the indices + and - refer to the opposite superpatches of the $i$ th axis, and $D^{i}$ is the half size of the detector along that axis. Once the $x_{i 0}$ corresponding to the starting position is known, the starting energy value is obtained from Eq. (36) after replacing $\vec{x}$ with $\vec{x}_{0}$ and $\bar{N}_{j}$ with $N_{j}$. Examples of the results obtained by this procedure are shown in Fig. 18.

\section{E. Results and systematics}

A summary of systematic errors for both $\mathrm{CHOOZ}$ and PALO VERDE is given in Table V. The systematic error given for $\mathrm{CHOOZ}$ should probably be considered as some sort of ultimate limit for reactor-based oscillation experiments, at least when only one detector is present. Indeed the intrinsically high efficiency $(\simeq 70 \%)$ of the homogeneous detector, together with the unique opportunity of studying the zero-power case, are important advantages (in comparison the efficiency of the larger but segmented PALO VERDE detector is $\simeq 11 \%$ ).

The (energy-averaged) ratio between $\bar{\nu}_{e}$ detected and expected was found to be

$$
R=1.01 \pm 0.028(\text { stat }) \pm 0.027 \text { (syst) } \quad \mathrm{CHOOZ}
$$

and

$$
\begin{aligned}
R= & 1.01 \pm 0.024(\text { stat }) \\
& \pm 0.053 \text { (syst) } \quad \text { PALO VERDE }
\end{aligned}
$$

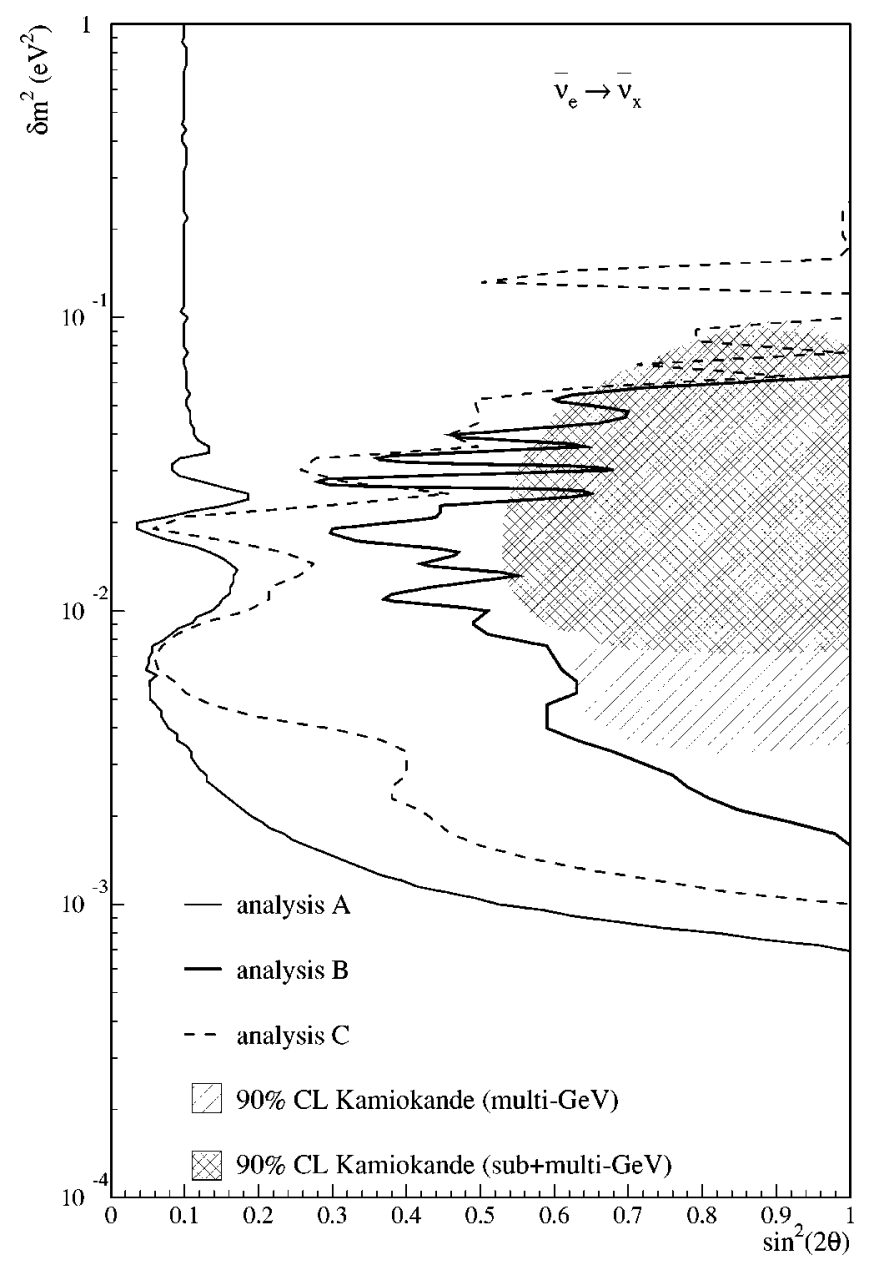

FIG. 27. Limits on mass difference and mixing angle from CHOOZ (90\% C.L.) obtained with the unified approach (Feldman and Cousins, 1998). Analysis A (thin solid line) refers to the curve obtained by a fit to the backgroundsubtracted spectrum in which both shape and normalization are used. Analysis C (dashed line) uses only the shape of the spectrum. Finally, analysis B (heavy solid line) uses the difference of baselines between the two reactors $(\Delta L=116.7 \mathrm{~m})$. While in this last case most systematics cancel, statistical errors are larger and the $\Delta m^{2}$ sensitivity is rather poor due to the short baseline difference. The Kamiokande $\nu_{e}-\nu_{\mu}$ atmospheric neutrino result, which motivated the present work, is also shown.

in both cases consistent with 1 .

Both experiments were able to exclude $\bar{\nu}_{e}-\bar{\nu}_{\mathrm{x}}$ oscillations as dominant cause of the atmospheric neutrino anomaly. This is evident from the exclusion contours obtained using the unified approach (Feldman and Cousins, 1998) and shown in Fig. 27 for CHOOZ and Fig. 28 for PALO VERDE.

\section{F. Are smaller mixing angles within experimental reach?}

The current data on neutrino oscillations suggest the need to include at least three neutrino flavors when studying results from experiments. As discussed in the Introduction, the most general approach would involve 


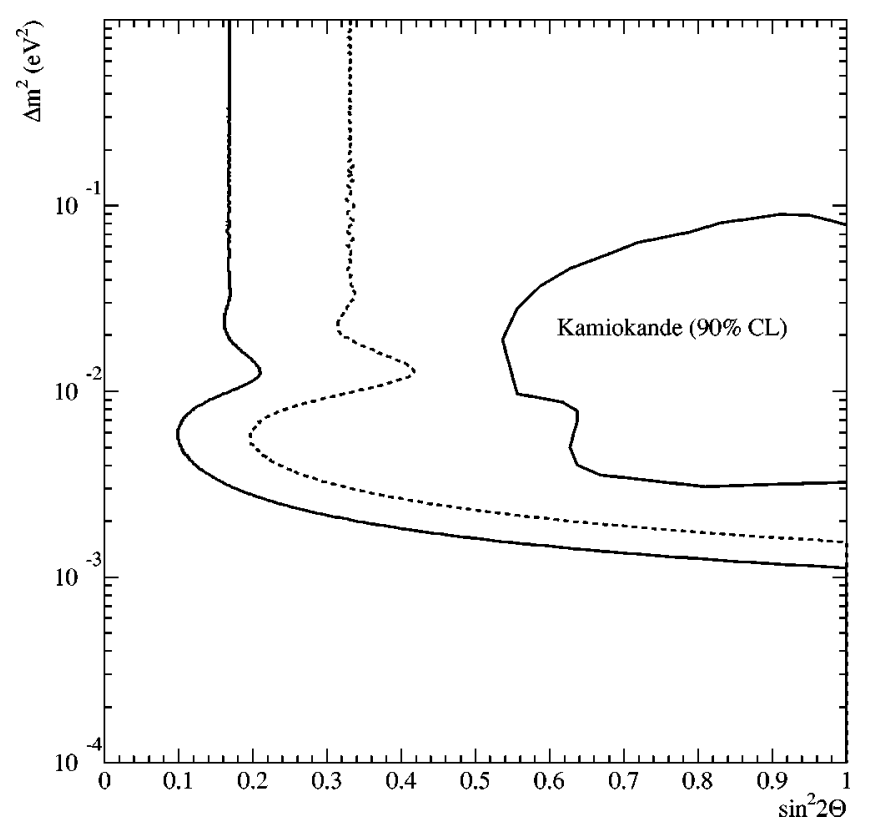

FIG. 28. Limits on mass difference and mixing angle from PALO VERDE at $90 \%$ C.L. (Boehm et al., 2001). The solid curve is obtained with the swap background subtraction method described in the text, while the dashed curve is obtained using the reactor power changes to estimate and subtract the background. The Kamiokande $\nu_{\mu}-\nu_{e}$ atmospheric neutrino result, which motivated the present work, is also shown for illustration.

five unknown parameters: three mixing angles and two independent mass differences. However, an intermediate approach consists of a simple generalization of the two-flavor scenario, assuming that $m_{3}^{2} \gg m_{1}^{2}, m_{2}^{2}$ (i.e., $\Delta m_{13}^{2}=\Delta m_{23}^{2}=\Delta m^{2}$, while $\left.\Delta m_{12}^{2} \simeq 0\right)$. This scenario is obviously compatible with the evidence based on the atmospheric neutrino anomaly $\left(\Delta m^{2} \sim 3 \times 10^{-3} \mathrm{eV}^{2}\right)$ and the solar neutrino deficit $\left(\Delta m^{2}<10^{-4} \mathrm{eV}^{2}\right)$. In such a case the mixing angle $\theta_{12}$ becomes irrelevant, and one is left with only three unknown quantities: $\Delta m^{2}, \theta_{13}$, and $\theta_{23}$. With this parametrization and assuming $C P T$ symmetry $\left(\nu_{e} \rightarrow \nu_{x}\right.$ probability is the same as $\left.\bar{\nu}_{e} \rightarrow \bar{\nu}_{x}\right)$, the $\bar{\nu}_{e}$ disappearance is governed by

$$
P\left(\bar{\nu}_{e} \rightarrow \bar{\nu}_{x}\right)=\sin ^{2} 2 \theta_{13} \sin ^{2} \frac{\Delta m^{2} L}{4 E_{\nu}},
$$

while $\nu_{\mu} \rightarrow \nu_{\tau}$ oscillations, responsible for the atmospheric neutrino results in this scenario, are described by

$$
P\left(\nu_{\mu} \rightarrow \nu_{\tau}\right)=\cos ^{4} \theta_{13} \sin ^{2} 2 \theta_{23} \sin ^{2} \frac{\Delta m^{2} L}{4 E_{\nu}} .
$$

An analysis of the atmospheric neutrino data based on these assumptions has been performed (Okamura, 1999) and its results are shown in Fig. 29 for the $\nu_{e}$ disappearance channel. One can see that, while the relevant region of the mass difference $\Delta m^{2}$ is determined by the atmospheric neutrino data, the mixing angle $\theta_{13}$ is not constrained very much. Here the reactor-based neutrino oscillation experiments play a decisive role.

The determination of the angle $\theta_{13}$ has obvious impor-

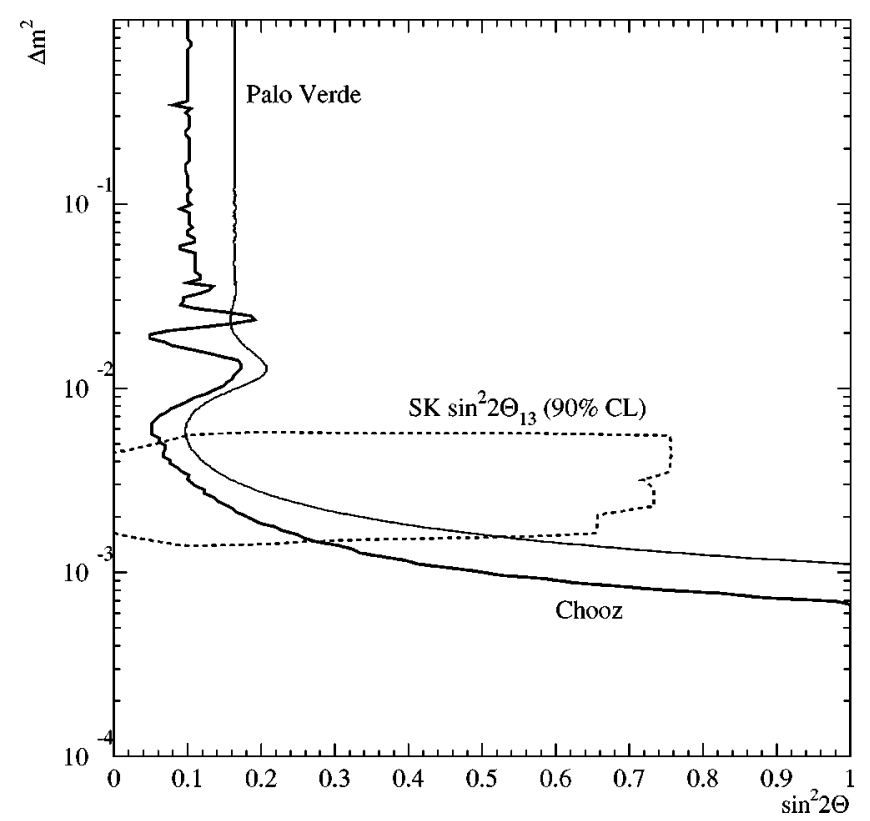

FIG. 29. Exclusion plot showing the allowed region of $\theta_{13}$ and $\Delta m^{2}$ based on the Super-Kamiokande preliminary analysis (the region inside the dotted curve). The regions excluded by the neutrino reactor experiments are to the right of the corresponding continuous curves.

tance not only for the structure of the lepton mixing matrix $U$ but for the observability of $C P$ violation in the lepton sector, as stressed in the Introduction. If $\theta_{13}$ vanishes or is very small, no $C P$ violation effects are observable in the lepton sector. Moreover, for vanishing $\theta_{13}$ and with three neutrinos only, the lepton mixing is radically simplified. The electron neutrino is then simply

$$
\nu_{e}=\cos \theta_{12} \nu_{1}+\sin \theta_{12} \nu_{2}
$$

while the $\nu_{\mu}$ and $\nu_{\tau}$ neutrinos become superpositions of $\nu_{3}$ and the corresponding orthogonal combination of $\nu_{1}$ and $\nu_{2}$. It is therefore interesting to ask whether reactor-based experiments can be extended to address regions of even smaller mixing parameter $\sin ^{2} 2 \theta_{13}$.

A simple inspection of Table $\mathrm{V}$ shows that, using the CHOOZ systematics, if all flux and cross-section-related errors could be set to zero, one would be left with an error of $\simeq 1.5 \%$. Hence, assuming a detector large enough to produce negligible statistical error, the total error would shrink from the present $3.9 \%$ to $1.5 \%$.

This scenario is considered by Mikaelyan (2000), who proposes using an underground reactor at Krasnoyarsk in Russia as a source and two identical detectors placed at distances of $\simeq 1100 \mathrm{~m}$ and $\simeq 250 \mathrm{~m}$. The interesting feature of the Krasnoyarsk site is that there are substantial facilities available underground, with an overburden of $\sim 600$ mwe, twice the depth of CHOOZ. Indeed it might even be conceivable to locate the detectors on rail-cars and periodically switch their position to further reduce some of the systematics related to detector efficiency. The proposal discusses the use of 50 tons of Gdloaded scintillator for each of the two identical homogeneous detectors, so that the far detector would collect 50 events/day (the thermal power of the reactor is in this 
case lower than at CHOOZ or PALO VERDE). The background is estimated by Mikaelyan (2000) to be about 5 events/day or less.

This proposal estimates that such an experiment could reach a sensitivity in mixing strength of better than 0.02 in the $\Delta m^{2}$ region relevant for atmospheric neutrinos. While the idea certainly looks interesting, it would be useful to explore how practical it is in general to push the errors of the absolute $\bar{\nu}_{e}$ flux to the $1 \%$ domain, even with the measurements considered here. Note also that the Krasnoyarsk reactors, according to the Gore-Chernomyrdin ${ }^{6}$ agreement, are supposed to be shut down for recoring in the not very distant future for some period of time.

\section{EXPLORING THE SOLAR $\nu$ ANOMALY ON EARTH: KAMLAND}

While historically solar neutrinos provided the first hint of oscillations, there is a consensus today that the strongest evidence for oscillation is the atmospheric neutrino anomaly. Indeed, the zenith-angle dependence of the anomaly has substantially helped to eliminate explanations not based on some property of neutrinos themselves, and the advent of KEK-to-Kamioka (K2K), to be followed soon by MINOS and the CERN-to-Gran Sasso programs (Wojcicki, 2001a, 2001b), is bringing the study of oscillations in this regime to a laboratory setting with both source and detector well under control.

In the case of solar neutrinos, none of the effects that would generally be considered "smoking guns" for oscillations has as yet clearly emerged from the data [although the juxtaposition of SNO (Ahmad, 2001) and Super-Kamiokande (S. Fukuda, 2001a) comes close]. The exploration "in a laboratory setting" of the problem is made particularly challenging by the huge $L / E_{\nu}$ required. It is probably a safe prediction that it will take a very long time before an accelerator-based experiment will be able to tackle the solar neutrino problem. However, the very low energy of reactor neutrinos makes a reactor-based oscillation experiment able to reach the large-mixing-angle MSW solution possible-albeit rather challenging. While the analysis of current and future solar neutrino experiments presumably will help to decide which of the possible solutions is the right one, we find the chance to study solar neutrino oscillations in the laboratory extremely compelling. Note that in contrast to atmospheric neutrinos, for which it turned out that electron neutrinos are not involved in the dominant mixing, the solar neutrino problem, if due to oscillations, obviously involves $\nu_{e}$ disappearance. Therefore, unless $\bar{\nu}_{e}$ 's behave drastically differently from $\nu_{\mathrm{e}}$ 's (which would be a worthwhile discovery anyway, signaling the breakdown of $C P T$ symmetry), a reactor experiment is

\footnotetext{
6"US-Russian Plutonium Production Agreement is Signed," statement by the White House Office of the Vice President, 23 September 1997.
}

an exact replica of the astrophysical experiment, only built on earth.

\section{A. Nuclear reactors in Japan}

The "easier" solution of the solar neutrino problem (large-mixing-angle MSW) is shown in Fig. 1. In order to completely explore this solution, one needs a $\Delta m^{2}$ sensitivity of at least $10^{-5} \mathrm{eV}^{2}$ at a large mixing angle. As is now customary, we refer to Fig. 3 as a first step in designing our experiment: we see that $\mathrm{a} \approx 100$-km baseline is needed, which drives the power $\times$ fiducial-mass product into the $10^{8}-\mathrm{MW}_{t h} \times$ tons range. Clearly a large detector has to be used in conjunction with very many nuclear reactors. A cursory look at the placement of nuclear power plants on the earth (Fig. 30) reveals that such an experiment could only be placed in Europe, the eastern United States, or Japan.

There are 16 commercial nuclear power plants in Japan; their location is shown in Fig. 31. They supply about $1 / 3$ (or $130 \mathrm{GW}_{t h}$ ) of the total electric power in the country. At the Kamioka site there is an antineutrino flux of $\simeq 4 \times 10^{6} \mathrm{~cm}^{-2} \mathrm{~s}^{-1}$ (or $\simeq 1.3 \times 10^{6} \mathrm{~cm}^{-2} \mathrm{~s}^{-1}$ for $E_{\bar{v}}>1.8 \mathrm{MeV}$ ) from these reactors. Eighty percent of this flux derives from reactors at a distance between 140 and $210 \mathrm{~km}$, so that there is a limited range of baselines. The breakdown of this data by power plant (several plants have on site more than one reactor) is given in Table VI. We note that some $2 \%$ of the flux derives from power plants in South Korea (the Primorskaya plant in Russia is only in the planning stages) that will have to be included (albeit only as a crude estimate) to provide an exact flux prediction.

While Table VI assumes the nominal power for each of the cores, an average over one year, taking into account scheduled and unscheduled down times, gives an expected nonoscillation rate of $\simeq 750 \mathrm{kton}^{-1} \mathrm{yr}^{-1}$ for a $\mathrm{C}_{n} \mathrm{H}_{2 n+2}$ target. Although the signal is provided by a very large number of cores, it turns out that a modulation of the $\bar{\nu}_{e}$ flux is expected at KAMLAND (Alivisatos et al., 1998), thanks to the refueling and maintenance schedule of nuclear power plants in Japan. Such shutdowns, in fact, are concentrated in the fall and spring, when the power demand is lowest, as illustrated in Fig. 32. Hence, from the point of view of the tools available to study backgrounds, KAMLAND is in a situation very similar to that of PALO VERDE, with two dips in the flux from full to $\approx 2 / 3$ expected every year.

It is interesting to note that other artificial sources of low-energy $\bar{\nu}_{e}$ 's do not significantly contribute to the background in KAMLAND. The largest effect would be produced by a large nuclear-powered vessel while running its reactors at full power in the Toyama Bay, $50-100 \mathrm{~km}$ from the detector. In these circumstances the excess signal in KAMLAND would amount to $\sim 10 \%$ (Detwiler, 2000). Clearly, it is extremely unlikely that such conditions will occur for any significant period of time.

\section{B. Detector design}

The KAMLAND detector is housed in the cavity built for the Kamiokande detector under the summit of Mt. 


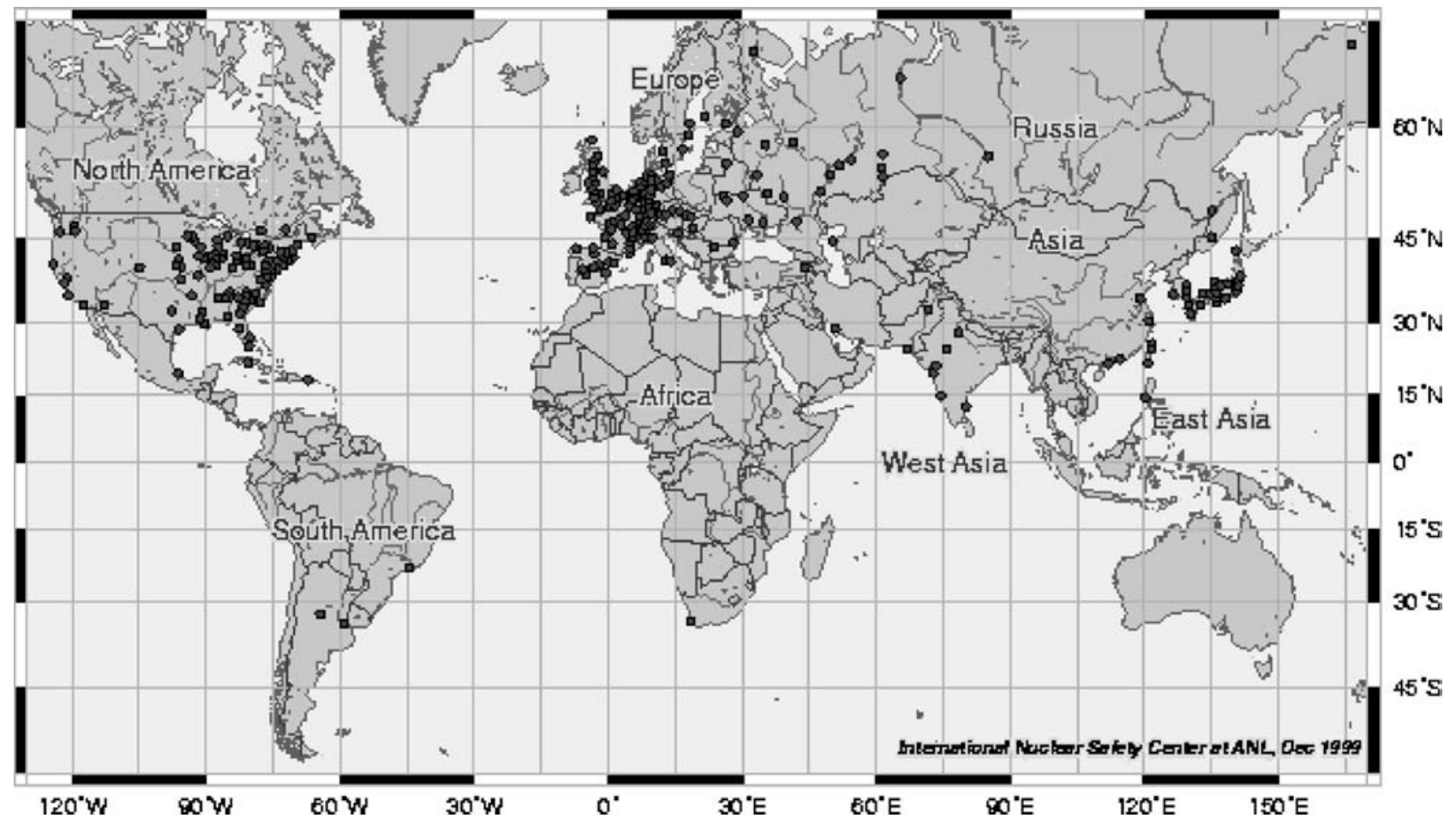

FIG. 30. Location of nuclear power plants in the world. Substantial concentrations of reactors are found in Europe, the eastern US, and Japan. (Note that the map, see http://www.insc.anl.gov/, contains a few plants that were either planned but never built or are no longer operational.)

Ikenoyama in the Japanese Alps, about $50 \mathrm{~km}$ south of the town of Toyama. The layout of the laboratory is shown in Fig. 33. The rock overburden is more than $1000 \mathrm{~m}$ in any direction, with an average rock density of $2.7 \mathrm{~g} / \mathrm{cm}^{3}$. The site is $500 \mathrm{~m}$ from Super-Kamiokande.

A cutout view of the KAMLAND detector is shown in Fig. 34. The fiducial volume consists of a sphere containing 1000 tons of liquid scintillator. The scintillator container is a thin plastic-walled balloon of $6.5-\mathrm{m}$ radius that is not supposed to take the weight of the scintillator but only to isolate it from an outer 2.5 -m-thick layer of nonscintillating, radiation-shielding fluid. The balloon is also designed to be impermeable to radon that mainly originates from $\mathrm{Th}$ and $\mathrm{U}$ contamination inside the PMT's glass. The buffer fluid and the liquid scintillator are contained and mechanically supported by a stainless steel spherical vessel that also provides the mechanical structure on which the photomultipliers for the fiducial volume are mounted. The sphere is solidly anchored inside the cylindrical rock cavity, and the space between them is filled with water and used as a veto Cerenkov counter. The scintillator, based on mineral oil and pseudocumene, is designed to achieve sufficient light yield and $n-\gamma$ discrimination by pulse-shape analysis, while complying with rather strict flammability requirements from the Kamioka mine. Given the cost and stability issues for a detector the size of KAMLAND, it was decided not to Gd-load the scintillator. As will be discussed later, simulations indicate that sufficient signal-to-noise ratio will be achieved with unloaded scintillator. Events will be localized inside the fiducial volume using the light intensity and propagation delays to the different photomultipliers so that large-area, fast tubes are required. While the veto counter will be read out using 20-in. photomultipliers dismounted from the Kamiokande detector, new, faster tubes with 17 -in. active photocathodes have been developed for KAMLAND in order to allow for proper vertex reconstruction from timing. Such tubes have an average transit-time spread of $\simeq 3 \mathrm{~ns}$ (to be compared to $\simeq 5 \mathrm{~ns}$ for the Kamiokande/Super-Kamiokande tubes). The central detector has a $30 \%$ photocathode coverage obtained using about 1280 seventeen-inch tubes complemented, for energy measurements, by 642 twenty-inch Kamiokande tubes. A spherical shell of acrylic panels (not shown in Fig. 34) is mounted at a radius immediately inside the position of the PMT's and is used as the primary barrier against radon migration into the active scintillator. A cylindrical stainless steel chimney of $3 \mathrm{~m}$ diameter protrudes from the top of the sphere to permit access to the central detector during installation. Buffer fluid and scintillator lines as well as calibration ports are mounted in the chimney along with all the electrical cabling.

The readout of KAMLAND is designed to provide wave-form analysis information for each of the PMT's in the detector with essentially no dead time for several consecutive events. This allows for clean event reconstruction and enables the off-line study of the prehistory of interesting events. For example, multiple neutron events, described above as the most dangerous background at PALO VERDE, will be fully reconstructed by KAMLAND. Similarly cosmogenic activation giving short-half-life nuclei will be clearly recorded. Deep digi- 


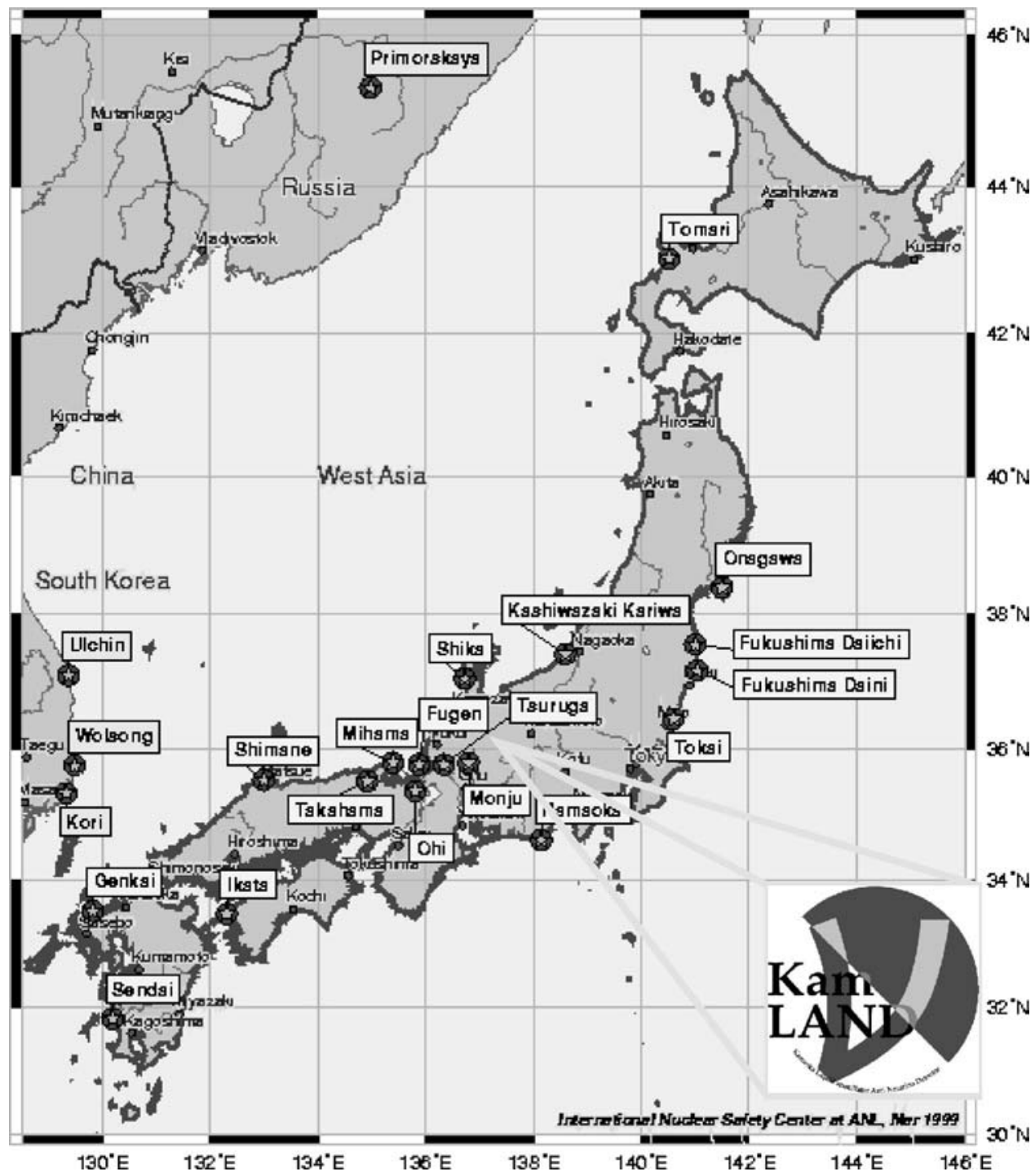

FIG. 31. Location of large nuclear power plants in Japan, Korea, and Far East Russia. (See the comment in the caption of Fig. 30.)

tal buffering will allow the detector to sustain a substantial burst of events like those expected from supernovae.

In Fig. 35 we show a phase of the central detector PMT installation that was concluded in September 2000. Scintillator filling started in spring 2001, and data taking at KAMLAND began January 22, 2002.

\section{Expected performance}

As in previous experiments, both random hits from natural radioactivity and correlated events from neutron production in cosmic-ray-muon spallation and capture contribute to the background for reactor $\bar{\nu}_{e}$ in KAMLAND. The results of a Monte Carlo full detector simulation using the measured Kamioka cosmic-ray flux and the activities of various components as sampled during construction are given in Table VII. For the purpose of this background estimate, $\mathrm{U}$ and $\mathrm{Th}$ contaminations in the scintillator of $10^{-14} \mathrm{~g} / \mathrm{g}$ have been assumed. Such a purity level has already been achieved in samples of the KAMLAND scintillator. Monte Carlo studies have shown that cosmogenic activation gives a negligible contribution to the background for doubles. A discussion of backgrounds for single signatures, not considered here, can be found in Alivisatos et al. (1998).

In Fig. 36 we show the predicted energy spectra for reactor neutrinos at KAMLAND for no oscillations and different oscillation parameters consistent with the large-mixing-angle MSW solar neutrino solution. We can use one of these curves and add to it fluctuations consistent with a 10/1 signal-to-noise ratio and three years of data to investigate the sensitivity of the experiment. Assuming that oscillations with $\Delta m^{2}=2$ $\times 10^{-5} \mathrm{eV}^{2}$ and $\sin ^{2} 2 \theta=0.75$ are indeed the cause of the solar neutrino anomaly, we obtain the measurement of the oscillation parameters shown in Fig. 37. On the other hand, no evidence for oscillation after three years of data would result in the exclusion curve shown in Fig. 1 and would rule out the large-mixing-angle MSW solution to the solar neutrino problem.

\section{Other physics with a very large low-energy $\bar{\nu}_{e}$ detector}

KAMLAND will be the largest detector specifically optimized to detect low-energy $\bar{\nu}_{e}$ 's with good efficiency 


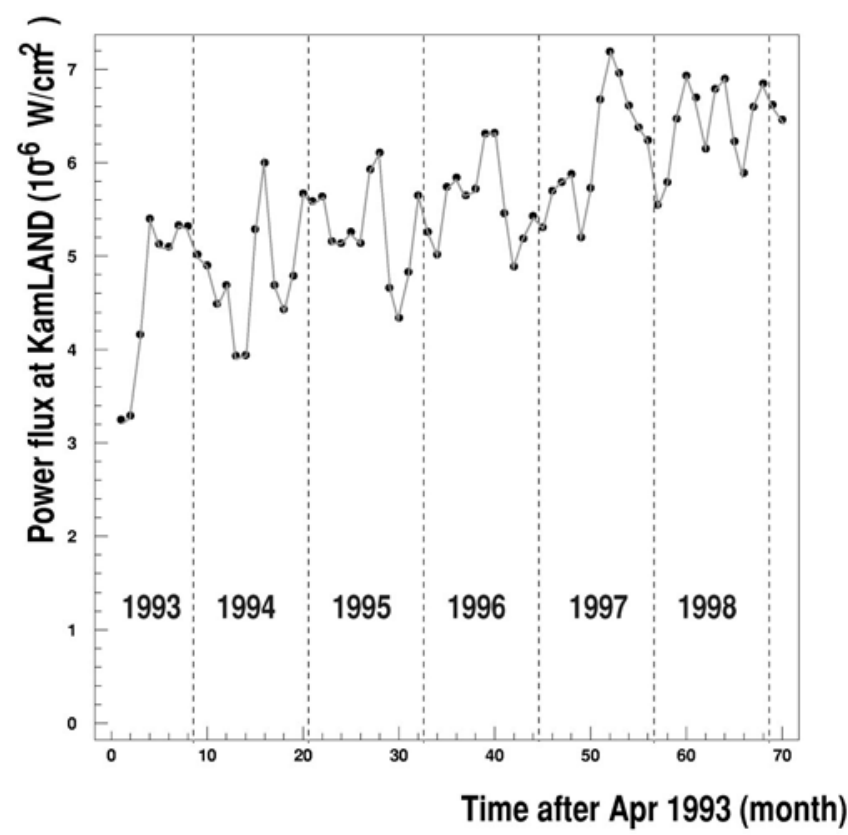

FIG. 32. Power-flux level at Kamioka from Japanese reactors as function of time. Low power periods in the fall and spring are alternated with peaks of high power in the summer and winter.

and low background. This opens the way for a number of interesting experiments beyond the measurement of oscillations from reactors. In addition, such a large detector with a low energy threshold can be used to directly measure neutrinos from the sun, assuming that backgrounds can be sufficiently reduced and understood
TABLE VI. List of relevant parameters for power reactors in Japan and South Korea.

\begin{tabular}{lccccc}
\hline \hline & \multicolumn{5}{c}{ No. } \\
Site & $\begin{array}{c}\text { Distance } \\
(\mathrm{km})\end{array}$ & $\begin{array}{c}\text { of } \\
\text { cores }\end{array}$ & $\begin{array}{c}P_{t h} \\
(\mathrm{GW})\end{array}$ & $\begin{array}{c}\text { Flux } \\
\left(\bar{\nu}_{e} \mathrm{~cm}^{-2} \mathrm{~s}^{-1}\right)\end{array}$ & $\begin{array}{c}\text { Signal } \\
\left(\bar{\nu}_{e} / \mathrm{yr}\right)\end{array}$ \\
\hline Japan & & & & & \\
Kashiwazaki & 160.0 & 7 & 24.6 & $4.25 \times 10^{5}$ & 348.1 \\
Ohi & 179.5 & 4 & 13.7 & $1.88 \times 10^{5}$ & 154.0 \\
Takahama & 190.6 & 4 & 10.2 & $1.24 \times 10^{5}$ & 101.8 \\
Hamaoka & 214.0 & 4 & 10.6 & $1.03 \times 10^{5}$ & 84.1 \\
Tsuruga & 138.6 & 2 & 4.5 & $1.03 \times 10^{5}$ & 84.7 \\
Shiga & 80.6 & 1 & 1.6 & $1.08 \times 10^{5}$ & 88.8 \\
Mihama & 145.4 & 3 & 4.9 & $1.03 \times 10^{5}$ & 84.5 \\
Fukushima-1 & 344.0 & 6 & 14.2 & $5.3 \times 10^{4}$ & 43.5 \\
Fukushima-2 & 344.0 & 4 & 13.2 & $4.9 \times 10^{4}$ & 40.3 \\
Tokai-II & 294.6 & 1 & 3.3 & $1.7 \times 10^{4}$ & 13.7 \\
Shimane & 414.0 & 2 & 3.8 & $9.9 \times 10^{3}$ & 8.1 \\
Onagawa & 430.2 & 2 & 4.8 & $9.8 \times 10^{3}$ & 8.1 \\
Ikata & 561.2 & 3 & 6.0 & $8.4 \times 10^{3}$ & 6.9 \\
Genkai & 755.4 & 4 & 6.7 & $5.3 \times 10^{3}$ & 4.3 \\
Sendai & 824.1 & 2 & 3.3 & $3.5 \times 10^{3}$ & 2.8 \\
Tomari & 783.5 & 2 & 5.3 & $2.4 \times 10^{3}$ & 2.0 \\
South Korea & & & & & \\
Ulchim & $\sim 750$ & 4 & 11.2 & $8.8 \times 10^{3}$ & 7.2 \\
Wolsong & $\sim 690$ & 4 & 8.1 & $7.5 \times 10^{3}$ & 5.2 \\
Yonggwang & $\sim 940$ & 6 & 16.8 & $8.4 \times 10^{3}$ & 6.9 \\
Kori & $\sim 700$ & 4 & 8.9 & $8.0 \times 10^{3}$ & 6.6 \\
Total & & 69 & 175.7 & $1.34 \times 10^{6}$ & 1101.6 \\
\hline \hline
\end{tabular}

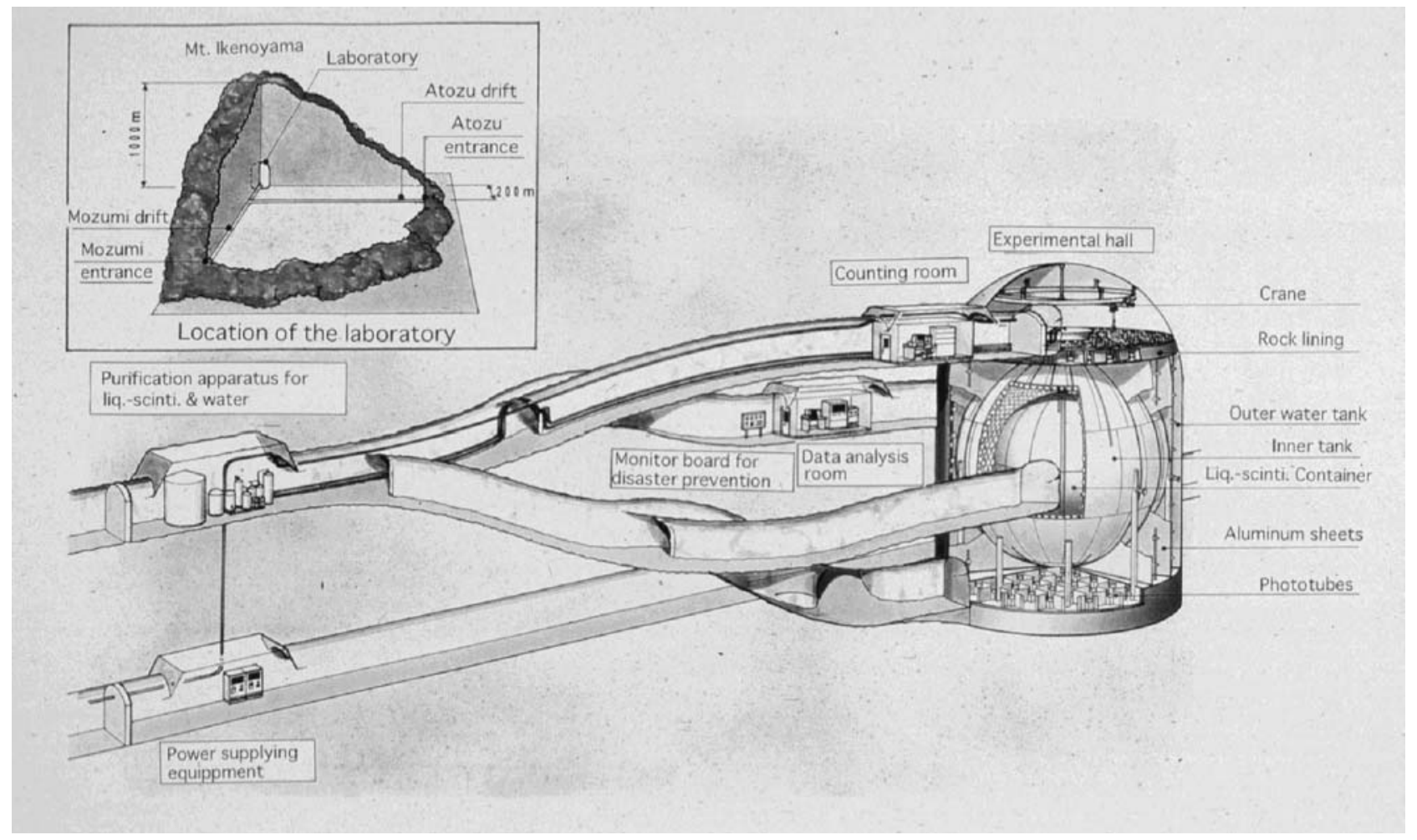

FIG. 33. Partial view of the system of tunnels inside Mount Ikenoyama with the locations of KAMLAND and its main services. 


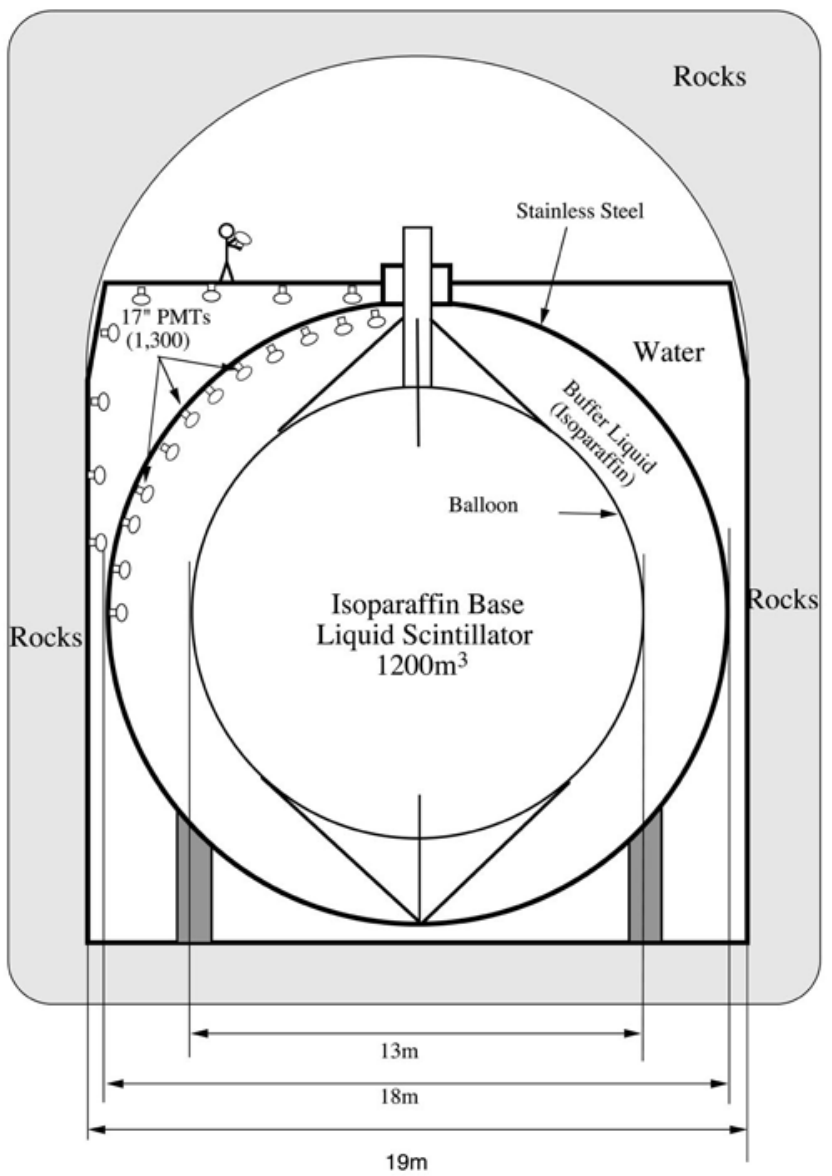

FIG. 34. Schematic cross section of the KAMLAND detector.

to enable the detection of single energy deposits. Of particular importance is the ${ }^{7} \mathrm{Be}$ line, which is below the threshold of water Cerenkov detectors. The presence of

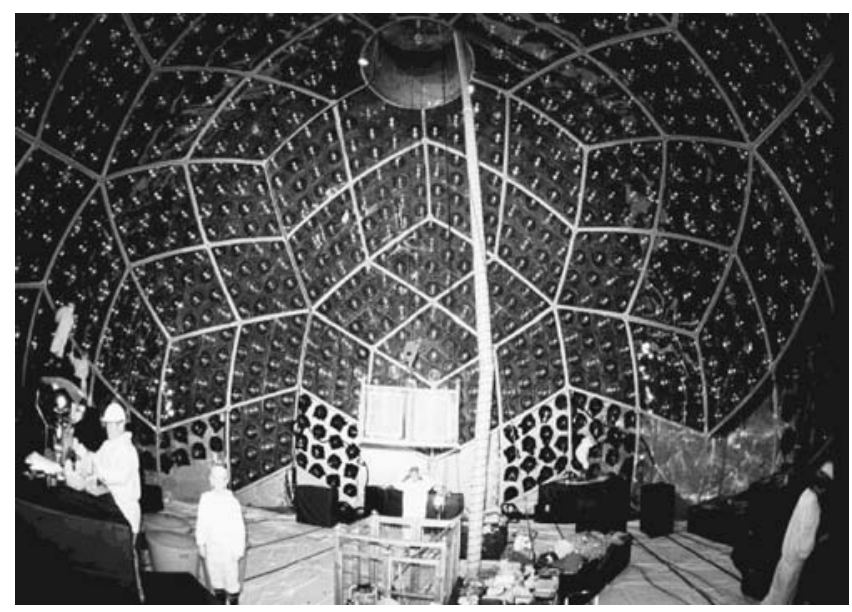

FIG. 35. View of the internal volume of the KAMLAND sphere during the central detector installation. A modular styrofoam raft is used as a platform for workers. The installation began from the top of the sphere and moved down as the water level in the sphere was reduced. PMT's, black shades, acrylic plates, monitoring LED's, and cables were mounted in place for each level before lowering the water.
TABLE VII. Summary of background rates in KAMLAND for the $\bar{\nu}_{e}$ signature. A signal-to-noise ratio of about $10 / 1$ is expected for reactor $\bar{\nu}_{e}$. Adapted from Alivisatos et al. (1998).

\begin{tabular}{lc}
\hline \hline Background source & Rate $\left(\right.$ day $\left.^{-1}\right)$ \\
\hline Cosmic-muon-induced neutrons & 0.1 \\
Natural radioactivity (random coincidence) & 0.15 \\
Natural radioactivity (correlated) & 0.005 \\
Total predicted background & 0.25 \\
Reactor $\bar{\nu}_{e}$ signal (no oscillation) & 2 \\
\hline \hline
\end{tabular}

large amounts of carbon in KAMLAND's scintillator offers the possibility of detailed flavor studies in neutrinos coming from supernovae. Finally, KAMLAND represents such a large step up in size and background relative to the previous detectors that one should be ready for the possibility that it will discover completely new and unexpected phenomena in physics or astrophysics.

Here we shall only mention the topic of terrestrial $\bar{\nu}_{e}$ 's, which is somewhat unusual and directly relates to the experiment's ability to detect $\bar{\nu}_{e}$ 's. The reader interested in the direct detection of solar neutrinos or neutrinos from supernovae is referred to the KAMLAND design report (Alivisatos et al., 1998). A description of the new and unexpected phenomena mentioned above will hopefully be provided at a later stage.

Although the study of terrestrial antineutrinos was proposed as early as 1966 (Eders, 1966), practical difficulties, due to the very small cross sections and very low energies involved, have made their observation impractical until now. KAMLAND has the ability to detect energy depositions of the order of $1 \mathrm{MeV}$ in an unprecedented amount of liquid scintillator and is therefore ideally suited for this study. It is important to realize that low-energy $\bar{\nu}_{e}$ 's are easily detected with very low background in KAMLAND thanks to their very specific signature.

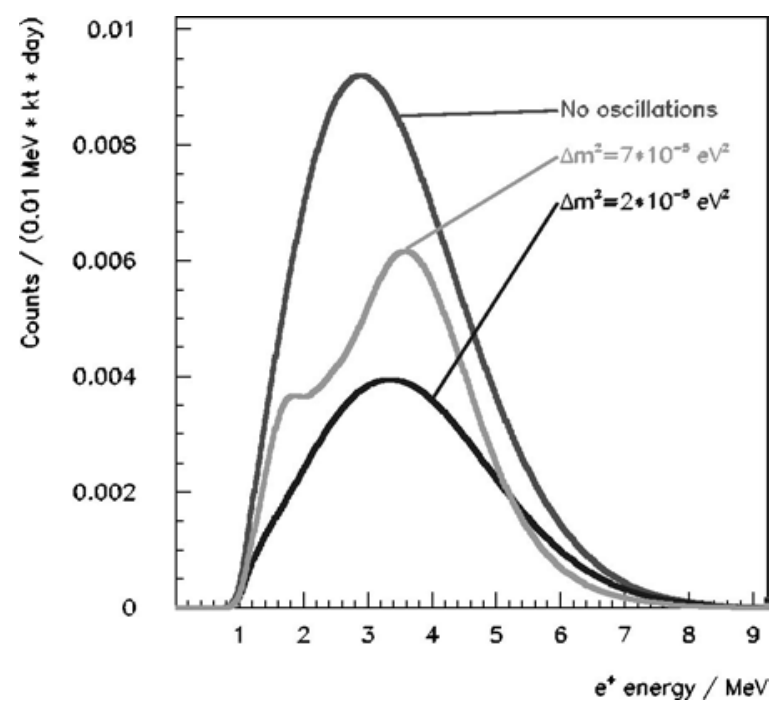

FIG. 36. Positron energy spectra expected at KAMLAND for no oscillations and oscillations with indicated parameters $\Delta m^{2}$ and $\sin ^{2} 2 \theta=0.75$ in the large-mixing-angle MSW solar neutrino solution. 


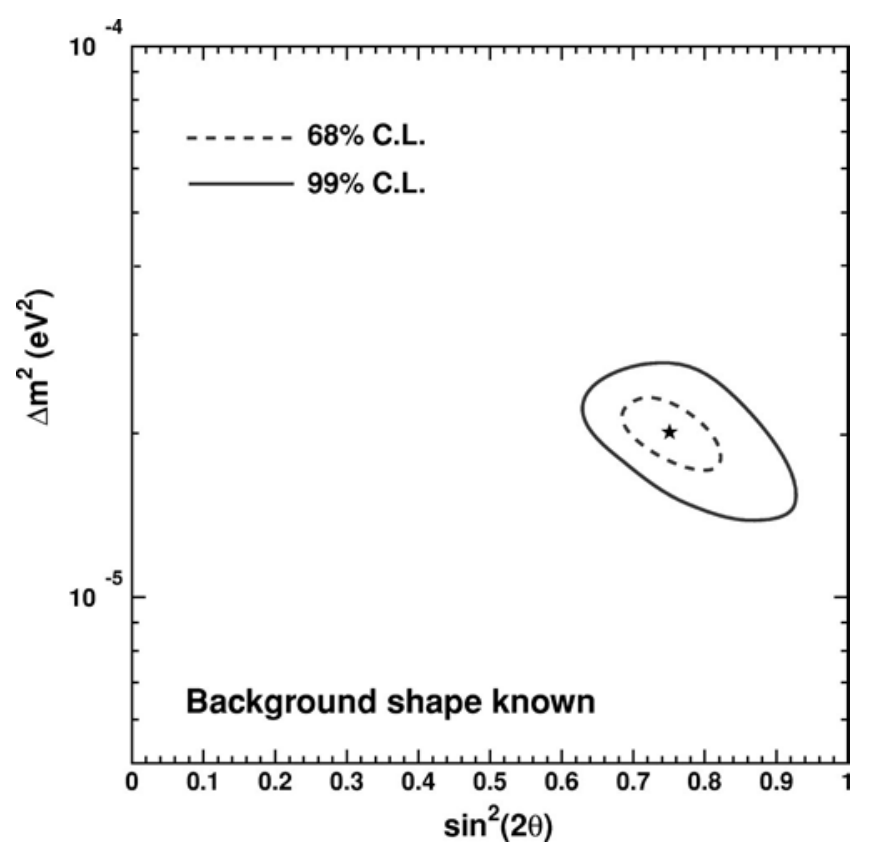

FIG. 37. Simulated measurement of neutrino oscillation parameters from three years of data at KAMLAND assuming $\Delta m^{2}=2 \times 10^{-5} \mathrm{eV}^{2}$ and $\sin ^{2} 2 \theta=0.75$. A signal-to-noise ratio of 10/1 was assumed (see text).

The cooling rate of our planet and its content of heavy elements are central issues in the earth sciences. The earth radiates about $40 \mathrm{TW}$ of heat from its surface. About $40 \%$ of this energy (or $16 \mathrm{TW}$ ) is believed to have radiogenic origin, with $90 \%$ of it deriving from decays of ${ }^{238} \mathrm{U}$ and ${ }^{232} \mathrm{Th}$. Radiogenic heat is therefore an essential component of the present earth dynamics. As discussed by several authors (Eders, 1966; Marx, 1969; Marx and Lux, 1970; Avilez et al., 1981; Krauss et al., 1984), the concentration of these isotopes can be mapped on a planetary scale by direct detection of $\bar{\nu}_{e}$ 's deriving from the $\beta$-decay processes. Since neutrinos have a mean free path many orders of magnitude larger that the size of the earth, the neutrino field is analogous to a gravitational field, where the sources are represented by radioactive density (as opposed to mass density).

Since the maximum energy carried by terrestrial neutrinos is $3.27 \mathrm{MeV}$ (Krauss et al., 1984) and the capture threshold is $1.8 \mathrm{MeV}$, the maximum in the energy spectrum detected in the prompt part of the events will be $2.49 \mathrm{MeV}$ (including the $1.02 \mathrm{MeV}$ from positron annihilations). For energies above threshold only the thorium and uranium decay chains give a detectable number of events. ${ }^{234} \mathrm{~Pa}$ from the $\mathrm{U}$ chain and ${ }^{228} \mathrm{Ac}$ and ${ }^{212} \mathrm{Bi}$ from the $\mathrm{Th}$ chain have similar end points (2.29, 2.08 , and $2.25 \mathrm{MeV}$, respectively), while ${ }^{214} \mathrm{Bi}$ from the $\mathrm{U}$ chain has an end point of $3.27 \mathrm{MeV}$. Therefore the energy spectrum observed for the prompt part of the event should have a characteristic double-hump structure shown in Fig. 38. This will also allow the measurement of the U/Th ratio. Antineutrinos from nuclear reactors give a similar signature, as described above, but their energy is substantially higher and, as shown in the figure,

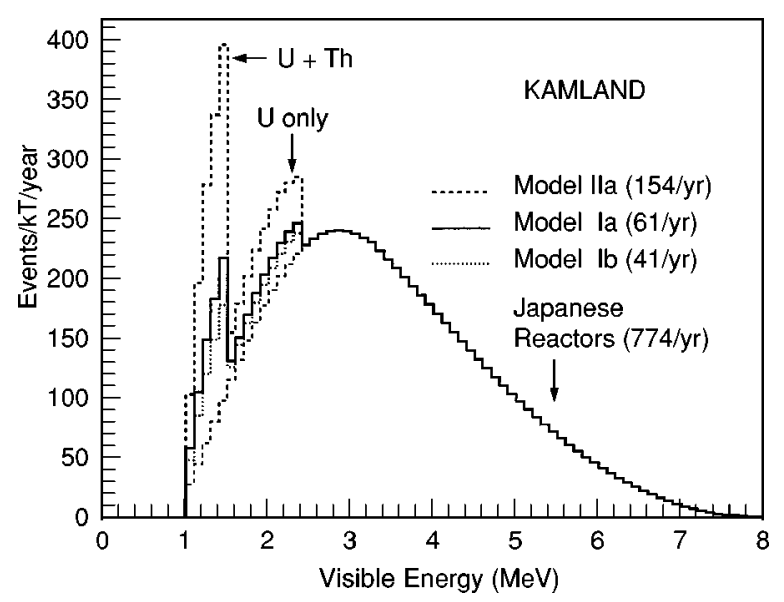

FIG. 38. Energy spectrum from terrestrial antineutrinos compared with reactor signal as expected in KAMLAND. Three different geophysical models are shown for the terrestrial antineutrinos, and no oscillations are assumed for all the spectra shown. Adapted from Raghavan et al. (1998).

they can be easily separated from the terrestrial antineutrinos. Indeed, a repetition of the analysis for the reactor neutrinos discussed above using only positron energies above $2.7 \mathrm{MeV}$ gives an oscillation sensitivity very similar to the one presented in Fig. 37.

The two lower spectra (models Ia and Ib) superimposed in Fig. 38 for the terrestrial antineutrino component correspond to two different possible geophysical models with different heavy-element concentrations in the oceanic and continental crusts (Raghavan et al., 1998). The highest curve (model IIa) is given as a reference and shows what the spectrum would be in the extreme case when the entire $40 \mathrm{TW}$ of heat escaping from the earth's interior was generated by the Th and U decay chains.

In one year of data taking, model Ia would give an integral of 61 events, while model Ib would give only 41 events, and a differentiation between the two at the $3 \sigma$ level could be obtained in five years of datataking, taking into account the fluctuations of the background due to the reactor neutrinos.

The BOREXINO solar neutrino detector at Gran Sasso will also be able to detect terrestrial antineutrinos. The projections (Rothschild, 1998; Schoenert, 1999) suggest that in BOREXINO there will be between 9 and 60 events/year for the geophysical models considered above. The terrestrial antineutrinos might give a stronger signal than the far-away European power reactors which are predicted to give only 27 events/year without oscillations in BOREXINO (Schoenert, 1999).

\section{CONCLUSIONS}

The use of nuclear reactors to study neutrino properties has a long and successful history. While the first experiments devoted to oscillation searches were motivated by the generic principle "look where the light is," many of the modern hints of neutrino oscillations point 
to parameters that match very well the capabilities of reactor-based experiments. At the same time, the understanding of the flux and spectrum of $\bar{\nu}_{e}$ 's from power reactors has reached substantial sophistication. The first two "long-baseline" experiments, CHOOZ and PALO VERDE, have amply demonstrated the capability of this new breed of detectors, while providing solid evidence that $\nu_{e}-\nu_{\mu}$ is not the dominant channel for atmospheric neutrino oscillation. KAMLAND, already taking data now, will extend the reach for small mass differences to unprecedented levels. Its size and background will bring reactor-based experiments to a new level, with several new physics opportunities in the essentially backgroundless detection of $\bar{\nu}_{e}$ 's from a number of natural sources.

\section{ACKNOWLEDGMENTS}

We would like to thank our colleagues from $\mathrm{CHOOZ}$, KAMLAND, and PALO VERDE for the many discussions and help received in understanding the material discussed. This work was supported in part by the US Department of Energy and by the Italian Istituto Nationale di Fisica Nucleare (INFN).

\section{REFERENCES}

Achkar, B., 1992, Ph.D. thesis (Institut Laue-Langevin, Grenoble).

Achkar, B., et al., 1995, Nucl. Phys. B 434, 503.

Achkar, B., et al., 1996, Phys. Lett. B 374, 243.

Aguilar, A., et al., Phys. Rev. D 64, 112007.

Ahmad, Q. R., et al., 2001, Phys. Rev. Lett. 87, 071301.

Alfonin, A. I., et al., 1998, JETP 67, 213.

Alimonti, G., et al., 1998, Astropart. Phys. 8, 141.

Alimonti, G., et al., 2000, Nucl. Instrum. Methods Phys. Res. A 440, 360.

Alivisatos, P., et al. 1998, "KAMLAND: a liquid scintillator Anti-Neutrino Detector at the Kamioka site," preprint Stanford-HEP-98-03, Tohoku-RCNS-98-15.

Amsler, C., et al., 1997, Nucl. Instrum. Methods Phys. Res. A 396, 115.

Apollonio, M., et al., 1998, Phys. Lett. B 420, 397.

Apollonio, M., et al., 1999, Phys. Lett. B 466, 415.

Apollonio, M., et al., 2000, Phys. Rev. D 61, 012001.

Armbruster, B., et al., 1998, Phys. Rev. C 57, 3414.

Athanassopoulos, C., et al., 1995, Phys. Rev. Lett. 75, 2650.

Athanassopoulos, C., et al., 1996, Phys. Rev. C 54, 2685.

Athanassopoulos, C., et al., 1998, Phys. Rev. C 58, 2489.

Avilez, C., G. Marx, and B. Fuentes, 1981, Phys. Rev. D 23, 1116.

Bahcall, J. N., 1989, Neutrino Astrophysics (Cambridge University, Cambridge, England).

Baldini, A., et al., 1996, Nucl. Instrum. Methods Phys. Res. A 372, 207.

Baker, S., and R. Cousins 1984, Nucl. Instrum. Methods Phys. Res. A 221, 437.

Barger, V., K. Whisnant, and R. J. N. Phillips, 1980, Phys. Rev. Lett. 45, 2084.

Beacom, J. F., and P. Vogel, 1999, Phys. Rev. Lett. 83, 5222.
Boehm, F., 2001, in Current Aspects of Neutrino Physics, edited by D. Caldwell (Springer, Berlin), p. 131.

Boehm, F., et al., 2000a, Phys. Rev. Lett. 84, 3764.

Boehm, F., et al., 2000b, Phys. Rev. D 62, 072002.

Boehm, F., et al., 2000c, Phys. Rev. D 62, 092005.

Boehm, F., et al., 2001, Phys. Rev. D 64, 112001.

Boehm, F., and P. Vogel, 1992, Physics of Massive Neutrinos (Cambridge University, Cambridge, England).

Bouchez, J., B. Pichard, J. P. Soirat, M. Spiro, and Y. Declais, 1988, Phys. Lett. B 207, 217.

Cabibbo, N., 1978, Phys. Lett. 72B, 333.

Danby, G., J.-M. Gaillard, K. Goulianos, L. M. Lederman, N. Mistry, M. Schwatz, and J. Steinberger, 1962, Phys. Rev. Lett. 9, 36 .

Davis, B. R., P. Vogel, F. M. Mann, and R. E. Schenter, 1979, Phys. Rev. C 19, 2259.

Declais, Y., et al., 1994, Phys. Lett. B 338, 383.

Derbin, A. I., A. V. Chernyi, L. A. Popeko, V. N. Muratova, G. A. Shishkina, and S. I. Bakhlanov, 1993, JETP Lett. 57, 768. Detwiler, J., 2000, KAMLAND note 00-06, unpublished.

Eadie, W. T., et al., 1971, Statistical Methods of Experimental Physics (North-Holland, Amsterdam).

Eders, G., 1966, Nucl. Phys. 78, 657.

Eitel, K., 2000, Nucl. Phys. B, Proc. Suppl. 91, 191.

Fayans, S. A., 1985, Sov. J. Nucl. Phys. 42, 590.

Feldman, G. J., and R. D. Cousins, 1998, Phys. Rev. D 57, 3873.

Fisher, P., B. Kayser, and K. S. McFarland, 1999, Annu. Rev. Nucl. Part. Sci. 49, 481.

Fukuda, S., et al., 2000, Phys. Rev. Lett. 85, 3999.

Fukuda, S., et al., 2001a, Phys. Rev. Lett. 86, 5651.

Fukuda, S., et al., 2001b, Phys. Rev. Lett. 86, 5656.

Fukuda, Y., et al., 1998, Phys. Rev. Lett. 81, 1562.

Fukuda, Y., et al., 1999, Phys. Rev. Lett. 82, 2430.

Gratta, G., et al., 1997, Nucl. Instrum. Methods Phys. Res. A 400, 456.

Groom, D. E., et al., 2000, Eur. Phys. J. C 15, 1.

Hagner, C., M. Altmann, F. v. Feilitzsch, L. Oberauer, Y. Declais, and E. Kajfasz, 1995, Phys. Rev. D 52, 1343.

Hahn, A. A., K. Schreckenbach, W. Gelletly, F. v. Feiltizsch, G. Colvin, and B. Krusche, 1989, Phys. Lett. B 218, 365.

Harrison, P. F., D. H. Perkins, and W. C. Scott, 1996, Phys. Lett. B 349, 137.

James, F., 1994, MINUIT Reference Manual, vers. 94.1.

Kajita, T., and Y. Totsuka, 2001, Rev. Mod. Phys. 73, 85.

Kirsten, T. A., 2000, Nucl. Phys. B, Proc. Suppl. 87, 152.

Klapdor, H.-V., and J. Metzinger, 1982a, Phys. Rev. Lett. 48, 127.

Klapdor, H.-V., and J. Metzinger, 1982b, Phys. Lett. 112B, 22. Kodama, K., et al., 2001, Phys. Lett. B 504, 218.

Kopeikin, V. I., L. A. Mikaelyan, and V. V. Sinev, 1997, Phys. At. Nucl. 60, 172.

Kozlov, Y. V., S. V. Khaltourtcev, I. N. Machulin, A. V. Martemyanov, V. P. Martemyanov, S. V. Sukhotin, V. G. Tarasenkov, E. V. Turbin, and V. N. Vyrodov, 2000, Phys. At. Nucl. 63, 1016.

Krauss, L., et al., 1984, Nature (London) 310, 191.

Kuvshinnikov, A. A., L. A. Mikaelyan, S. V. Nikolaev, M. D. Skorokhvatov, and A. V. Etenko, 1990, Sov. J. Nucl. Phys. 52, 300.

Kwon, H., F. Boehm, A. A. Hahn, H. E. Henrikson, J.-L. Vuilleumier, J.-F. Cavaignac, D. H. Koang, B. Vignon, F. v. Feilitzsch, and R. L. Mossbauer, 1981, Phys. Rev. D 24, 1097. 
Llewellyn-Smith, C. H., 1972, Phys. Rep. 3, 261.

Maki, Z., M. Nakagawa, and S. Sakata, 1962, Prog. Theor. Phys. 28, 870 .

Marx, G., 1969, Czech. J. Phys., Sect. B 19, 1471.

Marx, G., and I. Lux, 1970, Acta Phys. Acad. Sci. Hung. 28, 63.

Mikaelyan, L., 2000, Nucl. Phys. B, Proc. Suppl. 91, 120.

Mikheyev, S. P., and A. Yu. Smirnov, 1986a, Sov. J. Nucl. Phys. 42, 913.

Mikheyev, S. P., and A. Yu. Smirnov, 1986b, Nuovo Cimento Soc. Ital. Fis., C 91, 17.

Miller, L., 2000, Ph.D. thesis (Stanford University).

Nicolò, D., 1999, Ph.D. thesis (Scuola Normale Superiore, Pisa).

Pasierb, E., H. S. Gurr, J. Lathrop, F. Reines, and H. W. Sobel, 1979, Phys. Rev. Lett. 43, 96.

Perl, M. L., et al., 1975, Phys. Rev. Lett. 35, 1489.

Piepke, A., S. W. Moser, and V. M. Novikov, 1999, Nucl. Instrum. Methods Phys. Res. A 432, 392.

Pontecorvo, B., 1958, Sov. Phys. JETP 6, 429.

Pontecorvo, B., 1967, Sov. Phys. JETP 33, 549.

Oberauer, L., and F. von Feilitzsch, 1987, Phys. Lett. B 198, 113.

Okamura, K., 1999, Ph.D. thesis (University of Tokyo).

Raffelt, G., 1995, Stars as Laboratories for Fundamental Physics (University of Chicago, Chicago).

Raghavan, R. S., S. Schoenert, S. Enamoto, J. Shirai, F. Suekane, and A. Suzuki, 1998, Phys. Rev. Lett. 80, 635.

Reines, F., and C. L. Cowan, Jr., 1953, Phys. Rev. 92, 830.

Reines, F., and C. L. Cowan, Jr., 1959, Phys. Rev. 113, 273.

Reines, F., H. S. Gurr, and H. W. Sobel, 1976, Phys. Rev. Lett. 37, 315 .

Reines, F., H. W. Sobel, and H. S. Gurr, 1974, Phys. Rev. Lett. 32, 180.

Reines, F., H. W. Sobel, and E. Pasierb, 1980, Phys. Rev. Lett. 45, 1307.

Riley, S. P., Z. D. Greenwood, W. R. Kropp, L. R. Price, F.
Reines, H. W. Sobel, Y. Declais, A. Etenko, and M. Skorokhvastov, 1999, Phys. Rev. C 59, 1780.

Rothschild, C. G., M. C. Chen, and F. P. Calaprice, 1998, Geophys. Res. Lett. 25, 1083.

Schoenert, S., 1999, Nucl. Phys. B, Proc. Suppl. 70, 195.

Schoenert, S., L. Oberauer, C. Hagner, F. v. Feilitzsch, K. Schreckenbach, Y. Declais, and Y. Mayerhofer, 1996, Nucl. Phys. B, Proc. Suppl. 48, 201.

Schreckenbach, K., G. Colvin, W. Gelletly, and F. v. Feiltizsch, 1985, Phys. Lett. 160B, 325.

Tengblad, O., K. Aleklett, R. von Dincklage, E. Lund, G. Nyman, and G. Rudstam, 1989, Nucl. Phys. A 503, 136.

Vidyakin, G. S., et al., 1994, JETP 59, 390.

Vogel, P., 1984a, Phys. Rev. D 29, 1918.

Vogel, P., 1984b, Phys. Rev. D 30, 1505.

Vogel, P., and J. F. Beacom, 1999, Phys. Rev. D 60, 053003.

Vogel, P., and J. Engel, 1989, Phys. Rev. D 39, 3378.

Vogel, P., G. K. Schenter, F. M. Mann, and R. E. Schenter, 1981, Phys. Rev. C 24, 1543.

Wang, Y.-F., V. Balic, G. Gratta, A. Fasso, S. Roessler, and A. Ferrari, 2001, Phys. Rev. D 64, 013012.

Wang, Y.-F., L. Miller, and G. Gratta, 2000, Phys. Rev. D 62, 013012.

Wilkinson, D. H., 1982, Nucl. Phys. A 377, 474.

Winter, K., 1991, Ed., Neutrino Physics (Cambridge University, Cambridge, England).

Wojcicki, S., 2001a, Nucl. Phys. B, Proc. Suppl. 91, 216.

Wojcicki, S., 2001b, in Proceedings of the 9th International Workshop on Neutrino Telescopes, Venice, edited by M. Baldo-Ceolin (Istituto Nazionale di Física Nucleare, Padova, Italy).

Wolfenstein, L., 1979, Phys. Rev. D 17, 2369.

Wolfenstein, L., 1980, Phys. Rev. D 20, 2634.

Zacek, G., 1984, Ph.D. thesis (Technical University Munich).

Zacek, G., et al., 1986, Phys. Rev. D 34, 2621.

Zuber, K., 1998, Phys. Rep. 305, 296. 